\title{
Large deviations from a macroscopic scaling limit for particle systems with Kac interaction and random potential
}

\author{
Mustapha Mourragui ${ }^{\mathrm{a}, *}$, Enza Orlandi ${ }^{\mathrm{b}}$ \\ a Université de Rouen, LMRS, UMR 6085, Avenue de l'université, B.P. 12, 76801, Saint Etienne du Rouvray, France \\ ${ }^{\mathrm{b}}$ Dipartimento di Matematica, Universitá di Roma Tre, L.go S.Murialdo 1, 00156 Roma, Italy
}

Received 11 May 2005; accepted 15 September 2006

Available online 27 February 2007

\begin{abstract}
We consider a lattice gas in a periodic $d$-dimensional lattice of width $\gamma^{-1}, \gamma>0$, interacting via a Kac's type interaction, with range $\frac{1}{\gamma}$ and strength $\gamma^{d}$, and under the influence of a random one body potential given by independent, bounded, random variables with translational invariant distribution. The system evolves through a conservative dynamics, i.e. particles jump to nearest neighbor empty sites, with rates satisfying detailed balance with respect to the equilibrium measures. In [M. Mourragui, E. Orlandi, E. Saada, Macroscopic evolution of particles systems with random field Kac interactions, Nonlinearity 16 (2003) 2123-2147] it has been shown that rescaling space as $\gamma^{-1}$ and time as $\gamma^{-2}$, in the limit $\gamma \rightarrow 0$, for dimensions $d \geqslant 3$, the macroscopic density profile $\rho$ satisfies, a.s. with respect to the random field, a non-linear integral partial differential equation, having the diffusion matrix determined by the statistical properties of the external random field. Here we show an almost sure (with respect to the random field) large deviations principle for the empirical measures of such a process. The rate function, which depends on the statistical properties of the external random field, is lower semicontinuous and has compact level sets.
\end{abstract}

() 2007 Elsevier Masson SAS. All rights reserved.

\section{Résumé}

On considère un modèle de spins évoluant dans le tore de dimension $d \geqslant 3$, de largeur $\gamma^{-1}(\gamma>0)$, soumis à un potentiel d'interaction de Kac de portée $\gamma^{-1}$ et à un champ extérieur aléatoire. Le champ extérieur aléatoire est défini par des variables aléatoires indépendantes, bornées dont la loi est supposée invariante par translation. L'évolution du système au cours du temps consiste à échanger l'occupation entre deux sites voisins selon des taux vérifiant la condition du bilan détaillé. La limite hydrodynamique a été étudiée en dimension $d \geqslant 3$ dans [M. Mourragui, E. Orlandi, E. Saada, Macroscopic evolution of particles systems with random field Kac interactions, Nonlinearity 16 (2003) 2123-2147]. Les auteurs ont démontré que sous l'échelle spatiale $\gamma^{-1}$ et l'échelle temporelle $\gamma^{-2}$, pour presque tout environnement aléatoire, les mesures empiriques convergent vers l'unique solution faible d'une équation de second ordre définie à partir d'une matrice de diffusion. Dans ce papier nous établissons pour presque tout environnement aléatoire, un principe de grandes déviations pour ce modèle. La fonctionnelle d'action associée aux grandes déviations est semi-continue inférieurement et admet des ensembles de niveaux compacts.

(c) 2007 Elsevier Masson SAS. All rights reserved.

MSC: 60K35; 82C22; 60F10

\footnotetext{
Work supported by MURST/Cofin 05-06 PRIN 2004028108, GDRE 224, CNRS-INDAM University of Rouen.

* Corresponding author.

E-mail addresses: Mustapha.Mourragui@univ-rouen.fr (M. Mourragui), orlandi@mat.uniroma3.it (E. Orlandi).
} 


\section{Introduction}

Models where a stochastic contribution is added to the energy of the system naturally arise in condensed matter physics where the presence of the impurities causes the microscopic structure to vary from point to point. An extensive literature has been dedicated to study the equilibrium statistical properties of (spin) systems with external random field. The central question heatedly discussed in the 1980's in the physics community was whether the Random Field Ising model would show spontaneous magnetization at low temperature and weak disorder in dimension 3, or not. The problem was solved by Bricmont and Kupianen, [4], who proved the existence of phase transition in $d \geqslant 3$ for small magnitude of the random field, and Aizenman and Wehr, [1], who proved that there is no phase transition in $d=2$ for all temperatures. A more physical oriented review about Random Field Ising model is [22].

The Kac's potentials are two body interactions with range $\frac{1}{\gamma}$ and strength $\gamma^{d}$, where $\gamma>0$ is a dimensionless parameter which represents the ratio between microscopic and macroscopic lengths. When $\gamma \rightarrow 0$, i.e. very long range compared with the interparticle spacing, the strength of the interaction becomes very weak, but in such a way that the total interaction between one particle and all the others is kept finite. They were introduced in [12], and then generalized in [18], to present a rigorous derivation of the van der Waals theory of a gas-liquid phase transition. In the last decade many authors studied the equilibrium statistical properties of systems with Kac potential for $\gamma$ small but finite and the time evolution of the macroscopic density profile in particle systems interacting via long range Kac potential either in the case of conservative dynamics [17,9,10,20], or in the case of non-conservative dynamics [7]. For a review of various results concerning these models, see [11,23,3]. Random Field Kac models, in $d=1$ and for $\gamma$ small and fixed, have been recently studied in [5,6]. The particle model studied in [21] and here is a dynamic version of lattice gases interacting via a two-body Kac interaction and subject to external random field given by independent bounded random variables with translational invariant distribution. The formal Hamiltonian we consider is given by

$$
H_{\gamma}^{\beta, \alpha}(\eta)=-\frac{\beta}{2} \sum_{x, y \in \mathbb{Z}^{d}} J_{\gamma}(x-y) \eta(x) \eta(y)-\sum_{x \in \mathbb{Z}^{d}} \alpha(x) \eta(x),
$$

where $\beta$ is a positive parameter and $\eta(x) \in\{0,1\}, \eta(x)=1$ if there is a particle in $x$ and $\eta(x)=0$ means that the site is empty. The $\left\{\alpha(x), x \in \mathbb{Z}^{d}\right\}$ represents the external random field on the sites $x$. Given the Hamiltonian (1.1) there is a standard way, see for example $[28,16]$, to construct a dynamic which conserves the number of particles and for which the invariant measures are given by the one parameter family of Gibbs measures associated to (1.1). More precise statements will be given in Section 2. The relevant features of the systems associated to (1.1) are the absence of translation invariance, for a given disorder configuration, and the non-validity of the so called gradient condition. To establish the hydrodynamic limit one needs to show some version of Fick's law, namely to replace the microscopic current (i.e. the difference between the rate at which a particle jumps from site $x$ to site $y$ and the rate at which a particle jumps from site $y$ to site $x, x$ and $y$ being nearest neighbors) by the gradient of the density field multiplied by the diffusion coefficient. Roughly speaking, the gradient condition says that the microscopic current is already the gradient of a function of the density field. Performing a diffusive scaling limit, in [21], for almost all disorder, a law of large numbers when $d \geqslant 3$ was established for the density field, starting from a sequence of measures associated to some initial density profile $\rho_{0}, 0 \leqslant \rho_{0} \leqslant 1$. The equation obtained for the density field is the following non-local, non-linear partial differential equation

$$
\frac{\partial \rho}{\partial t}=\frac{1}{2} \nabla \cdot\left(\sigma(\rho) \nabla \frac{\delta \mathcal{G}}{\delta \rho}\right), \quad \rho(0, r)=\rho_{0}(r),
$$

where the energy functional $\mathcal{G}(\rho)$ is a suitable non-linear integral functional, see $(2.27)$ and $\frac{1}{2} \sigma(\rho)$ is the mobility, see (2.22), ${ }^{1}$ or conductivity, of the system with only short range interaction, i.e. corresponding to $\beta \equiv 0$ in (1.1).

\footnotetext{
1 In the physical literature one writes the mobility as $\sigma_{1}(\rho)=\frac{1}{2} \sigma(\rho)$. We assumed this convection in [21]. So the $\sigma(\rho)$ in [21] does correspond to $\frac{1}{2}$ of the quantity denoted here with the same symbol.
} 
Faggionato and Martinelli, [8], proved for the process associated to (1.1) with $\beta=0$, in the diffusive scaling limit the almost sure existence of the hydrodynamical limit. The result in [21] is obtained applying "a perturbation argument" based on their result. Since the original particle model cannot have more than one particle at a lattice site, $\sigma(0)=$ $\sigma(1)=0$, the solution $\rho$ of (1.2) is bounded between 0 and 1 . The control on the regularity of the mobility $\sigma(\cdot)$ is harder, so far only continuity has been proven, see [8], even though from physical arguments one expects more regularity, see [13], page 179. In the following we will assume, when needed, more regularity for $\sigma(\rho)$. The main interest in studying models with Kac's type of interaction and local interaction, relies on the fact that one can derive the macroscopic equation for the conserved quantity even if the full system undergoes to phase transition, provide the local interaction which in the case at handle is given by the one body random interaction (i.e obtaining setting $\beta=0$ in (1.1)), does not undergo to a phase transition. In this regime the equilibrium statistical properties of the full Hamiltonian do not matter. For more details on this issue we refer to [10].

We are interested in proving large deviations principles for the empirical random density of the process just described. For $\gamma>0$ and for a realization of the random field let $Q_{\gamma}^{\text {noneq }}$ be the process corresponding to the randomly evolving empirical density starting from some initial non-equilibrium state. The law of the large number derived in [21] tell us that for any reasonable nice set $\mathcal{A}$ of measure valued trajectories not containing $\rho(t, r) \mathrm{d} r$, where $\rho(t, \cdot)$ is the solution of the non-linear macroscopic equation (1.2), almost surely with respect to the disorder, $Q_{\gamma}^{\text {noneq }}[A] \rightarrow 0$ as $\gamma \rightarrow 0$. In this paper we can say how fast:

$$
Q_{\gamma}^{\text {noneq }}\{\mathcal{A}\} \sim \exp \left\{-\gamma^{-d} \inf _{\mu(\cdot, \cdot) \in \mathcal{A}} \mathcal{I}_{T}(\{\mu\})\right\},
$$

where $\mathcal{I}_{T}$ is a suitable non-negative functional depending on the all process up to the time $T$ and on the statistical properties of the random field. The $\sim$ denotes logarithmic equivalence as $\gamma \rightarrow 0$. As it is well known, the rate functional $\mathcal{I}_{T}(\cdot)$ is determined by two distinct types of large deviations of the same order. The first one corresponds to large deviations from the initial state and it is quite simple to obtain. The second one derives from the stochastic character of the evolution. Suppose $\mathcal{A}=\left\{\pi_{\gamma}(\cdot, \mathrm{d} r) \simeq m(\cdot, r) \mathrm{d} r, t \in[0, T]\right\}$ where $\pi_{\gamma}(\cdot, \mathrm{d} r)$ is the local density, $\simeq$ denotes closeness in some norm and $m$ is a profile different from the solution $\rho$ of the non-linear macroscopic equation (1.2). We need to modify $Q_{\gamma}^{\text {noneq }}$ so that the event $\mathcal{A}$ becomes typical. One possible choice is to drive the lattice gas by weak, slowly varying, space-time dependent external forces in such a way that the path measure $t \in[0, T] \rightarrow m(t, \cdot)$ becomes typical. This is the standard choice in the case of gradient systems. For non-gradient systems the force must be configuration dependent (see [28], page 248) to take in account that for these systems the response in the current to an external force field is partially delayed. Since we have an external random field, it might be random depending as well. A priori is not clear trough which mechanism the event under consideration should be made typical. Following Donsker and Varadhan to prove the upper bound for (1.3), we construct a family of mean 1 positive $Q_{\gamma}^{\text {noneq }}-$ martingales that can be expressed as function of the empirical measures. The relevant positive martingales are obtained as small Markovian perturbations of the original process, i.e adding to the original process a slowly varying, space-time dependent external forces depending on the configuration and on the randomness. The scheme of our proof goes along the same pattern of [25], where large deviations for a non-gradient version of Ginzburg-Landau model were proved and [26] where large deviations for the symmetric simple exclusion process in dimensions $d \geqslant 3$ have been shown.

The proof of the lower bound relies on proving a law of large numbers for the empirical measures evolving according to a process obtained adding, as explained before, a weak driving force into the original system which depends on local configuration and on the randomness. The choice done is suggested by the variational formula for the diffusion matrix obtained in [8]. The random part can be felt only at microscopic level and it does not change the macroscopic limit. It is needed to reconstruct in the lower bound the microscopic part needed in the variational form of the mobility. Then one shows that upper bound and lower bound coincide. The final step is to prove that for an open set of paths $\mathcal{A}$ the $\inf _{\mu(\cdot, \cdot) \in \mathcal{A}} \mathcal{I}_{T}(\{\mu\})$ does not change if the infimum is taken only over a convenient subset of $\mathcal{A}$. One difficulty in showing upper and lower bound is that the rate functional, see (2.32), might not be convex as function of $\mu$, so lower semicontinuity and extension of the lower bound are far from trivial. Results in all dimensions for a process associated to the Hamiltonian (1.1) with $\beta=0$, were announced by Quastel in [24]. In [2] an exclusion process interacting with ferromagnetic, (i.e $J \geqslant 0$ ), Kac potential was studied and as an intermediate result, large deviations for the empirical measures of the process were shown. The proof relies strongly on the large deviations result for the symmetric simple exclusion process, see [15].

Outline. In Section 2 we state notations, model and results. In Section 3 we prove the lower semicontinuity of the rate function and the compactness of its level sets. Since the methods to establish the upper and lower bounds are 
fairly close to the ones providing law of large numbers we start recalling in Section 4 the steps to prove the law of large numbers for a system where space-time dependent external forces depending on the configuration and on the randomness have been added. This is in the same line of the law of large numbers proven in [21] even though the interaction we consider here is slightly more general, for the reasons explained before. In Section 5 we prove the upper bound. Then in Section 6 we show the lower bound, carrying out in Section 7 its extension. We recall some properties of non-gradient systems, needed along the way, in Section 8.

\section{The model and the main results}

\subsection{The model}

We consider the $d$-dimensional lattice $\mathbb{Z}^{d}$ with sites $x=\left(x_{1}, \ldots, x_{d}\right)$ and canonical basis $\mathcal{E}=\left\{e_{1}, \ldots, e_{d}\right\}$. We denote by $\Lambda$ the $d$-dimensional torus of diameter 1 . Let $\gamma \in(0,1)$ be the scaling parameter so that $\gamma^{-1} \in \mathbb{N}$. We denote by $\Lambda_{\gamma} \equiv \mathbb{Z}^{d} / \gamma^{-1} \mathbb{Z}^{d}$ the discrete torus of diameter $\gamma^{-1}$ and by $\left|\Lambda_{\gamma}\right|$ its cardinality. Same convention will be used to denote the cardinality of any finite non-empty subset of $\mathbb{Z}^{d}$.

For a fixed $A>0$, let $\Omega_{D}=[-A, A]^{\mathbb{Z}^{d}}$ be the set of disorder configurations on $\mathbb{Z}^{d}$. On $\Omega_{D}$ we define a product, translation invariant probability measure $\mathbb{P}$. We denote by $\mathbb{E}$ the expectation with respect to $\mathbb{P}$, and by $\alpha \equiv\{\alpha(x), x \in$ $\left.\mathbb{Z}^{d}\right\}, \alpha(x) \in[-A, A]$, a disorder configuration in $\Omega_{D}$. A configuration $\alpha \in \Omega_{D}$ induces in a natural way a disorder configuration $\alpha_{\gamma}$ on $\Lambda_{\gamma}$, by identifying a cube centered at the origin of side $\gamma^{-1}$ ( $\gamma^{-1}$ odd and integer) with the torus $\Lambda_{\gamma}$. By a slight abuse of notation whenever in the following we refer to a disorder configuration either on $\Lambda_{\gamma}$ or on $\mathbb{Z}^{d}$ we denote it by $\alpha$. We denote by $\mathcal{S}_{\gamma} \equiv\{0,+1\}^{\Lambda_{\gamma}}$ and $\mathcal{S} \equiv\{0,+1\}^{\mathbb{Z}^{d}}$ the configuration spaces, both equipped with the product topology. We denote by $\eta$ a configuration, either in $\mathcal{S}_{\gamma}$ or in $\mathcal{S}$. Given $\alpha \in \Omega_{D}$ and $\beta$ a positive parameter, we consider the Hamiltonian (1.1) restricted to the torus $\Lambda_{\gamma}$, and write it as the sum of two terms

$$
H_{\gamma}(\eta)=\beta H_{\gamma}^{K}(\eta)+H_{0}^{\alpha}(\eta), \quad \eta \in \mathcal{S}_{\gamma},
$$

where $H_{0}^{\alpha}$ is the local, one body, random interaction,

$$
H_{0}^{\alpha}(\eta)=-\sum_{x \in \Lambda_{\gamma}} \alpha(x) \eta(x)
$$

and $H_{\gamma}^{K}$ is the long range Kac interaction,

$$
H_{\gamma}^{K}(\eta)=-\frac{1}{2} \sum_{(x, y) \in \Lambda_{\gamma} \times \Lambda_{\gamma}} J_{\gamma}(x-y) \eta(x) \eta(y) .
$$

The pair interaction $J_{\gamma}(x-y)$, the so-called Kac potential, is such that $J_{\gamma}(x-y) \equiv \gamma^{d} J(\gamma(x-y))$ for $J \in \mathcal{C}^{2}(\Lambda, \mathbb{R})$ with $J(r)=J(-r)$ (symmetry). We denote by $\mu_{\gamma}^{\beta, \alpha, \lambda}$ the grand canonical random Gibbs measure on $\mathcal{S}_{\gamma}$ associated to the Hamiltonian (2.1) with chemical potential $\lambda \in \mathbb{R}$

$$
\mu_{\gamma}^{\beta, \alpha, \lambda}(\eta)=\frac{1}{Z_{\gamma}^{\beta, \alpha, \lambda}} \exp \left\{-H_{\gamma}(\eta)+\lambda \sum_{x \in \Lambda_{\gamma}} \eta(x)\right\}, \quad \eta \in \mathcal{S}_{\gamma},
$$

where $Z_{\gamma}^{\beta, \alpha, \lambda}$ is the normalization factor, so that $\mu_{\gamma}^{\beta, \alpha, \lambda}$ is a probability measure on $\mathcal{S}_{\gamma}$. When $\beta=0, \mu_{\gamma}^{0, \alpha, \lambda}$ becomes the random Bernoulli product measure

$$
\mu_{\gamma}^{\alpha, \lambda}(\eta) \equiv \mu_{\gamma}^{0, \alpha, \lambda}(\eta)=\frac{\prod_{x \in \Lambda_{\gamma}} \mathrm{e}^{[\alpha(x)+\lambda] \eta(x)}}{\prod_{x \in \Lambda_{\gamma}}\left[\mathrm{e}^{[\alpha(x)+\lambda]}+1\right]}, \quad \eta \in \mathcal{S}_{\gamma} .
$$

If $\lambda=0$, we simply write $\mu_{\gamma}^{\alpha}$. We denote by $\mu^{\alpha, \lambda}(\eta)$ and when $\lambda=0, \mu^{\alpha}$ the measure (2.5) on the infinite product space $\mathcal{S}$. Moreover, for a probability measure $\mu$ and a bounded function $f$, both defined on $\mathcal{S}$ or $\mathcal{S}_{\gamma}$, we denote by $\mathbf{E}^{\mu}(f)$ the expectation of $f$ with respect to $\mu$. As it is well known, the chemical potential $\lambda$ is canonically conjugate to the density $\rho$ in the sense that the average density with respect to $\mu_{\gamma}^{\beta, \alpha, \lambda}$ is equal to $\rho$. So as in [8] one can 
define the random empirical chemical potential and the annealed chemical potential $\lambda_{0}(\rho)$. To our aim it is enough to consider $\lambda_{0}(\rho)$. For $\rho \in[0,1]$, the function $\lambda_{0}(\rho)$ is defined as the unique $\lambda$ so that

$$
\mathbb{E}\left[\int \eta(0) \mathrm{d} \mu^{\alpha, \lambda}(\eta)\right]=\mathbb{E}\left[\frac{\mathrm{e}^{\alpha(0)+\lambda}}{1+\mathrm{e}^{\alpha(0)+\lambda}}\right]=\rho .
$$

The disordered Kawasaki dynamics is the Markov process on $\mathcal{S}_{\gamma}$ defined through its infinitesimal generator $\mathcal{L}_{\gamma}$, acting on functions $f: \mathcal{S}_{\gamma} \rightarrow \mathbb{R}$ as

$$
\left(\mathcal{L}_{\gamma} f\right)(\eta)=\sum_{e \in \mathcal{E}} \sum_{x \in \Lambda_{\gamma}} C_{\gamma}(x, x+e ; \eta)\left[\left(\nabla_{x, x+e} f\right)(\eta)\right],
$$

where $e$ is a generic element of $\mathcal{E}$. We omit to write in the notation the explicit dependence on the randomness $\alpha$, unless there is an ambiguity. For $x, y \in \Lambda_{\gamma}, \eta \in \mathcal{S}_{\gamma}$,

$$
\left(\nabla_{x, y} f\right)(\eta)=f\left(\eta^{x, y}\right)-f(\eta)
$$

where $\eta^{x, y}$ is the configuration obtained from $\eta$ by interchanging the values at $x$ and $y$ :

$$
\eta^{x, y}(z)= \begin{cases}\eta(x) & \text { if } z=y \\ \eta(y) & \text { if } z=x \\ \eta(z) & \text { otherwise. }\end{cases}
$$

The rate $C_{\gamma}$ is given by

$$
C_{\gamma}(x, y ; \eta) \equiv C_{\gamma}^{\alpha}(x, y ; \eta)=\Phi\left\{\left(\nabla_{x, y} H_{\gamma}\right)(\eta)\right\} .
$$

Here $\Phi \in C^{2}(\mathbb{R},(0, \infty))$ satisfies $\Phi(0)=1$ and the detailed balance condition

$$
\Phi(r)=\exp (-r) \Phi(-r), \quad r \in \mathbb{R} .
$$

Notice that $C_{\gamma}(x, y ; \eta)$ has the following properties:

(a) detailed balance condition, see (2.10),

(b) positivity and boundedness: there exists $a>0$ such that

$$
a^{-1} \leqslant C_{\gamma}(x, y ; \eta) \leqslant a,
$$

(c) translation covariant:

$$
C_{\gamma}^{\alpha}(x, y ; \eta)=C_{\gamma}^{\tau_{z} \alpha}\left(x-z, y-z ; \tau_{z} \eta\right)=\tau_{z} C_{\gamma}^{\alpha}(x-z, y-z ; \eta),
$$

where for $z$ in $\mathbb{Z}^{d}, \tau_{z}$ denotes the space shift by $z$ units on $\mathcal{S} \times \Omega_{D}$ defined for all $\eta \in \mathcal{S}, \alpha \in \Omega_{D}$ and $g: \mathcal{S} \times \Omega_{D} \rightarrow \mathbb{R}$ by

$$
\left(\tau_{z} \eta\right)(x)=\eta(x+z), \quad\left(\tau_{z} \alpha\right)(x)=\alpha(x+z), \quad\left(\tau_{z} g\right)(\eta, \alpha)=g\left(\tau_{z} \eta, \tau_{z} \alpha\right) .
$$

For each $\lambda \in \mathbb{R}$, the generator $\mathcal{L}_{\gamma}$ is self-adjoint in $L^{2}\left(\mu_{\gamma}^{\beta, \alpha, \lambda}\right)$ (cf. (2.4)). We could alternatively have fixed the number of particles, and got a density $\rho \in\left[0, \frac{1}{\left|\Lambda_{\gamma}\right|}, \ldots, 1\right]$. Then the generator $\mathcal{L}_{\gamma}$ is self-adjoint in $L^{2}\left(v_{\rho, \Lambda_{\gamma}}^{\beta, \alpha}\right)$ for the canonical measure

$$
\nu_{\rho, \Lambda_{\gamma}}^{\beta, \alpha}(\eta)=\frac{1}{Z_{\gamma, \rho}^{\beta, \alpha}} \exp \left\{-H_{\gamma}(\eta)\right\} \mathbb{1}_{\left\{\sum_{x \in \Lambda_{\gamma}} \eta(x)=\rho\left|\Lambda_{\gamma}\right|\right\}}, \quad \eta \in \mathcal{S}_{\gamma}
$$

with $Z_{\gamma, \rho}^{\beta, \alpha}$ the corresponding normalization factor.

To prove the results stated next we need an ancillary process, the Markov process having generator $\mathcal{L}_{\gamma}^{0}$ constructed from the Hamiltonian $H_{0}^{\alpha}$, i.e. with $\beta=0$, see (2.2),

$$
\left(\mathcal{L}_{\gamma}^{0} f\right)(\eta)=\sum_{e \in \mathcal{E}} \sum_{x \in \Lambda_{\gamma}} C^{0}(x, x+e ; \eta)\left[\left(\nabla_{x, x+e} f\right)(\eta)\right],
$$


where $f$ is a function on $\mathcal{S}_{\gamma}$, and

$$
C^{0}(x, y ; \eta)=\Phi\left\{\left(\nabla_{x, y} H_{0}^{\alpha}\right)(\eta)\right\} .
$$

The rate $C^{0}(x, y ; \eta)$ satisfies properties (2.10), (2.11) and (2.12). The process with generator $\mathcal{L}_{\gamma}^{0}$ is the one considered in [8], its invariant measures are, for $\lambda \in \mathbb{R}$, the random product measures $\mu_{\gamma}^{\alpha, \lambda}$ defined in (2.5), or alternatively, for $\rho \in[0,1]$, the canonical measures obtained setting $\beta=0$ in (2.14),

$$
v_{\rho, \Lambda_{\gamma}}^{\alpha}(\eta) \equiv v_{\rho, \Lambda_{\gamma}}^{0, \alpha}(\eta), \quad \eta \in \mathcal{S}_{\gamma} .
$$

Same way, the operator $\mathcal{L}_{\gamma}^{0}$ is self-adjoint in $L^{2}\left(\mu_{\gamma}^{\alpha, \lambda}\right)$, or alternatively in $L^{2}\left(v_{\rho, \Lambda_{\gamma}}^{\alpha}\right)$.

\subsection{The macroscopic equation}

One of the first result of [8] concerns the existence and regularity of the diffusion coefficient $D(\rho)$ which corresponds to the usual Green-Kubo matrix, see [28], Proposition 2.2, page 180. In our set up $D(\rho)$ is the diffusion coefficient of the integral parabolic equation. To define it, let ${ }^{2}$

$$
\mathbb{G} \equiv\left\{g: \mathcal{S} \times \Omega_{D} \rightarrow \mathbb{R} ; \text { local and bounded }\right\},
$$

and for $g \in \mathbb{G}, \Gamma_{g}(\eta)=\sum_{x \in \mathbb{Z}^{d}}\left(\tau_{x} g\right)(\eta, \alpha)$. The $\Gamma_{g}(\eta)$ is a formal expression, but the difference $\left(\nabla_{0, e} \Gamma_{g}\right)(\eta)=$ $\Gamma_{g}\left(\eta^{0, e}\right)-\Gamma_{g}(\eta)$ for $e \in \mathcal{E}$ is meaningful. For each $\rho \in(0,1)$, let $D(\rho)=\left\{D_{i, j}(\rho), 1 \leqslant i, j \leqslant d\right\}$ be the symmetric matrix defined, for every $a \in \mathbb{R}^{d}$, by the variational formula

$$
(a \cdot D(\rho) a)=\frac{1}{2 \chi(\rho)} \inf _{g \in \mathbb{G}} \sum_{i=1}^{d} \mathbb{E}\left[\mathbf{E}^{\mu^{\alpha, \lambda_{0}(\rho)}}\left(C^{0}\left(0, e_{i} ; \eta\right)\left\{a_{i} \nabla_{0, e_{i}} \eta(0)+\left(\nabla_{0, e_{i}} \Gamma_{g}\right)(\eta)\right\}^{2}\right)\right],
$$

where $\lambda_{0}(\rho)$ is defined in (2.6), $\chi(\rho)$ is the static compressibility given by

$$
\chi(\rho)=\mathbb{E}\left[\int \eta(0)^{2} \mathrm{~d} \mu^{\alpha, \lambda_{0}(\rho)}(\eta)-\left(\int \eta(0) d \mu^{\alpha, \lambda_{0}(\rho)}(\eta)\right)^{2}\right],
$$

for $a, b \in \mathbb{R}^{d},(a \cdot b)$ is the scalar vector product of $a$ and $b$ and, recall, $\mathbf{E}^{\mu^{\alpha, \lambda} 0_{0}(\rho)}(\cdot)$ is the expectation with respect to $\mu^{\alpha, \lambda_{0}(\rho)}$, see (2.5), the random Bernoulli product measure with annealed chemical potential $\lambda_{0}(\rho)$. In Theorem 2.1 of [8] it has been proved, for $d \geqslant 3$ and for $\rho \in(0,1)$, the existence of the symmetric diffusion matrix defined in (2.19). Further it has been proved that the coefficients $D_{i, j}(\cdot)$ are non-linear continuous functions in the open interval $(0,1)$ and there exists a constant $C>1$, depending on dimensions and bound on the random field, such that

$$
\frac{\mathbb{1}}{C} \leqslant D(\rho) \leqslant C \mathbb{1}, \quad \rho \in(0,1),
$$

where $\mathbb{1}$ is the $d \times d$ identity matrix. One expects the matrix $D(\cdot)$ to be extended continuously to the closed interval $[0,1]$ and actually to be a smooth function of $\rho$, [13]. The diffusion matrix $D(\rho)$ in a solid, in a regime of linear response, is linked to the mobility $\frac{1}{2} \sigma(\rho)$, see [28], via the Einstein relation

$$
D(\rho)=\frac{1}{2} \sigma(\rho) \chi(\rho)^{-1}
$$

The $\chi(\rho)$ is a smooth function of $\rho$ in $[0,1]$ and it can be easily proven from $(2.20)$ that

$$
\frac{1}{2} \rho(1-\rho) \leqslant \chi(\rho) \leqslant \rho(1-\rho) ; \quad \frac{1}{C} \rho(1-\rho) \mathbb{1} \leqslant \sigma(\rho) \leqslant C \mathbb{1} \rho(1-\rho),
$$

where $C$ is a constant that may change from one occurrence to the next. The bound on $\sigma(\cdot)$ is a simple consequence of the bound on $\chi(\cdot),(2.22)$ and (2.21). Fix a positive time $T$. For a measure $\mu_{\gamma}$ on $\mathcal{S}_{\gamma}$, denote by $\mathbf{P}_{\mu_{\gamma}}$ the probability measure on the path space $D\left([0, T], \mathcal{S}_{\gamma}\right)$ corresponding to the Markov process $\left(\eta_{t}\right)_{t \in[0, T]}$ with generator $\gamma^{-2} \mathcal{L}_{\gamma}$

\footnotetext{
${ }^{2}$ A function $g: \mathcal{S} \times \Omega_{D} \rightarrow \mathbb{R}$ is local if the support of $g, \Delta_{g}$, i.e. the smallest subset of $\mathbb{Z}^{d}$ such that $g$ depends only on $\left\{(\eta(x), \alpha(x)) x \in \Delta_{g}\right\}$, is finite. The function $g$ is bounded if $\sup _{\eta} \sup _{\alpha}|g(\eta, \alpha)|<\infty$.
} 
starting from $\mu_{\gamma}$, and by $\mathbf{E}_{\mu_{\gamma}}$ the expectation with respect to $\mathbf{P}_{\mu_{\gamma}}$. For $t \in[0, T], \eta \in \mathcal{S}_{\gamma}$, let the empirical measure $\pi_{t}^{\gamma}$ be defined by

$$
\pi_{t}^{\gamma}(r) \equiv \pi^{\gamma}\left(r ; \eta_{t}\right)=\gamma^{d} \sum_{x \in \Lambda_{\gamma}} \eta_{t}(x) \delta_{\gamma x}(r), \quad r \in \Lambda,
$$

where $\delta_{u}(\cdot)$ is the Dirac measure on $\Lambda$ concentrated on $u$. Since $\eta(x) \in\{0,1\}$, relation (2.24) induces from $\mathbf{P}_{\mu_{\gamma}}$ a distribution $Q_{\mu_{\gamma}}$ of $\left\{\pi_{t}^{\gamma}(r) ; r \in \Lambda ; t \in[0, T]\right\}$ on the Skorohod space $D\left([0, T], \mathcal{M}_{1}(\Lambda)\right)$, where $\mathcal{M}_{1}(\Lambda)$ is the set of positive Borel measures on $\Lambda$ with total mass bounded by 1 , endowed with the weak topology. Denote by $\mathcal{M}_{1}^{0}(\Lambda)$ the subset of $\mathcal{M}_{1}(\Lambda)$ of all absolutely continuous measures w.r.t. the Lebesgue measure with density bounded by 1 :

$$
\mathcal{M}_{1}^{0}(\Lambda)=\left\{\pi \in \mathcal{M}_{1}(\Lambda): \pi(\mathrm{d} r)=\rho(r) \mathrm{d} r \text { and } 0 \leqslant \rho(r) \leqslant 1 \text { a.e. }\right\},
$$

$\mathcal{M}_{1}^{0}(\Lambda)$ is a closed subset of $\mathcal{M}_{1}(\Lambda)$ endowed with the weak topology and $D\left([0, T], \mathcal{M}_{1}^{0}(\Lambda)\right)$ is a closed subset of $D\left([0, T], \mathcal{M}_{1}(\Lambda)\right)$ for the Skorohod topology. To state next theorem we need the following definition.

Definition. Given a Lebesgue absolutely continuous measure $\rho(r) \mathrm{d} r \in \mathcal{M}_{1}^{0}(\Lambda)$, a sequence of probability measures $\left(\mu_{\gamma}\right)_{\gamma} \geqslant 0$ on $\mathcal{S}_{\gamma}$ is said to correspond to the macroscopic profile $\rho(\cdot)$ if, under $\mu_{\gamma}$ the random variable $\pi^{\gamma}(\cdot)=$ $\gamma^{d} \sum_{x \in \Lambda_{\gamma}} \eta(x) \delta_{\gamma x}(\cdot) \in \mathcal{M}_{1}(\Lambda)$ converges in probability to $\rho(r) \mathrm{d} r$ as $\gamma \downarrow 0$, i.e for any smooth function $G$ and $\delta>0$

$$
\lim _{\gamma \rightarrow 0} \mu_{\gamma}\left\{\left|\gamma^{d} \sum_{x \in \Lambda_{\gamma}} G(\gamma x) \eta(x)-\int_{\Lambda} G(r) \rho(r) \mathrm{d} r\right|>\delta\right\}=0 .
$$

Further we denote by $(J \star v)$ the convolution of $J$ with a function $v$. We recall the theorem proved in [21].

Theorem. [21] Let $d \geqslant 3, \beta>0$ and assume that $D(\rho)$, see (2.19), can be continuously extended to the closed interval $[0,1]$. Let $\mu_{\gamma}$ be a sequence of probability measures on $\mathcal{S}_{\gamma}$ corresponding to the initial profile $\rho_{0}$. Then, $\mathbb{P}$ a.s. the sequence of probability measures $\left(Q_{\mu_{\gamma}}\right)_{\gamma \geqslant 0}$ is tight and all its limit points $Q^{*}$ are concentrated on $\rho(t, r) \mathrm{d} r$, whose densities are weak solutions of the equation

$$
\left\{\begin{array}{l}
\partial_{t} \rho=\sum_{k, m=1}^{d} \partial_{e_{k}}\left\{D_{k, m}(\rho)\left\{\partial_{e_{m}} \rho-\beta \chi(\rho)\left(\partial_{e_{m}} J \star \rho\right)\right\}\right\}, \\
\rho(0, \cdot)=\rho_{0}(\cdot)
\end{array}\right.
$$

satisfying the energy estimate

$$
\int_{0}^{T} \mathrm{~d} s\left(\int_{\Lambda}|\nabla \rho(s, r)|^{2} \mathrm{~d} r\right)<\infty .
$$

Moreover if the $\sigma(\cdot)$, see (2.22), is Lipschitz continuous for $\rho \in[0,1]$, then $\left(Q_{\mu_{\gamma}}\right)_{\gamma \geqslant 0}$ converges weakly, as $\gamma \downarrow 0$, to $Q^{*}$. This limit point is concentrated on the unique weak solution of Eq. (2.26). ${ }^{3}$

Eq. (2.26) can be written as (1.2) where

$$
\mathcal{G}(\rho)=\int\left[g_{0}(\rho(r))-\frac{\beta}{2} \rho(r)(J \star \rho)(r)\right] \mathrm{d} r,
$$

$g_{0}$ is the (strictly convex) free energy density

$$
g_{0}(\rho)=\rho \lambda_{0}(\rho)-p_{0}\left(\lambda_{0}(\rho)\right) ; \quad p_{0}(\lambda)=\mathbb{E}\left[\log \left(1+\mathrm{e}^{\lambda+\alpha(0)}\right)\right] .
$$

\footnotetext{
3 The existence and uniqueness of the weak solution of (2.26) when (2.21) holds, $\sigma(\cdot)$ is Lipschitz continuous for $\rho \in[0,1], \sigma(0)=\sigma(1)=0$ and $J \in C^{2}(\Lambda)$ can be done using standard analysis tools. It has been proven in [9], second reference.
} 
By thermodynamic relations, one has that for $\rho \in[0,1], \lambda_{0}(\rho)$ satisfies

$$
\rho=\frac{\mathrm{d} p_{0}}{\mathrm{~d} \lambda}\left(\lambda_{0}(\rho)\right)=\mathbb{E}\left[\frac{\mathrm{e}^{\lambda_{0}(\rho)+\alpha(0)}}{1+\mathrm{e}^{\lambda_{0}(\rho)+\alpha(0)}}\right] ; \quad \chi(\rho)^{-1}=\lambda_{0}^{\prime}(\rho) .
$$

In order to keep the notation light, we will write the evolution equation in strong form, even when it has to be interpreted in the weak sense. The weak form is obtained by integrating against a function $G \in C^{1,2}([0, T] \times \Lambda)$ and performing the formal integration by parts. We denote by $C^{n, m}([0, T] \times \Lambda)$ the space of functions $G:[0, T] \times \Lambda \rightarrow \mathbb{R}$ with $n$ continuous derivative in time and $m$ continuous derivative in space, being $n$ and $m$ positive integers.

\subsection{The main result}

In the present article we are concerned with the large deviations from the scaling limit described above. To state our result we still need more definitions.

For two functions $f$ and $g$ from $\Lambda$ to $\mathbb{R}$, denote by $\langle f, g\rangle$ the usual inner product in $L^{2}(\Lambda)$. Let $q(\cdot)$ on $\Lambda$ be a function taking values on symmetric positive semidefinite $d \times d$ matrices. For each integrable function $f: \Lambda \rightarrow \mathbb{R}$ with mean zero define

$$
\|f\|_{-1, q(\cdot)}^{2}=\sup _{G}\left\{2\langle G, f\rangle-\int_{\Lambda}(\nabla G(r) \cdot q(r) \nabla G(r)) \mathrm{d} r\right\},
$$

where the supremum is over $C^{\infty}$ functions from $\Lambda$ to $\mathbb{R}$. When $q(r) \equiv \mathbb{1}$ for $r \in \Lambda$, where $\mathbb{1}$ stands for the $d \times d$ identity matrix, we drop the subscript $q$ in (2.30) and denote $\|f\|_{-1, q(\cdot)}^{2} \equiv\|f\|_{-1}^{2}$. For $\mu(\cdot, \cdot) \in D\left([0, T], \mathcal{M}_{1}^{0}(\Lambda)\right)$ with $\mu(t, \mathrm{~d} r)=\rho(t, r) \mathrm{d} r, \int_{\Lambda} \rho(t, r) \mathrm{d} r=\int_{\Lambda} \rho(0, r) \mathrm{d} r$ for all $0 \leqslant t \leqslant T$, define the following functionals

$$
\mathcal{I}_{0}(\mu(\cdot, \cdot)) \equiv \mathcal{I}_{0}(\rho(\cdot, \cdot))=\int_{0}^{T} \int_{\Lambda}\left(\nabla \rho(s, r) \cdot \frac{D(\rho(s, r))}{\chi(\rho(s, r))} \nabla \rho(s, r)\right) \mathrm{d} r \mathrm{~d} s,
$$

and

$$
\begin{aligned}
\mathcal{I}_{\text {dyn }}(\rho(\cdot, \cdot)) & =\frac{1}{2} \int_{0}^{T} \mathrm{~d} t\left\|\partial_{t} \rho-\nabla \cdot(D(\rho)\{\nabla \rho-\beta \chi(\rho) \nabla(J \star \rho)\})\right\|_{-1, \sigma(\rho(t, \cdot))}^{2} \\
& \equiv \frac{1}{2} \int_{0}^{T} \mathrm{~d} t\left\|\frac{\partial \rho}{\partial t}(t, \cdot)-\frac{1}{2} \nabla \cdot\left(\sigma(\rho(t, \cdot)) \nabla \frac{\delta \mathcal{G}}{\delta \rho}(\rho(t, \cdot))\right)\right\|_{-1, \sigma(\rho(t, \cdot))}^{2},
\end{aligned}
$$

where $\sigma(\rho)=2 \chi(\rho) D(\rho)$, see (2.22). A more explicit representation of the functional (2.32) is obtained in Section 3, Lemma 3.1. One consequence of this lemma is that every path $t \rightarrow \mu(t)$ with finite rate function is continuous in the weak topology, $\mu \in C\left([0, T], \mathcal{M}_{1}^{0}(\Lambda)\right)$. We are now ready to define the large deviations rate function. For each $\mu(\cdot, \cdot) \in D\left([0, T], \mathcal{M}_{1}(\Lambda)\right)$ let

$$
\mathcal{I}(\mu(\cdot, \cdot))= \begin{cases}\mathcal{I}_{\text {init }}(\rho(0, \cdot))+\mathcal{I}_{\text {dyn }}(\rho(\cdot, \cdot)) & \text { if }(\mu(\cdot, \cdot))<\infty, \\ \infty & \text { otherwise, }\end{cases}
$$

where $\mathcal{I}_{\text {init }}(\rho(0, \cdot))$ is the large deviation rate for the initial profile $\rho(0, \cdot)$ which depends on the choice of the initial measure. If we were to start with a Bernoulli product measure $\nu_{\gamma}^{\rho_{0}(\cdot)}$ with $\rho_{0}(\gamma x)$ as the probability of a site $x \in \Lambda_{\gamma}$ being occupied then

$$
\mathcal{I}_{\text {init }}(\rho(0, \cdot))=\int_{\Lambda} \mathrm{d} r h\left(\rho(0, r), \rho_{0}(r)\right),
$$

where

$$
h(a, b)=a \log \frac{a}{b}+(1-a) \log \frac{1-a}{1-b} .
$$


For other suitable initial conditions one considers the corresponding rate function for the initial profile. In the following we assume for simplicity to start with a Bernoulli product measure, then $\mathcal{I}_{\text {init }}$ is the one given in (2.34). The main result of this paper is that $\left(Q_{\nu_{\gamma}}^{\rho_{0}}\right)_{\gamma>0}$ satisfies the large deviation principle with rate function $\mathcal{I}$.

Theorem 2.1. Let $d \geqslant 3, \beta \geqslant 0$. Assume that $D(\cdot) \in C^{1, a}([0,1])^{4}$ and the initial measure is the Bernoulli product measure $v_{\gamma}^{\rho_{0}(\cdot)}$. For each closed set $\mathcal{C} \subset D\left([0, T], \mathcal{M}_{1}(\Lambda)\right), \mathbb{P}$ a.s.

$$
\limsup _{\gamma \rightarrow 0} \gamma^{d} \log \left(Q_{\nu_{\gamma}}^{\rho_{0}}(\mathcal{C})\right) \leqslant-\inf _{\mu(\cdot, \cdot) \in \mathcal{C}} \mathcal{I}(\mu(\cdot, \cdot))
$$

and for each open set $\mathcal{O} \subset D\left([0, T], \mathcal{M}_{1}(\Lambda)\right), \mathbb{P}$ a.s.

$$
\liminf _{\gamma \rightarrow 0} \gamma^{d} \log \left(Q_{\nu_{\gamma}^{\rho_{0}}}(\mathcal{O})\right) \geqslant-\inf _{\mu(\cdot, \cdot) \in \mathcal{O}} \mathcal{I}(\mu(\cdot, \cdot)),
$$

where $\mathcal{I}$ is defined in (2.33). The rate function $\mathcal{I}(\mu(\cdot, \cdot))$ is lower semicontinuous and has compact level sets.

The requirement $D(\cdot) \in C^{1, a}([0,1])$ is needed to complete the proof of lower bound, i.e its extension. If $D(\cdot)$ is taken Lipschitz continuous for $\rho \in(0,1)$ then $(2.36)$ holds for paths in $\mathcal{D}^{0}$, see Section 6 . We will show $(2.35)$ in Section 5, (2.36) in Section 6 and 7, the properties of the rate function in Section 3. When $\beta=0$, Theorem 2.1 states the large deviation principle for the empirical measures of the unperturbed process, i.e the one considered in [8]. The corresponding rate function is the one obtained setting $\beta=0$ in (2.32).

Notation warning. Through the text $J, \beta, \Phi$, and $A$ (the bound on $\alpha(x)$ ) will be kept fixed. We therefore avoid to write explicitly the dependence on these quantities. Further, to have lighter notation we omit to write the explicit dependence on the random field $\alpha$. This should not cause any confusion but the reader should keep it in mind. The notation $\mathrm{O}_{u}(n)$ should be understood in the standard sense of $\mathrm{O}(n)$, but uniformly with respect to the disorder $\alpha$, configurations $\eta$, and history of the process. Finally we denote by $C$ a constant that might depend on $J, \beta$, dimension and $A$ which may change from one occurrence to the next.

\section{Properties of the rate function}

In this section we prove a representation result for $\mathcal{I}_{\text {dyn }}$, see Lemma 3.1, its lower semicontinuity, see Theorem 3.4 and the compactness of its level sets. This last property is a consequence of the estimates proven in Theorem 3.3. Note that if $\mathcal{I}_{0}(\rho(\cdot, \cdot))<\infty$ the functional $\mathcal{I}_{\text {dyn }}$ has, by duality, a variational formula. Let $\rho(\cdot, \cdot)$ so that $\mathcal{I}_{0}(\rho(\cdot, \cdot))<\infty$, define for $G \in C^{1,2}([0, T] \times \Lambda)$, the linear functional

$$
\begin{aligned}
\ell_{G}(\rho(\cdot, \cdot))= & \int_{\Lambda} G(T, r) \rho(T, r) \mathrm{d} r-\int_{\Lambda} G(0, r) \rho(0, r) \mathrm{d} r-\iint_{0}\left(\partial_{t} G\right)(t, r) \rho(t, r) \mathrm{d} r \mathrm{~d} t \\
& +\int_{0}^{T} \int_{\Lambda}(D(\rho)\{\nabla \rho-\beta \chi(\rho) \nabla(J \star \rho)\} \cdot \nabla G) \mathrm{d} r \mathrm{~d} t,
\end{aligned}
$$

and

$$
\mathcal{J}_{G}(\rho)=\ell_{G}(\rho)-\frac{1}{2} \int_{0}^{T} \int_{\Lambda}(\sigma(\rho) \nabla G \cdot \nabla G) \mathrm{d} r \mathrm{~d} t .
$$

Then, see (2.30)

$$
\mathcal{I}_{\text {dyn }}(\rho(\cdot, \cdot))=\sup _{G}\left\{\mathcal{J}_{G}(\rho(\cdot, \cdot))\right\}
$$

\footnotetext{
4 The Holder space $C^{k, a}([0,1])$ is defined as the subspace of $C^{k}([0,1])$ consisting of functions whose $k$-th order derivative is Holder continuous with exponent $0<a<1$ in $(0,1)$.
} 
where the sup is over $G \in C^{1,2}([0, T] \times \Lambda)$. From (3.2) arguing as in [15], one obtains an explicit representation formula for $\mathcal{I}_{\text {dyn }}(\rho(\cdot, \cdot))$. Let $\mu(\cdot, \cdot) \in D\left([0, T], \mathcal{M}_{1}^{0}(\Lambda)\right)$ with $\mu(t, \mathrm{~d} r)=\rho(t, r) \mathrm{d} r$. Define in $C^{1,2}([0, T] \times \Lambda)$ the inner product

$$
\langle G, \Phi\rangle_{\rho}=\frac{1}{2} \int_{0}^{T} \mathrm{~d} t \int_{\Lambda} \mathrm{d} r(\nabla G(t, r) \cdot \sigma(\rho(t, r)) \nabla \Phi(t, r)) .
$$

Denote by $\mathcal{N}(\rho)$ the kernel of this inner product and by $\mathcal{H}_{1}(\rho)$ the Hilbert space obtained by completing $C^{1,2}([0, T] \times$ $\Lambda)\left.\right|_{\mathcal{N}(\rho)}$.

Lemma 3.1. Assume that $\mathcal{I}_{\mathrm{dyn}}(\rho(\cdot, \cdot))<\infty$. There exists $S \in \mathcal{H}_{1}(\rho)$ so that

$$
\mathcal{I}_{\text {dyn }}(\rho(\cdot, \cdot))=\frac{1}{8} \int_{0}^{T} \mathrm{~d} t \int_{\Lambda} \mathrm{d} r(\nabla S(t, r) \cdot \sigma(\rho(t, r)) \nabla S(t, r))
$$

and $\rho$ satisfies in the weak sense the equation

$$
\partial_{t} \rho=\sum_{k, m=1}^{d} \partial_{e_{k}}\left\{D_{k, m}(\rho)\left\{\partial_{e_{m}} \rho-\beta \chi(\rho)\left(\partial_{e_{m}} J \star \rho\right)-\chi(\rho)\left(\partial_{e_{m}} S\right)\right\}\right\} .
$$

The proof is similar to the one done in [15], see also [14]. One shows first that $\ell .(\rho(\cdot, \cdot))$ is a linear functional bounded in $\mathcal{H}_{1}(\rho)$, then extends $\ell .(\rho(\cdot, \cdot))$ to $\mathcal{H}_{1}(\rho)$ and, by Riesz' representation theorem, there exists $S \in \mathcal{H}_{1}(\rho)$ so that for each $G \in \mathcal{H}_{1}(\rho)$

$$
\ell_{G}(\rho(\cdot, \cdot))=\frac{1}{2} \int_{0}^{T} \mathrm{~d} t \int_{\Lambda} \mathrm{d} r(\nabla G(t, r) \cdot \sigma(\rho(t, r)) \nabla S(t, r)) .
$$

Finally from (3.2) one deduces (3.4) and (3.5).

There are two parts in the definition of the rate function $\mathcal{I}$, the static part, $\mathcal{I}_{\text {init }}$, corresponding to large deviations from the initial measure and the dynamic part, $\mathcal{I}_{\text {dyn }}$, due to the stochastic character of the evolution. The static part is clearly convex and lower semicontinuous. To prove the lower semicontinuity of the dynamic part we need the following result, stated in Lemma 4.2 of [26], which we recall:

Lemma 3.2. Let $\left(\rho_{n}\right)$ be a sequence of functions in $L^{\infty}([0, T] \times \Lambda)$ such that uniformly on $n$,

$$
\int_{0}^{T} \int_{\Lambda}\left|\nabla \rho_{n}\right|^{2} \mathrm{~d} r \mathrm{~d} t+\int_{0}^{T}\left\|\frac{\partial \rho_{n}}{\partial t}\right\|_{-1}^{2} \mathrm{~d} t<C
$$

where $\|\cdot\|_{-1}$ has been defined after (2.30) and $C$ is a positive constant. Suppose that $\rho \in L^{\infty}([0, T] \times \Lambda)$ and that $\rho_{n} \rightarrow \rho$ weakly in $L^{2}([0, T] \times \Lambda)$. Then $\rho_{n} \rightarrow \rho$ strongly in $L^{1}([0, T] \times \Lambda)$.

The proof of this lemma use some relative compactness arguments in $L^{2}([0, T] \times \Lambda)$. We refer for its proof to [26], Lemma 4.2. Further we show the following estimates.

Theorem 3.3. There is a positive constant $C_{1}$ so that $\mathcal{I}_{\mathrm{dyn}}(\rho(\cdot, \cdot))$ satisfies the bounds

$$
\begin{aligned}
& \mathcal{I}_{0}(\rho) \leqslant C_{1}+8 \mathcal{I}_{\text {dyn }}(\rho)+2 \beta^{2} \iint_{0}^{T}(\sigma(\rho) \nabla(J \star \rho) \cdot \nabla(J \star \rho)) \mathrm{d} r \mathrm{~d} t, \\
& \int_{0}^{T}\left\|\partial_{t} \rho\right\|_{-1, \sigma(\rho(t, \cdot))}^{2} \mathrm{~d} t \leqslant 2 C_{1}+20 \mathcal{I}_{\text {dyn }}(\rho)+5 \beta^{2} \int_{0}^{T} \int_{\Lambda}(\sigma(\rho) \nabla(J \star \rho) \cdot \nabla(J \star \rho)) \mathrm{d} r \mathrm{~d} t .
\end{aligned}
$$


Proof. When $\mathcal{I}_{\text {dyn }}(\rho)=\infty$, the two inequalities are trivially verified. Suppose then that $\mathcal{I}_{\text {dyn }}(\rho)<\infty$. This implies that $\mathcal{I}_{0}(\rho)<\infty$, see (2.31), and that $\int_{0}^{T}\left\|\partial_{t} \rho\right\|_{-1, \sigma(\rho(t, \cdot))}^{2} \mathrm{~d} t<\infty$. Recall, see (2.28), that $g_{0}(\rho)$ is the bounded, strictly convex free energy density of the system with only short range interaction, $g_{0}^{\prime}(\rho)=\lambda_{0}(\rho), \lambda_{0}^{\prime}(\rho)=1 / \chi(\rho)$ and $\sigma(\rho) \nabla\left(g_{0}^{\prime}(\rho) / 2\right)=D(\rho) \nabla \rho$. Since $\mathcal{I}_{\text {dyn }}(\rho)$ is bounded, adding and subtracting the same quantity, taking in account (3.1), we have that

$$
\begin{aligned}
\frac{1}{2} \int_{0}^{T} \mathrm{~d} t\left(\frac{\partial}{\partial t} \int_{\Lambda} g_{0}(\rho(t, r)) \mathrm{d} r\right)= & \left.\ell_{g_{0}^{\prime}(\rho) / 2}(\rho)-\frac{1}{2} \int_{0}^{T} \int_{\Lambda}\left(\nabla \frac{g_{0}^{\prime}(\rho)}{2} \cdot \sigma(\rho) \nabla \frac{g_{0}^{\prime}(\rho)}{2}\right) \mathrm{d} r \mathrm{~d} t\right\} \\
& -\iint_{0}^{T}\left(D(\rho)\{\nabla \rho-\beta \chi(\rho) \nabla(J \star \rho)\} \cdot \nabla \frac{g_{0}^{\prime}}{2}(\rho)\right) \mathrm{d} r \mathrm{~d} t \\
& +\frac{1}{2} \int_{0}^{T} \int_{\Lambda}\left(\nabla \frac{g_{0}^{\prime}(\rho)}{2} \cdot \sigma(\rho) \nabla \frac{g_{0}^{\prime}(\rho)}{2}\right) \mathrm{d} r \mathrm{~d} t .
\end{aligned}
$$

Hence, since (3.2) and, by assumption, $\mathcal{I}_{0}(\rho)$ is finite, one concludes that

$$
\frac{1}{2} \int_{0}^{T} \mathrm{~d} t\left(\frac{\partial}{\partial t} \int_{\Lambda} g_{0}(\rho(t, r)) \mathrm{d} r\right) \leqslant \mathcal{I}_{\text {dyn }}(\rho)-\frac{1}{4} \mathcal{I}_{0}(\rho)+\frac{\beta}{2} \int_{0}^{T} \int_{\Lambda}(D(\rho) \nabla \rho \cdot \nabla(J \star \rho)) \mathrm{d} r \mathrm{~d} t .
$$

Since $D(\cdot)$ is a symmetric matrix, positive defined, and we have $x y \leqslant \frac{1}{2}\left[a x^{2}+\frac{1}{a} y^{2}\right]$ for any $a>0$ we obtain

$$
\beta \iint_{0}^{T}(D(\rho) \nabla \rho \cdot \nabla(J \star \rho)) \mathrm{d} r \mathrm{~d} t \leqslant \frac{\beta}{2 a} \mathcal{I}_{0}(\rho)+\frac{\beta a}{4} \int_{0}^{T} \int_{\Lambda}(\sigma(\rho) \nabla(J \star \rho) \cdot \nabla(J \star \rho)) \mathrm{d} r \mathrm{~d} t .
$$

Choosing $a=2 \beta$ and inserting this last inequality in (3.10), we obtain

$$
\frac{1}{2} \int_{0}^{T} \mathrm{~d} t\left(\frac{\partial}{\partial t} \int_{\Lambda} g_{0}(\rho(t, r)) \mathrm{d} r\right) \leqslant \mathcal{I}_{\text {dyn }}(\rho)-\frac{1}{8} \mathcal{I}_{0}(\rho)+\frac{\beta^{2}}{4} \iint_{0}^{T} \int_{\Lambda}(\sigma(\rho) \nabla(J \star \rho) \cdot \nabla(J \star \rho)) \mathrm{d} r \mathrm{~d} t .
$$

On the other hand, we have

$$
\left|\int_{0}^{T} \mathrm{~d} t\left(\frac{\partial}{\partial t} \int_{\Lambda} g_{0}(\rho(t, r)) \mathrm{d} r\right)\right|=\left|\int_{\Lambda} g_{0}(\rho(T, r)) \mathrm{d} r-\int_{\Lambda} g_{0}(\rho(0, r)) \mathrm{d} r\right|=C_{1}^{\prime}
$$

for some positive constant $C_{1}^{\prime}$. Denoting $C_{1}=8 C_{1}^{\prime}$ we easily obtain from (3.11) the inequality (3.7). The (3.8) is obtained from (3.7). Namely, from the definition of $\mathcal{I}_{\text {dyn }}$, see (2.32), we have

$$
\begin{aligned}
\int_{0}^{T} \mathrm{~d} t\left\|\frac{\partial \rho}{\partial t}\right\|_{-1, \sigma(\rho(t, \cdot))}^{2} \leqslant & 2 \int_{0}^{T} \mathrm{~d} t\left\|\frac{\partial \rho}{\partial t}-\frac{1}{2} \nabla \cdot\left(\sigma(\rho(t, \cdot)) \nabla \frac{\delta \mathcal{G}}{\delta \rho}(\rho(t, \cdot))\right)\right\|_{-1, \sigma(\rho(t, \cdot))}^{2} \\
& +\frac{1}{2} \int_{0}^{T} \mathrm{~d} t\left\|\nabla \cdot\left(\sigma(\rho(t, \cdot)) \nabla \frac{\delta \mathcal{G}}{\delta \rho}(\rho(t, \cdot))\right)\right\|_{-1, \sigma(\rho(t, \cdot))}^{2}
\end{aligned}
$$

Taking in account that

$$
2 \int_{0}^{T} \mathrm{~d} t\|\nabla \cdot(D(\rho(t, \cdot)) \nabla \rho(t, \cdot))\|_{-1, \sigma(\rho(t, \cdot))}^{2}=\mathcal{I}_{0}(\rho),
$$


we have

$$
\begin{aligned}
& \frac{1}{2} \int_{0}^{T} \mathrm{~d} t\left\|\nabla \cdot\left(\sigma(\rho(t, \cdot)) \nabla \frac{\delta \mathcal{G}}{\delta \rho}(\rho(t, \cdot))\right)\right\|_{-1, \sigma(\rho(t, \cdot))}^{2} \\
& \quad \leqslant 4 \int_{0}^{T} \mathrm{~d} t\|\nabla \cdot(D(\rho(t, \cdot)) \nabla \rho(t, \cdot))\|_{-1, \sigma(\rho(t, \cdot))}^{2}+\beta^{2} \int_{0}^{T} \mathrm{~d} t\|\nabla \cdot(\sigma(\rho(t, \cdot)) \nabla(J \star \rho)(t, \cdot))\|_{-1, \sigma(\rho(t, \cdot))}^{2} \\
& \quad=2 \mathcal{I}_{0}(\rho)+\beta^{2} \int_{0}^{T} \mathrm{~d} t\|\nabla \cdot(\sigma(\rho(t, \cdot)) \nabla(J \star \rho)(t, \cdot))\|_{-1, \sigma(\rho(t, \cdot))}^{2} \cdot
\end{aligned}
$$

Then the inequality (3.12) becomes

$$
\int_{0}^{T} \mathrm{~d} t\left\|\frac{\partial \rho}{\partial t}\right\|_{-1, \sigma(\rho(t, \cdot))}^{2} \leqslant 4 \mathcal{I}_{\text {dyn }}(\rho)+2 \mathcal{I}_{0}(\rho)+\beta^{2} \int_{0}^{T} \mathrm{~d} t\|\nabla \cdot(\sigma(\rho(t, \cdot)) \nabla(J \star \rho)(t, \cdot))\|_{-1, \sigma(\rho(t, \cdot)}^{2} .
$$

Applying to $\mathcal{I}_{0}(\rho)$ the estimate (3.7) we obtain (3.8).

Theorem 3.4. The functional $\mathcal{I}_{\mathrm{dyn}}(\rho(\cdot, \cdot))$ is lower semicontinuous in $D\left([0, T], \mathcal{M}_{1}(\Lambda)\right)$.

Proof. Let $\pi_{n} \in D\left([0, T], \mathcal{M}_{1}(\Lambda)\right)$ be a sequence converging to $\pi$ in $D\left([0, T], \mathcal{M}_{1}(\Lambda)\right)$ :

$$
\lim _{n \rightarrow \infty} \int_{\Lambda} \mathrm{d} r f(r) \pi_{n}(t, \mathrm{~d} r)=\int_{\Lambda} \mathrm{d} r f(r) \pi(t, \mathrm{~d} r) \quad \text { for any } f \in C^{0}(\Lambda)
$$

for almost all $t \in[0, T]$. We need to show that

$$
\liminf _{n \rightarrow \infty} \mathcal{I}_{\text {dyn }}\left(\pi_{n}(\cdot, \cdot)\right) \geqslant \mathcal{I}_{\text {dyn }}(\pi(\cdot, \cdot)) .
$$

We can always suppose that $\mathcal{I}_{\text {dyn }}\left(\pi_{n}(\cdot, \cdot)\right)$ is bounded uniformly on $n$. This implies $\pi_{n}(\cdot, \cdot) \in D\left([0, T], \mathcal{M}_{1}^{0}(\Lambda)\right)$, and $\pi_{n}(t, \mathrm{~d} r)=\rho_{n}(t, r) \mathrm{d} r$ for almost all $t \in[0, T]$. Since $D\left([0, T], \mathcal{M}_{1}^{0}(\Lambda)\right)$ is a closed subset of $D\left([0, T], \mathcal{M}_{1}(\Lambda)\right)$ then $\pi(t, \mathrm{~d} r)=\rho(t, r) \mathrm{d} r$ for almost all $t \in[0, T]$. Further, since (3.7), there exists a constant $C>0$ so that

$$
\mathcal{I}_{0}\left(\rho_{n}(\cdot, \cdot)\right) \leqslant C \quad \text { for any } n ; \quad \mathcal{I}_{0}(\rho(\cdot, \cdot)) \leqslant C
$$

and from (3.8)

$$
\int_{0}^{T} \mathrm{~d} t\left\|\frac{\partial \rho_{n}}{\partial t}\right\|_{-1, \sigma\left(\rho_{n}(t, \cdot)\right)}^{2} \leqslant C \quad \text { for any } n .
$$

Since (3.15) implies that $\rho_{n} \rightarrow \rho$ weakly in $L^{2}([0, T] \times \Lambda),(3.17)$ and (3.18) hold, then from Lemma 3.2 we deduce that $\rho_{n}$ converges strongly to $\rho$ in $L^{1}([0, T] \times \Lambda)$. From (3.2), to prove the lower semicontinuity of $\mathcal{I}_{\text {dyn }}(\rho)$, it is enough to show that for any function $G \in C^{1,2}([0, T] \times \Lambda)$, we have

$$
\mathcal{J}_{G}(\rho) \leqslant \liminf _{n \rightarrow \infty} \mathcal{J}_{G}\left(\rho_{n}\right) .
$$

Note that

$$
\int_{0}^{T} \int_{\Lambda}(D(\rho) \nabla \rho \cdot \nabla G) \mathrm{d} r \mathrm{~d} t=\sum_{1 \leqslant i, j \leqslant d} \iint_{0}^{T} \int_{\Lambda} \hat{d}_{i, j}(\rho(t, r)) \partial_{e_{i}, e_{j}}^{2} G(t, r) \mathrm{d} r \mathrm{~d} t,
$$

where $\hat{d}_{i, j}$ stands for the integral of $D_{i, j}$ :

$$
\hat{d}_{i, j}(m)=\int_{0}^{m} D_{i, j}\left(m^{\prime}\right) \mathrm{d} m^{\prime}, \quad \text { for } m \in[0,1]
$$


Taking in account the different terms of $\mathcal{J}_{G},(3.20)$ and using the continuity of functions $m \rightarrow \hat{d}_{i, j}(m), m \rightarrow \sigma_{i, j}(m)$ and $m \rightarrow \chi(m)$, it is easy to see that in order to prove (3.19) we need only the strong convergence of $\rho_{n}$ to $\rho$ in $L^{1}([0, T] \times \Lambda)$, which we do have thanks to Lemma 3.2 .

\section{Macroscopic limit for system with weak random driving forces}

For $\ell \in \mathbb{N}$, denote by $\eta^{\ell}(x)$ the average density of $\eta$ in a cube of width $2 \ell+1$ centered in $x$

$$
\eta^{\ell}(x)=\frac{1}{(2 \ell+1)^{d}} \sum_{y:|y-x| \leqslant \ell} \eta(y) .
$$

For a function $G$ on $\Lambda$ and $e \in \mathcal{E}$ denote by $\partial_{e}^{\gamma} G$ the discrete derivative in the direction $e$ and by $\nabla^{\gamma} G$ the discrete gradient

$$
\begin{aligned}
& \left(\partial_{e}^{\gamma} G\right)(\gamma x)=\gamma^{-1}[G(\gamma(x+e))-G(\gamma x)], \\
& \left(\nabla^{\gamma} G\right)(\gamma x)=\left(\left(\partial_{e_{1}}^{\gamma} G\right)(\gamma x), \ldots,\left(\partial_{e_{d}}^{\gamma} G\right)(\gamma x)\right) .
\end{aligned}
$$

Further, let $V(\cdot, \cdot) \in C^{1,2}([0, T] \times \Lambda)$ and $v(\eta, \alpha) \equiv v\left(\eta^{\ell}(0), \eta, \alpha\right)=\left(v_{1}\left(\eta^{\ell}(0), \eta, \alpha\right), \ldots, v_{d}\left(\eta^{\ell}(0), \eta, \alpha\right)\right) \in \mathbb{G}^{d}$. We assume that the $v_{k}, k=1, \ldots, d$, have support in a cube of side $\ell$ centered at the origin and that they are smooth functions with respect to the first variable $\eta^{\ell}(0)$. To short notation we do not write explicitly the dependence of $v_{k}$ on the local empirical density $\eta^{\ell}(0)$. Let

$$
H_{\gamma}^{V, v}(\eta)=-\sum_{x \in \Lambda_{\gamma}}\left[V(t, \gamma x) \eta(x)+\gamma\left(\nabla^{\gamma} V(t, \gamma x) \cdot \tau_{x} v(\eta, \alpha)\right)\right]
$$

Define at time $t, 0 \leqslant t \leqslant T$, the following generator of a time inhomogeneous Markov process on $\mathcal{S}_{\gamma}$

$$
\left(\mathcal{L}_{\gamma}^{V, v} f\right)(\eta)=\sum_{e \in \mathcal{E}} \sum_{x \in \Lambda_{\gamma}} C_{\gamma}^{V, v}(x, x+e ; \eta)\left[\left(\nabla_{x, x+e} f\right)(\eta)\right]
$$

where the rate function $C_{\gamma}^{V, v}$ is defined through the rate $C_{\gamma}$ and $H_{\gamma}^{V, v}$ by

$$
C_{\gamma}^{V, v}(x, x+e ; \eta)=C_{\gamma}(x, x+e ; \eta) \exp \left(-\frac{1}{2}\left(\nabla_{x, x+e} H_{\gamma}^{V, v}\right)(\eta)\right)
$$

Let $\mu_{\gamma}$ be a sequence of probability measures on $\mathcal{S}_{\gamma}$ corresponding to a macroscopic profile $\rho_{0}$, see (2.25). Let $\mathbf{P}_{\mu_{\gamma}, v}^{V, v}$ be the law of the inhomogeneous Markov process $\left(\eta_{t}\right)_{t \in[0, T]}$ on the path space $D\left([0, T], \mathcal{S}_{\gamma}\right)$ with generator $\gamma^{-2} \mathcal{L}_{\gamma}^{V, v}$ and initial distribution $\mu_{\gamma}$. Let $\left(Q_{\mu_{\gamma}}^{V, v}\right)$ be the measure of the process $\left(\pi_{t}^{\gamma}\right)_{t \in[0, T]}$ on the state space $D\left([0, T], \mathcal{M}_{1}(\Lambda)\right)$ induced from $\mathbf{P}_{\mu_{\gamma}}^{V, v}$.

Theorem 4.1. Let $d \geqslant 3$. Assume that $D(\rho)$, defined in (2.19) can be continuously extended to the closed interval $[0,1]$ and that $\sigma(\rho)$ is Lipschitz continuous for $\rho \in[0,1]$. Let $V$ and $v$ be as described above, then $\mathbb{P}$ a.s., the sequence of probability measures $\left(Q_{\mu_{\gamma}}^{V, v}\right)_{\gamma} \geqslant 0$ converges as $\gamma \downarrow 0$, to $Q^{V}$. This limit point is concentrated on the unique weak solution of the following equation

$$
\begin{aligned}
& \left\{\begin{array}{l}
\partial_{t} \rho=\sum_{k, m=1}^{d} \partial_{e_{k}}\left\{D_{k, m}(\rho)\left\{\partial_{e_{m}} \rho-\beta \chi(\rho)\left(\partial_{e_{m}} J \star \rho\right)-\chi(\rho)\left(\partial_{e_{m}} V\right)\right\}\right\}, \\
\rho(0, \cdot)=\rho_{0}(\cdot) .
\end{array}\right. \\
& \int_{0}^{T} \mathrm{~d} s\left(\int_{\Lambda}|\nabla \rho(s, u)|^{2} \mathrm{~d} u\right)<\infty .
\end{aligned}
$$

Remark. One can write Eq. (4.6) as

$$
\frac{\partial \rho}{\partial t}=\frac{1}{2} \nabla \cdot\left(\sigma(\rho) \nabla\left\{\frac{\delta \mathcal{G}}{\delta \rho}-V(t, \cdot)\right\}\right)
$$


where $\mathcal{G}$ is the functional defined in (2.27). Note that the macroscopic limit does not depend on the choice of the local functions $v$.

Theorem 4.1 is slight more general of the result stated in [21] due to the term with the local functions $v$ in (4.3). Namely the system with only the weak driving force coming from $\sum_{x \in \Lambda_{\gamma}} V(t, \gamma x) \eta(x)$ could be treated as the term coming from Kac's interaction in [21]. Here we must show that the perturbation coming from adding local random functions is not felt at macroscopic level. Looking at the second term in (4.3) since the presence of $\gamma$ one could think that this part of the perturbation is of higher order and therefore it would simple disappear in the macroscopic limit. But from (4.13) one sees that the weak driving force coming from the second term in (4.3) is of the same order of the weak driving force coming from the first term in (4.3). To take this in account and since the methods to obtain the law of large numbers are close to the methods to obtain estimates for upper and lower bounds of large deviations we outline the proof of Theorem 4.1. Tightness of $\left(Q_{\mu_{\gamma}}^{V, v}\right)_{\gamma} \geqslant 0$ and energy estimates can be obtained in the same way as in [21], see Proposition 3.1 and Proposition 3.3 there. We will prove only the identification of the support of the $Q^{V, v}$ as weak solution of (4.6).

\subsection{Some basic lemmas}

In this section we prove some results needed either to identify the limit equation (4.6), either to prove large deviation principle. It is well known that one of the main steps in the derivation of a large deviation principle for the empirical density is a superexponential estimate which allows the replacement of local functions by functionals of the empirical density in the large deviations regime. We recall in Lemma 4.3 the superexponential estimate for the process generated by $\gamma^{-2} \mathcal{L}_{\gamma}$ proven in [21] and, as consequence of this, we show in Lemma 4.4 that the superexponential estimate holds also for the process generated by $\gamma^{-2} \mathcal{L}_{\gamma}^{V, v}$. The following lemma shows that the exchange rates of $\mathcal{L}_{\gamma}^{V, v}$ are a perturbation of the ones of $\mathcal{L}_{\gamma}$. From Lemma 3.4 in [21] they are also a perturbation of the exchange rates of the $\mathcal{L}_{\gamma}^{0}$ process. Denote

$$
\Gamma_{v_{k}}(\eta)=\sum_{z} \tau_{z} v_{k}(\eta, \alpha)
$$

and the current of the DLG process, i.e the one generated by $\mathcal{L}_{\gamma}^{0}$, by

$$
\mathbf{J}_{x, x+e}^{0} \equiv \mathbf{J}_{x, x+e}^{0}(\eta, \alpha)=C^{0}(x, x+e ; \eta)[\eta(x)-\eta(x+e)] .
$$

Lemma 4.2. For every $x \in \Lambda_{\gamma}, e \in \mathcal{E}, \eta \in \mathcal{S}_{\gamma}, 0 \leqslant t \leqslant T$, for $\gamma \in(0,1)$, for all $\alpha \in \Omega_{D}$

$$
\begin{aligned}
C_{\gamma}^{V, v}(x, x+e ; \eta)= & C_{\gamma}(x, x+e ; \eta)+\frac{\gamma}{2} \mathbf{J}_{x, x+e}^{0}\left(\left(\nabla^{\gamma} V\right)(t, \gamma x) \cdot e\right) \\
& +\frac{\gamma}{2} C^{0}(x, x+e ; \eta)\left\{\sum_{k=1}^{d}\left(\partial_{e_{k}}^{\gamma} V\right)(t, \gamma x)\left(\nabla_{x, x+e} \Gamma_{v_{k}}\right)(\eta)\right\}+\mathrm{O}_{u}\left(\gamma^{2}\right) \mathrm{O}_{u}\left(\ell^{d}\right) . \\
C_{\gamma}^{V, v}(x, x+e ; \eta)= & C^{0}(x, x+e ; \eta)+\gamma \Phi^{\prime}\left(\left(\nabla_{x, x+e} H_{0}^{\alpha}\right)(\eta)\right)[\eta(x+e)-\eta(x)]\left[\beta\left(\partial_{e}^{\gamma} J \star \pi_{\gamma}\right)(\gamma x)\right] \\
& +\frac{\gamma}{2} \mathbf{J}_{x, x+e}^{0}\left(\left(\nabla^{\gamma} V\right)(t, \gamma x) \cdot e\right) \\
& +\frac{\gamma}{2} C^{0}(x, x+e ; \eta)\left\{\sum_{k=1}^{d}\left(\partial_{e_{k}}^{\gamma} V\right)(t, \gamma x)\left(\nabla_{x, x+e} \Gamma_{v_{k}}\right)(\eta)\right\}+\mathrm{O}_{u}\left(\gamma^{2}\right) \mathrm{O}_{u}\left(\ell^{d}\right),
\end{aligned}
$$

provided $J$ as in (2.3), $\Phi$ defined in (2.10), $V$, $v$ as in (4.3).

Proof. We have

$$
C_{\gamma}^{V, v}(x, x+e ; \eta)-C_{\gamma}(x, x+e ; \eta)=C_{\gamma}(x, x+e ; \eta)\left\{\exp \left(-\frac{1}{2}\left(\nabla_{x, x+e} H_{\gamma}^{V, v}\right)(\eta)\right)-1\right\}
$$


and

$$
\begin{aligned}
\nabla_{x, x+e} H_{\gamma}^{V, v} & =\gamma\left(\partial_{e}^{\gamma} V\right)(t, \gamma x)[\eta(x+e)-\eta(x)]-\gamma \sum_{k} \sum_{|z-x| \leqslant \ell}\left(\partial_{e_{k}}^{\gamma} V\right)(t, \gamma z)\left(\nabla_{x, x+e} \tau_{z} v_{k}\right)(\eta, \alpha) \\
& =\gamma\left(\partial_{e}^{\gamma} V\right)(t, \gamma x)[\eta(x+e)-\eta(x)]-\gamma \sum_{k}\left(\partial_{e_{k}}^{\gamma} V\right)(t, \gamma x)\left(\nabla_{x, x+e} \Gamma_{v_{k}}\right)(\eta)+\mathrm{O}_{u}\left(\gamma^{2}\right) \mathrm{O}_{u}\left(\ell^{d}\right) .
\end{aligned}
$$

Taylor expanding the function $\mathrm{e}^{u}-1$ gives

$$
\begin{gathered}
C_{\gamma}^{V, v}(x, x+e ; \eta)-C_{\gamma}(x, x+e ; \eta)=C^{0}(x, x+e ; \eta)\left\{-\frac{1}{2}\left(\nabla_{x, x+e} H_{\gamma}^{V, v}\right)(\eta)\right\} \\
+\left\{C_{\gamma}(x, x+e ; \eta)-C^{0}(x, x+e ; \eta)\right\}\left\{-\frac{1}{2}\left(\nabla_{x, x+e} H_{\gamma}^{V, v}\right)(\eta)\right\}+\mathrm{O}_{u}\left(\gamma^{2}\right)
\end{gathered}
$$

and then (4.11) follows. Taylor expanding $\Phi$ since $\nabla_{x, x+e} H_{\gamma}$ is a perturbation of $\nabla_{x, x+e} H_{0}^{\alpha}$ gives

$$
C_{\gamma}(x, x+e ; \eta)-C^{0}(x, x+e ; \eta)=\gamma \Phi^{\prime}\left(\left(\nabla_{x, x+e} H_{0}^{\alpha}\right)(\eta)\right)[\eta(x+e)-\eta(x)]\left[\beta\left(\partial_{e}^{\gamma} J \star \pi_{\gamma}\right)(\gamma x)\right]+\mathrm{O}_{u}\left(\gamma^{2}\right) .
$$

Inserting (4.15) and (4.13) in (4.14) gives (4.12).

For any cylinder bounded function $g: \mathcal{S} \times \Omega_{D} \rightarrow \mathbb{R}$ define $\tilde{g}:[0,1] \rightarrow \mathbb{R}$ by

$$
\tilde{g}(\rho) \equiv \mathbb{E}\left[\mathbf{E}^{\mu^{\alpha, \lambda_{0}(\rho)}}[g]\right]
$$

and for any fixed $b>0$ set

$$
\mathcal{B}_{b \gamma^{-1}}(\eta, \alpha)=\left|\frac{1}{\left(2 b \gamma^{-1}+1\right)^{d}} \sum_{|y| \leqslant b \gamma^{-1}}\left[\tau_{y} g(\alpha, \eta)-\tilde{g}\left(\eta^{\left[b \gamma^{-1}\right]}(0)\right)\right]\right| .
$$

We recall the superexponential estimate for the $\mathcal{L}_{\gamma}$ process, see Lemma 3.9 of [21], that we use in the following form

Lemma 4.3 (Superexponential replacement lemma for the $\mathcal{L}_{\gamma}$ process). For any $\delta>0$ and any initial probability measures $\mu, \mathbb{P}$ a.s.

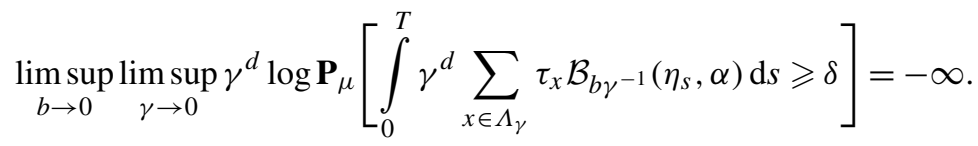

Lemma 4.4 (Superexponential replacement lemma for the $\mathcal{L}_{\gamma}^{V, v}$ process). For any $\delta>0$ and any initial probability measures $\mu, \mathbb{P}$ a.s.

$$
\underset{b \rightarrow 0}{\limsup \limsup } \gamma_{\gamma \rightarrow 0}^{d} \log \mathbf{P}_{\mu}^{V, v}\left[\int_{0}^{T} \gamma^{d} \sum_{x \in \Lambda_{\gamma}} \tau_{x} \mathcal{B}_{b \gamma^{-1}}\left(\eta_{s}, \alpha\right) \mathrm{d} s \geqslant \delta\right]=-\infty .
$$

Proof. Denote by $\mathcal{A}_{\gamma, b}^{T}$ the set $\mathcal{A}_{\gamma, b}^{T}=\left\{\int_{0}^{T} \gamma^{d} \sum_{x \in \Lambda_{\gamma}} \tau_{x} \mathcal{B}_{b \gamma^{-1}}\left(\eta_{s}, \alpha\right) \mathrm{d} s \geqslant \delta\right\}$. By Hölder inequality, for all $\varrho>1$

$$
\gamma^{d} \log \mathbf{P}_{\mu}^{V, v}\left[\mathcal{A}_{\gamma, b}^{T}\right] \leqslant \frac{\gamma^{d}}{\varrho} \log \mathbf{P}_{\mu}\left[\mathcal{A}_{\gamma, b}^{T}\right]+\gamma^{d} \frac{\varrho-1}{\varrho} \log \mathbf{E}^{\mathbf{P}_{\mu}}\left[\left(\frac{\mathrm{d} \mathbf{P}_{\mu}^{V, v}}{\mathrm{~d} \mathbf{P}_{\mu}}\right)^{\varrho /(\varrho-1)}\right] .
$$

From Lemma 4.3 it is enough to show that there exists $\varrho>1$ such that

$$
\lim _{\gamma \rightarrow 0} \gamma^{d} \frac{\varrho-1}{\varrho} \log \mathbf{E}^{\mathbf{P}_{\mu}}\left[\left(\frac{\mathrm{d} \mathbf{P}_{\mu}^{V, v}}{\mathrm{~d} \mathbf{P}_{\mu}}\right)^{\varrho /(\varrho-1)}\right] \leqslant C
$$


for some constant $C$. The Radon-Nikodym derivative, see [14] (Appendix 1, Section 7, Proposition 7.3.), is given by

$$
\frac{\mathrm{d} \mathbf{P}_{\mu}^{V, v}}{\mathrm{~d} \mathbf{P}_{\mu}}=\exp \left\{-\frac{1}{2} H_{\gamma}^{V, v}\left(\eta_{t}\right)+\frac{1}{2} H_{\gamma}^{V, v}\left(\eta_{0}\right)-\int_{0}^{t} \exp \left\{\frac{1}{2} H_{\gamma}^{V, v}\left(\eta_{s}\right)\right\}\left(\partial_{s}+\gamma^{-2} \mathcal{L}_{\gamma}\right) \exp \left\{-\frac{1}{2} H_{\gamma}^{V, v}\left(\eta_{s}\right)\right\} \mathrm{d} s\right\} .
$$

By Taylor expansion up to the second order and the elementary inequality $|R(u)| \leqslant \frac{1}{6}\left|u^{3}\right| \mathrm{e}^{|u|}$, we obtain

$$
\frac{\mathrm{d} \mathbf{P}_{\mu}^{V, v}}{\mathrm{~d} \mathbf{P}_{\mu}}=\mathcal{Z}_{T}^{\frac{V}{2}, \frac{v}{2}} \exp \left\{\gamma^{-d} \mathbf{O}_{V, v}(\gamma)\right\}
$$

where $\mathcal{Z}_{T}^{\frac{V}{2}, \frac{v}{2}}$ is the exponential martingale obtained setting $G=\frac{V}{2}$ and $g=\frac{v}{2}$ in (5.6). The $\mathrm{O}_{V, v}(\gamma)$ is bounded by $C \gamma$, where $C=C(V, v)$. Therefore,

$$
\begin{aligned}
\left(\frac{\mathrm{d} \mathbf{P}_{\mu}^{V, v}}{\mathrm{~d}_{\mu}}\right)^{\varrho /(\varrho-1)} & =\left(\mathcal{Z}_{T}^{\frac{V}{2}, \frac{v}{2}}\right)^{\varrho /(\varrho-1)} \exp \left\{\frac{\varrho}{\varrho-1} \gamma^{-d} \mathrm{O}_{V, v}(\gamma)\right\} \\
& =\mathcal{Z}_{T}^{\frac{\varrho}{\varrho-1} \frac{V}{2}, \frac{v}{2}} \times \exp \left\{\frac{\gamma^{-2 d}}{2} \frac{\varrho}{(\varrho-1)^{2}}\left\langle M^{\frac{V}{2}, \frac{v}{2}}\right\rangle_{T}\right\} \exp \left\{\frac{\varrho}{\varrho-1} \gamma^{-d} \mathrm{O}_{V, v}(\gamma)\right\} .
\end{aligned}
$$

Observe that, from the expression of $\left\langle M^{\frac{V}{2}, \frac{v}{2}}\right\rangle_{T}$, see (5.8), there exists a constant $M=M(V, v, J, T)$ such that $\gamma^{-2 d}\left\langle M^{\frac{V}{2}, \frac{v}{2}}\right\rangle_{T} \leqslant M \gamma^{-d}$. Then

$$
\begin{aligned}
\gamma^{d} \frac{\varrho-1}{\varrho} \log \mathbf{E}^{\mathbf{P}_{\mu}}\left[\left(\frac{\mathrm{d} \mathbf{P}_{\mu}^{V, v}}{\mathrm{~d} \mathbf{P}_{\mu}}\right)^{\varrho /(\varrho-1)}\right] & \leqslant \gamma^{d} \frac{\varrho-1}{\varrho} \log \mathbf{E}^{\mathbf{P}_{\mu}}\left[\mathcal{Z}_{T}^{\frac{\varrho}{\varrho-1} \frac{V}{2}, \frac{v}{2}}\right]+\frac{M}{2(\varrho-1)}+\mathrm{O}_{V, v}(\gamma) \\
& =\frac{M}{2(\varrho-1)}+\mathrm{O}_{V, v}(\gamma) .
\end{aligned}
$$

This concludes the proof of the lemma.

\subsection{Identification of the limit}

The identification of the limit is done following the same steps as in [21]. For $(\eta, \alpha) \in \mathcal{S}_{\gamma} \times \Omega_{D}$, let

$$
\begin{aligned}
& \mathbf{J}_{x, x+e}^{V, v}=C_{\gamma}^{V, v}(x, x+e ; \eta)[\eta(x)-\eta(x+e)], \\
& \mathbf{J}_{x, x+e}=C_{\gamma}(x, x+e ; \eta)[\eta(x)-\eta(x+e)]
\end{aligned}
$$

be the currents associated to the generator $\gamma^{-2} \mathcal{L}_{\gamma}^{V, v}$ and, respectively, to $\gamma^{-2} \mathcal{L}_{\gamma}$. Split $\mathbf{J}_{x, x+e}^{V, v}$ as

$$
\mathbf{J}_{x, x+e}^{V, v}=\mathbf{J}_{x, x+e}+\left[\mathbf{J}_{x, x+e}^{V, v}-\mathbf{J}_{x, x+e}\right] .
$$

From (4.11) one obtains

$$
\begin{aligned}
\mathbf{J}_{x, x+e}^{V, v}-\mathbf{J}_{x, x+e}= & \frac{\gamma}{2} C^{0}(x, x+e ; \eta)[\eta(x)-\eta(x+e)]^{2}\left(\partial_{e}^{\gamma} V\right)(t, \gamma x) \\
& +\frac{\gamma}{2} \mathbf{J}_{x, x+e}^{0}\left\{\sum_{k=1}^{d}\left(\partial_{e_{k}}^{\gamma} V\right)(t, \gamma x)\left(\nabla_{x, x+e} \Gamma_{v_{k}}\right)(\eta)\right\}+\mathrm{O}_{u}\left(\gamma^{2}\right) \mathrm{O}_{u}\left(\ell^{d}\right) .
\end{aligned}
$$

Set, for $k=1, \ldots, d, 0<a<1,0<c<1$ and $x \in \Lambda_{\gamma}$

$$
\begin{aligned}
\mathbb{V}_{k}^{\gamma, c, a}(s, x, \eta) \equiv & \mathbf{J}_{x, x+e_{k}}^{V, v}+\gamma \sum_{m=1}^{d} D_{k, m}\left(\eta^{\left[a \gamma^{-1}\right]}(x)\right)\left\{(2 c)^{-1} \tau_{x}\left(\eta^{\left[a \gamma^{-1}\right]}\left[c \gamma^{-1} e_{m}\right]-\eta^{\left[a \gamma^{-1}\right]}\left[-c \gamma^{-1} e_{m}\right]\right)\right. \\
& \left.-\chi\left(\eta^{\left[a \gamma^{-1}\right]}(x)\right)\left(\beta \partial_{e_{m}}\left(J \star \pi^{\gamma}\right)(\eta)(\gamma x)+\partial_{e_{m}} V(s, \gamma x)\right)\right\},
\end{aligned}
$$

where $D(\cdot)$ and $\chi(\cdot)$ are defined in (2.19) and (2.20). Next theorem is the main step in the proof of Theorem 4.1. 
Theorem 4.5. Let $d \geqslant 3$. For $G$ in $\mathcal{C}^{1,2}([0, T] \times \Lambda), t \in[0, T]$ and $\delta>0$, $\mathbb{P}$ a.s.

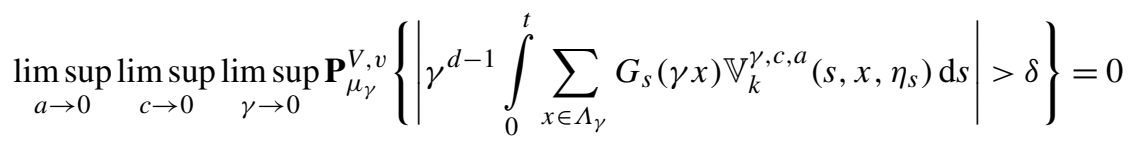

for $k=1, \ldots, d$.

By standard summation by parts, Theorem 4.5 allows to conclude the proof of Theorem 4.1. Details can be found in section 7.1. of [14] and [21].

Proof of Theorem 4.5. By standard stochastic calculus it can be proven that for any $g=\left(g_{1}, \ldots, g_{d}\right) \in \mathbb{G}^{d}$, for $1 \leqslant k \leqslant d$ and $t \in[0, T]$

$$
\limsup _{\gamma \rightarrow 0} \mathbf{E}_{\mu_{\gamma}}^{\mathbf{P}^{V, v}}\left[\left|\int_{0}^{t} \mathrm{~d} s\left(\gamma^{d-1} \sum_{x \in \Lambda_{\gamma}} G_{s}(\gamma x) \tau_{x} \mathcal{L}_{\gamma}^{V, v} g_{k}\left(\eta_{s}, \alpha\right)\right)\right|\right]=0
$$

for all real smooth, bounded functions $G_{s}(u)=G(s, u)$ defined on $\mathbb{R}_{+} \times \Lambda_{\gamma}$. Namely

$$
\begin{aligned}
& \int_{0}^{t} \mathrm{~d} s\left(\gamma^{d-1} \sum_{x \in \Lambda_{\gamma}} G_{s}(\gamma x) \tau_{x} \mathcal{L}_{\gamma}^{V, v} g_{k}\left(\eta_{s}, \alpha\right)\right) \\
& \quad=\gamma^{d+1} \sum_{x \in \Lambda_{\gamma}}\left[G_{t}(\gamma x) \tau_{x} g_{k}\left(\eta_{t}, \alpha\right)-G_{0}(\gamma x) \tau_{x} g_{k}\left(\eta_{0}, \alpha\right)\right]+\gamma \widetilde{M}_{\gamma}^{G}(t)+E_{\gamma}^{G}(t),
\end{aligned}
$$

where $\left\{\tilde{M}_{\gamma}^{G}(t)\right\}_{0 \leqslant t \leqslant T}$ is a $\mathbf{P}_{\mu_{\gamma}}^{V, v}$ martingale with respect to the natural filtration and has quadratic variation of order $\mathrm{O}_{u}\left(\gamma^{d}\right)$. The first term in the second line of (4.28) is of order $\gamma$ and the last term $E_{\gamma}^{G}(t)$ is the error term coming from ignoring the action of the generator on the first variable of $g_{k}$. One has that $\sup _{t \in[0, T]}\left|E_{\gamma}^{G}(t)\right|$ tends to zero in probability and $\mathbb{P}$ a.s as $\gamma \rightarrow 0$ and the diameter of the support of $g_{k}$ to $\infty$, see [14], Chapter VII. Then (4.27) follows. Taking in account (4.27), we prove (4.26) showing that for $t \in[0, T]$ and any $\delta>0$

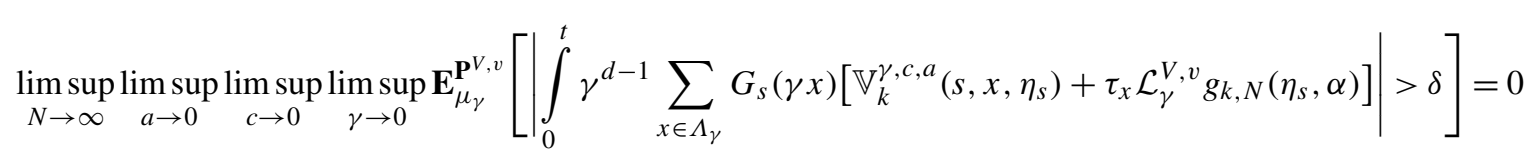

for all $1 \leqslant k \leqslant d$ where the sequence $\left(g^{N}=\left(g_{1, N}, \ldots, g_{d, N}\right)\right)_{N \geqslant 1}$ is a convenient chosen sequence of local functions. Let $V_{\rho}(\cdot, \cdot), 0 \leqslant \rho \leqslant 1$, be the quantity defined in Section 8, see (A.3) and below of it. Since from [8], Theorem 7.22,

$$
\inf _{\left(g_{1}, \ldots, g_{d}\right) \in \mathbb{G}^{d}} \limsup _{n \uparrow \infty} \sup _{0 \leqslant \rho \leqslant 1} \sum_{k=1}^{d} V_{\rho}\left(\mathbf{J}_{0, e_{k}}^{0}+\sum_{m=1}^{d} D_{k, m}(\rho) \frac{\psi_{n, n}^{e_{m}}}{n}+\mathcal{L}^{0} g_{k}(\eta, \alpha)\right)=0,
$$

we take $\left(g^{N}\right)$ so that for any integer $N \geqslant 1$

$$
\limsup _{n \uparrow \infty} \sup _{0 \leqslant \rho \leqslant 1} \sum_{k=1}^{d} V_{\rho}\left(\mathbf{J}_{0, e_{k}}^{0}+\sum_{m=1}^{d} D_{k, m}(\rho) \frac{\psi_{n, n}^{e_{m}}}{n}+\mathcal{L}^{0} g_{k, N}\right) \leqslant \frac{1}{N} .
$$

Next, for $0 \leqslant s \leqslant T$ and $\eta \in \mathcal{S}_{\gamma}$ split

$$
\mathbb{V}_{k}^{\gamma, c, a}(s, x, \eta)+\tau_{x} \mathcal{L}_{\gamma, s}^{V, v} g_{k, N}(\eta, \alpha)=\tau_{x} Y_{k}^{\gamma, c, a, N}(\eta, \alpha)+Z_{k}^{\gamma, c, a, N}(s, x, \eta, \alpha),
$$


where

$$
\begin{aligned}
& Y_{k}^{\gamma, c, a, N}(\eta, \alpha)= \mathbf{J}_{0, e_{k}}+\mathcal{L}_{\gamma} g_{k, N}+\gamma \sum_{m=1}^{d} D_{k, m}\left(\eta^{\left[a \gamma^{-1}\right]}(0)\right)\left\{(2 c)^{-1}\left(\eta^{\left[a \gamma^{-1}\right]}\left[c \gamma^{-1} e_{m}\right]-\eta^{\left[a \gamma^{-1}\right]}\left[-c \gamma^{-1} e_{m}\right]\right)\right\} \\
&-\gamma \sum_{m=1}^{d} D_{k, m}\left(\eta^{\left[a \gamma^{-1}\right]}(0)\right) \chi\left(\eta^{\left[a \gamma^{-1}\right]}(0)\right)\left\{\beta\left(\partial_{e_{m}} J \star \pi^{\gamma}(\eta)(0)\right)\right\}, \\
& Z_{k}^{\gamma, c, a, N}(s, x, \eta, \alpha)=\left(\mathbf{J}_{x, x+e_{k}}^{V, v}-\mathbf{J}_{x, x+e_{k}}\right)+\tau_{x}\left(\mathcal{L}_{\gamma}^{V, v}-\mathcal{L}_{\gamma}\right) g_{k, N} \\
&-\gamma \sum_{m=1}^{d} D_{k, m}\left(\eta^{\left[a \gamma^{-1}\right]}(x)\right) \chi\left(\eta^{\left[a \gamma^{-1}\right]}(x)\right)\left\{\partial_{e_{m}} V(s, \gamma x)\right\} .
\end{aligned}
$$

To conclude the proof of the theorem, taking in account (4.29), it is enough to prove the following two lemmas:

Lemma 4.6. For almost all $\alpha \in \Omega_{D}$, for any function $G \in \mathcal{C}^{1,2}([0, T] \times \Lambda)$, for any $\delta>0$

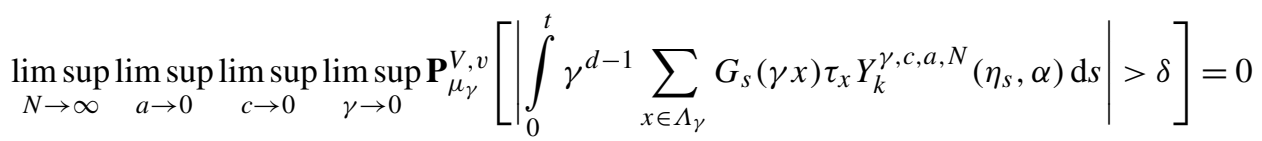

for $k=1, \ldots, d$ and any $t \in[0, T]$.

Proof. Since Lemma 4.4 it will be enough to show (4.34) with $\mathbf{P}_{\mu_{\gamma}}^{V, v}$ replaced by $\mathbf{P}_{\mu_{\gamma}}$. This has been shown in Theorem 4.1 of [21].

Lemma 4.7. For $G \in \mathcal{C}^{1,2}([0, T] \times \Lambda)$, for $\delta>0, \mathbb{P}$ a.s.

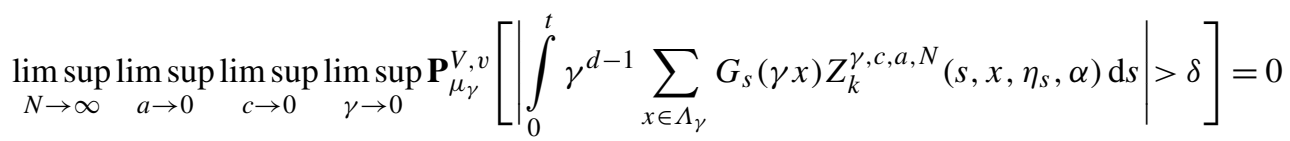

for $k=1, \ldots, d$ and any $t \in[0, T]$.

Proof. We start analyzing the first addend of $Z_{k}^{\gamma, c, a, \delta}$, the difference $\mathbf{J}_{x, x+e_{k}}^{V, v}-\mathbf{J}_{x, x+e_{k}}$. Set

$$
\begin{aligned}
& F_{1}^{k}(\eta, \alpha)=\frac{1}{2} C^{0}\left(0, e_{k} ; \eta\right)\left(\eta\left(e_{k}\right)-\eta(0)\right)^{2}, \\
& R_{1}^{k, m}(\eta, \alpha)=\frac{1}{2} \mathbf{J}_{0, e_{k}}^{0}\left\{\left(\nabla_{0, e_{k}} \Gamma_{v_{m}}\right)(\eta)\right\}
\end{aligned}
$$

for $1 \leqslant k, m \leqslant d$. By (4.24) we obtain

$$
\begin{aligned}
& \gamma^{d-1} \sum_{x \in \Lambda_{\gamma}} G_{s}(\gamma x)\left[\mathbf{J}_{x, x+e_{k}}^{V, v}-\mathbf{J}_{x, x+e_{k}}\right]=\gamma^{d} \sum_{x \in \Lambda_{\gamma}} G_{s}(\gamma x) \partial_{e_{k}} V(s, \gamma x) \tau_{x} F_{1}^{k}(\eta, \alpha) \\
& +\gamma^{d} \sum_{m=1}^{d} \sum_{x \in \Lambda_{\gamma}} G_{s}(\gamma x)\left(\partial_{e_{m}} V\right)(s, \gamma x) \tau_{x} R_{1}^{k, m}(\eta, \alpha)+\mathrm{O}_{u}(\gamma) \mathrm{O}_{u}\left(\ell^{d}\right) .
\end{aligned}
$$

Denote for $t \in[0, T]$ 


$$
\begin{aligned}
& \mathcal{A}_{a, \gamma}\left(\left\{\eta_{s}\right\}_{0 \leqslant s \leqslant t}\right)=\int_{0}^{t} \mathrm{~d} s\left\{\gamma^{d-1} \sum_{x \in \Lambda_{\gamma}} G_{s}(\gamma x)\left[\mathbf{J}_{x, x+e_{k}}^{V, v}\left(\eta_{s}\right)-\mathbf{J}_{x, x+e_{k}}\left(\eta_{s}\right)\right]\right\} \\
& \quad-\int_{0}^{t} \mathrm{~d} s\left\{\gamma^{d} \sum_{m=1}^{d} \sum_{x \in \Lambda_{\gamma}} G_{s}(\gamma x)\left(\partial_{e_{m}} V\right)(s, \gamma x)\left(\frac{1}{\left(2 a \gamma^{-1}+1\right)^{d}} \sum_{|y| \leqslant a \gamma^{-1}} \tau_{x+y} R_{1}^{k, m}\left(\eta_{s}, \alpha\right)\right)\right\} \\
& -\int_{0}^{t} \mathrm{~d} s\left\{\gamma^{d} \sum_{x \in \Lambda_{\gamma}} G_{s}(\gamma x)\left(\partial_{e_{k}} V\right)(s, \gamma x)\left(\frac{1}{\left(2 a \gamma^{-1}+1\right)^{d}} \sum_{|y| \leqslant a \gamma^{-1}} \tau_{x+y} F_{1}^{k}\left(\eta_{s}, \alpha\right)\right)\right\} .
\end{aligned}
$$

Since the functions $R_{1}^{k, m}$ and $F_{1}^{k}, 1 \leqslant k, m \leqslant d$, are bounded, by the smoothness of $G$ and $V$, a spatial summation by parts and Taylor expansion permit, to show that, for $t \in[0, T]$ and $\alpha \in \Omega_{D}$

$$
\lim _{a \rightarrow 0} \limsup _{\gamma \rightarrow 0} \sup _{\left\{\eta_{s}\right\}_{s} \geqslant 0}\left|\mathcal{A}_{a, \gamma}\left(\left\{\eta_{s}\right\}_{0 \leqslant s \leqslant t}\right)\right|=0 .
$$

Recalling (4.16), denote

$$
\widetilde{F}^{k}(\rho)=\mathbb{E}\left[\mathbf{E}^{\mu^{\alpha, \lambda_{0}(\rho)}}\left(F_{1}^{k}\right)\right] \text { and }{\widetilde{R_{1}}}^{k, m}(\rho)=\mathbb{E}\left[\mathbf{E}^{\mu^{\alpha, \lambda_{0}(\rho)}}\left(R_{1}^{k, m}\right)\right],
$$

and for $0 \leqslant s \leqslant T, u \in \Lambda$ and $(\eta, \alpha) \in \mathcal{S}_{\gamma} \times \Omega_{D}$

$$
\mathcal{B}_{1}^{k, a}(s, u, \eta, \alpha)=\gamma^{-1}\left(\mathbf{J}_{0, e_{k}}^{V, v}-\mathbf{J}_{0, e_{k}}\right)-\left\{\left(\partial_{e_{k}} V\right)(s, u){\widetilde{F_{1}}}^{k}\left(\eta^{\left[a \gamma^{-1}\right]}(0)\right)+\sum_{m=1}^{d}\left(\partial_{e_{m}} V\right)(s, u){\widetilde{R_{1}}}^{k, m}\left(\eta^{\left[a \gamma^{-1}\right]}(0)\right)\right\} .
$$

The (4.37) together with Lemma 4.4 applied to the local functions $F_{1}^{k}$ and $R_{1}^{k, m}$, implies that for $t \in[0, T]$ and $\delta>0$, $\mathbb{P}$ a.s.

$$
\lim _{a \rightarrow 0} \lim _{\gamma \rightarrow 0} \mathbf{P}_{\mu_{\gamma}}^{V, v}\left[\left|\gamma^{d} \sum_{x \in \Lambda_{\gamma}} \int_{0}^{t} G_{s}(\gamma x) \tau_{x} \mathcal{B}_{1}^{k, a}\left(s, \gamma x, \eta_{s}, \alpha\right) \mathrm{d} s\right|>\delta\right]=0 .
$$

Next we consider the second term of $Z_{k}^{\gamma, c, a, N}$, the difference $\left(\mathcal{L}_{\gamma}^{V, v}-\mathcal{L}_{\gamma}\right) g_{k, N}$, and repeat the same steps used for the first term. By Lemma 4.2 we have

$$
\begin{aligned}
& \left(\mathcal{L}_{\gamma}^{V, v}-\mathcal{L}_{\gamma}\right) g_{k, N}(\eta, \alpha)=\frac{\gamma}{2} \sum_{m=1}^{d} \sum_{y \in \Lambda_{\gamma}} \mathbf{J}_{y, y+e_{m}}^{0}\left(\partial_{e_{m}} V\right)(s, \gamma y)\left[\nabla_{y, y+e_{m}} g_{k, N}\right] \\
& \quad+\frac{\gamma}{2} \sum_{m=1}^{d} \sum_{y \in \Lambda_{\gamma}} C^{0}\left(y, y+e_{m} ; \eta\right)\left\{\sum_{j=1}^{d}\left(\partial_{e_{j}} V\right)(s, \gamma y) \nabla_{y, y+e_{m}}\left(\Gamma_{v_{j}}(\eta)\right)\right\}\left[\nabla_{y, y+e_{m}} g_{k, N}\right]+\mathrm{O}_{u}\left(\gamma^{2-d}\right) \mathrm{O}_{u}\left(\ell^{d}\right) .
\end{aligned}
$$

Denote

$$
\begin{aligned}
& F_{2}^{k, m, N}(\eta, \alpha)=\frac{1}{2} \sum_{y \in \Lambda_{\gamma}} \mathbf{J}_{y, y+e_{m}}^{0}\left[\nabla_{y, y+e_{m}} g_{k, N}\right], \\
& R_{2}^{k, j, m, N}(\eta, \alpha)=\frac{1}{2} \sum_{y \in \Lambda_{\gamma}} C^{0}\left(y, y+e_{m} ; \eta\right)\left(\nabla_{y, y+e_{m}} \Gamma_{v_{j}}(\eta)\right)\left[\nabla_{y, y+e_{m}} g_{k, N}\right] .
\end{aligned}
$$

We obtain that

$$
\begin{aligned}
& \gamma^{d-1} \sum_{x \in \Lambda_{\gamma}} G_{s}(\gamma x) \tau_{x}\left(\mathcal{L}_{\gamma}^{V, v}-\mathcal{L}_{\gamma}\right) g_{k, N}(\eta, \alpha)=\gamma^{d} \sum_{m=1}^{d} \sum_{x \in \Lambda_{\gamma}} G_{s}(\gamma x)\left(\partial_{e_{m}} V\right)(s, \gamma x) \tau_{x} F_{2}^{k, m, N}(\eta, \alpha) \\
& +\gamma^{d} \sum_{m=1}^{d} \sum_{x \in \Lambda_{\gamma}} G_{s}(\gamma x) \sum_{j=1}^{d}\left(\partial_{e_{j}} V\right)(s, \gamma x) \tau_{x} R_{2}^{k, j, m, N}(\eta, \alpha)+\mathrm{O}_{u}(\gamma) \mathrm{O}_{u}\left(\ell^{d}\right) .
\end{aligned}
$$


Denoting

$$
\mathcal{R}_{k, N}^{j}(\eta, \alpha)=\sum_{m=1}^{d} R_{2}^{k, j, m, N}(\eta, \alpha),
$$

we write (4.42) as

$$
\begin{aligned}
& \gamma^{d-1} \sum_{x \in \Lambda_{\gamma}} G_{s}(\gamma x) \tau_{x}\left(\mathcal{L}_{\gamma}^{V, v}-\mathcal{L}_{\gamma}\right) g_{k, N}(\eta, \alpha)=\gamma^{d} \sum_{m=1}^{d} \sum_{x \in \Lambda_{\gamma}} G_{s}(\gamma x)\left(\partial_{e_{m}} V\right)(s, \gamma x) \tau_{x} F_{2}^{k, m, N}(\eta, \alpha) \\
& +\gamma^{d} \sum_{m=1}^{d} \sum_{x \in \Lambda_{\gamma}} G_{s}(\gamma x)\left(\partial_{e_{m}} V\right)(s, \gamma x) \tau_{x} \mathcal{R}_{k, N}^{m}(\eta, \alpha)+\mathrm{O}_{u}(\gamma) \mathrm{O}_{u}\left(\ell^{d}\right) .
\end{aligned}
$$

Next, we exploit that $g_{k, N}$ and $v_{j}$ are local and bounded functions. For sake of simplicity, we suppose that $g_{k, N}$ has support in the cube centered at the origin of side $\ell$ and denote for $u \in \Lambda,(\eta, \alpha) \in \mathcal{S}_{\gamma} \times \Omega_{D}$ and $s \in[0, T]$

$$
\mathcal{B}_{2}^{k, a, N}(s, u, \eta, \alpha)=\gamma^{-1}\left(\mathcal{L}_{\gamma}^{V, v}-\mathcal{L}_{\gamma}\right) g_{k, N}-\sum_{m=1}^{d}\left(\partial_{e_{m}} V\right)(s, u)\left\{\widetilde{F}_{2}^{k, m, N}\left(\eta^{\left[a \gamma^{-1}\right]}(0)\right)+\widetilde{\mathcal{R}}_{k, N}^{m}\left(\eta^{\left[a \gamma^{-1}\right]}(0)\right)\right\} .
$$

By using Lemma 4.3 we obtain that, for any fixed $N \geqslant 1$, for any $\delta>0$ and $t \in[0, T]$, for almost all $\alpha \in \Omega_{D}$

$$
\lim _{a \rightarrow 0} \lim _{\gamma \rightarrow 0} \mathbf{P}_{\mu_{\gamma}}^{V, v}\left[\left|\gamma^{d} \sum_{x \in \Lambda_{\gamma}} \int_{0}^{t} G_{s}(\gamma x) \tau_{x} \mathcal{B}_{2}^{k, a, N}\left(s, \gamma x, \eta_{s}, \alpha\right) \mathrm{d} s\right|>\delta\right]=0 .
$$

We conclude the proof by collecting the estimates (4.39), (4.44) and using Lemma 4.8 below.

Lemma 4.8. For $1 \leqslant k, m \leqslant d$,

$$
\begin{aligned}
& \lim _{N \rightarrow \infty} \sup _{0 \leqslant \rho \leqslant 1}\left|\delta_{k, m} \widetilde{F}_{1}{ }^{k}(\rho)+\widetilde{F}_{2}{ }^{k, m, N}(\rho)-\chi(\rho) D_{k, m}(\rho)\right|=0, \\
& \lim _{N \rightarrow \infty} \sup _{0 \leqslant \rho \leqslant 1}\left|\delta_{k, m} \widetilde{F}_{1}^{k}(\rho)+\widetilde{F}_{2}{ }^{k, m, N}(\rho)+\widetilde{R}_{1}^{k, m}(\rho)+\widetilde{\mathcal{R}}_{k, N}^{m}(\rho)-\chi(\rho) D_{k, m}(\rho)\right|=0 .
\end{aligned}
$$

Proof. We start proving (4.46). From (4.36), reversibility (2.10) and (A.7) we obtain for $\rho \in[0,1]$

$$
\begin{aligned}
\widetilde{R}_{1}^{k, m}(\rho) & =\frac{1}{2} \mathbb{E}\left[\mathbf{E}^{\mu^{\alpha, \lambda_{0}(\rho)}}\left(\Phi\left(\nabla_{0, e_{k}} H_{0}^{\alpha}\right)\left(\eta(0)-\eta\left(e_{k}\right)\right)\left\{\left(\nabla_{0, e_{k}} \Gamma_{v_{m}}\right)(\eta)\right\}\right)\right] \\
& =\mathbb{E}\left[\mathbf{E}^{\mu^{\alpha, \lambda_{0}(\rho)}}\left(\Phi\left(\nabla_{0, e_{k}} H_{0}^{\alpha}\right)\left(\eta\left(e_{k}\right)-\eta(0)\right) \Gamma_{v_{m}}(\eta)\right)\right] \\
& =-\sum_{z} \mathbb{E}\left[\mathbf{E}^{\mu^{\alpha, \lambda_{0}(\rho)}}\left(\mathbf{J}_{0, e_{k}}^{0} \tau_{z} v_{m}(\eta, \alpha)\right)\right] \\
& =-\left(\mathbf{J}_{0, e_{k}}^{0}, v_{m}\right)_{\rho, 0}=V_{\rho}\left(\mathbf{J}_{0, e_{k}}^{0}, \mathcal{L}^{0} v_{m}\right),
\end{aligned}
$$

where $(\cdot, \cdot)_{\rho, 0}$ is defined in (A.6) and $\Gamma_{v_{m}}$ in (4.9). From (4.41) we obtain again by reversibility that

$$
{\widetilde{R_{2}}}^{k, j, m, N}(\rho)=-\sum_{y \in \Lambda_{\gamma}} \mathbb{E}\left[\mathbf{E}^{\mu^{\alpha, \lambda_{0}(\rho)}}\left[\Phi\left(\nabla_{y, y+e_{m}} H_{0}^{\alpha}\right)\left(\nabla_{y, y+e_{m}} \Gamma_{v_{j}}\right)(\eta) g_{k, N}\right]\right] .
$$

Since (4.43) and (4.9)

$$
\begin{aligned}
\widetilde{\mathcal{R}}_{k, N}^{j}(\rho) & =-\sum_{z \in \Lambda_{\gamma}} \mathbb{E}\left[\mathbf{E}^{\mu^{\alpha, \lambda_{0}(\rho)}}\left[\tau_{z}\left(\mathcal{L}^{0} v_{j}\right) g_{k, N}\right]\right] \\
& =-\left(\mathcal{L}^{0} v_{j}, g_{k, N}\right)_{\rho, 0}=V_{\rho}\left(\mathcal{L}^{0} v_{j}, \mathcal{L}^{0} g_{k, N}\right) .
\end{aligned}
$$


On the other hand, from (A.7), (A.8) and reversibility, the same computations done for (4.38) and (4.47) yield, for $\rho \in[0,1]$

$$
\delta_{k, m} \widetilde{F}_{1}^{k}(\rho)=V_{\rho}\left(\mathbf{J}_{0, e_{k}}^{0}, \mathbf{J}_{0, e_{m}}^{0}\right)
$$

and

$$
\widetilde{F}_{2}^{k, m, N}(\rho)=V_{\rho}\left(\mathbf{J}_{0, e_{m}}^{0}, \mathcal{L}^{0} g_{k, N}\right)
$$

Taking in account property (P) defined in Appendix A, after the formula (A.4), applying (A.8) we obtain by Remark 7.20 in [8], that

$$
\begin{aligned}
& \sup _{0 \leqslant \rho \leqslant 1}\left|\delta_{k, m} \widetilde{F}_{1}^{k}(\rho)+{\widetilde{F_{2}}}^{k, m, N}(\rho)+{\widetilde{R_{1}}}^{k, m}(\rho)+\widetilde{\mathcal{R}}_{k, N}^{m}(\rho)-\chi(\rho) D_{k, m}(\rho)\right| \\
& \quad=\limsup _{n \uparrow \infty} \sup _{0 \leqslant \rho \leqslant 1}\left|V_{\rho}\left(\mathbf{J}_{0, e_{m}}^{0}+\mathcal{L}^{0} v_{m}, \mathbf{J}_{0, e_{k}}^{0}+\sum_{\ell=1}^{d} D_{k, \ell}(\rho) \frac{\psi_{n, n}^{e_{\ell}}}{n}+\mathcal{L}^{0} g_{k, N}\right)\right| .
\end{aligned}
$$

By Schwartz inequality, the right-hand side of the last equality is bounded by

$$
\limsup _{n \uparrow \infty} \sup _{0 \leqslant \rho \leqslant 1}\left\{V_{\rho}^{1 / 2}\left(\mathbf{J}_{0, e_{m}}^{0}+\mathcal{L}^{0} v_{m}\right) V_{\rho}^{1 / 2}\left(\mathbf{J}_{0, e_{k}}^{0}+\sum_{\ell=1}^{d} D_{k, \ell}(\rho) \frac{\psi_{n, n}^{e_{\ell}}}{n}+\mathcal{L}^{0} g_{k, N}\right)\right\}
$$

which is bounded, see Theorem 7.22 of [8], ${ }^{5}$ by $C / \sqrt{N}$ for some positive constant $C$. To conclude the proof of the lemma it remains to let $N \uparrow \infty$. Similar considerations apply to (4.45) obtaining

$$
\begin{aligned}
& \sup _{0 \leqslant \rho \leqslant 1}\left|\delta_{k, m} \widetilde{F}_{1}^{k}(\rho)+\widetilde{F}_{2}^{k, m, N}(\rho)-\chi(\rho) D_{k, m}(\rho)\right| \\
& \quad=\limsup _{n \uparrow \infty} \sup _{0 \leqslant \rho \leqslant 1}\left|V_{\rho}\left(\mathbf{J}_{0, e_{m}}^{0}, \mathbf{J}_{0, e_{k}}^{0}+\sum_{\ell=1}^{d} D_{k, \ell}(\rho) \frac{\psi_{n, n}^{e_{\ell}}}{n}+\mathcal{L}^{0} g_{k, N}\right)\right| .
\end{aligned}
$$

We conclude the proof applying, as for (4.50), Schwartz inequality, Theorem 7.33 of [8] and letting $N \uparrow \infty$.

\section{Upper bound}

Recall from Section 2 that for $\rho_{0}: \Lambda \rightarrow(0,1)$, the measure $\nu_{\gamma}^{\rho_{0}}$ stands for the Bernoulli product measure on $\mathcal{S}_{\gamma}$ with $\nu_{\gamma}^{\rho_{0}}\{\eta(x)=1\}=\rho_{0}(\gamma x)$. Further $\mathbf{P}_{v_{\gamma}}{ }_{\rho_{0}}$ (resp. $\left.Q_{v_{\gamma}^{\rho_{0}}}\right)$ denotes the law of the process $\left(\eta_{t}\right)$ (resp. $\left.\left(\pi_{t}\right)\right)$ starting from $v_{\gamma}^{\rho_{0}}$. Following the usual method, to exhibit an upper bound, we need to find a family of $\mathbf{P}_{\nu_{\gamma}} \rho_{0}$-mean-one positive martingales that can be expressed as function of the empirical density. For almost all disorder $\alpha$, when $\gamma \rightarrow 0$, these martingales will then produce a family $\left(\widehat{F}_{a, c}^{G, m}\right)_{0<a<c<1}$, (defined next in (5.2)), of functionals on the space $D\left([0, T], \mathcal{M}_{1}(\Lambda)\right.$ ). For $a>0$, denote by $\iota_{a}$ the approximation of the identity defined by

$$
\iota_{a}(u)=\frac{1}{(2 a)^{d}} \mathbb{1}_{\left\{[-a, a]^{d}\right\}}(u) .
$$

Let $G(\cdot, \cdot) \in C^{1,2}([0, T] \times \Lambda), m: \Lambda \rightarrow(0,1)$ continuous and $\mu \in D\left([0, T], \mathcal{M}_{1}(\Lambda)\right)$. For $0<a<c<1$, define the following functional

\footnotetext{
5 Since we are assuming that $D(\rho)$ can be continuously extended in $[0,1]$, the $\rho$ in the sup can vary in $[0,1]$.
} 


$$
\begin{aligned}
\widehat{F}_{a, c}^{G, m}(\mu(\cdot, \cdot))= & f_{0}^{m}\left(\mu_{0}\right)+\left\langle G_{T}, \mu_{T}\right\rangle-\left\langle G_{0}, \mu_{0}\right\rangle-\int_{0}^{T}\left\langle\partial_{s} G_{s}, \mu_{s}\right\rangle \mathrm{d} s \\
& +\sum_{1 \leqslant k, j \leqslant d} \int_{0}^{T} \mathrm{~d} s \int_{\Lambda} \mathrm{d} r\left(\partial_{e_{k}} G_{s}\right)(r)\left\{D_{k, j}\left(\left(\mu_{s} \star \iota_{a}\right)(r)\right)\right. \\
& \left.\times\left\{(2 c)^{-1}\left[\left(\mu_{s} \star \iota_{a}\right)\left(r+c e_{j}\right)-\left(\mu_{s} \star \iota_{a}\right)\left(r-c e_{j}\right)\right]-\beta \chi\left(\left(\mu_{s} \star \iota_{a}\right)(r)\right)\left(\partial_{e_{j}} J \star \mu_{s}(r)\right)\right\}\right\} \\
& -\frac{1}{2} \sum_{1 \leqslant k, j \leqslant d} \int_{0}^{T} \mathrm{~d} s \int_{\Lambda} \mathrm{d} r\left(\partial_{e_{k}} G_{s}\right)(r) \sigma_{k, j}\left(\left(\mu_{s} \star \iota_{a}\right)(r)\right)\left(\partial_{e_{j}} G_{s}\right)(r),
\end{aligned}
$$

where $G_{s}(r) \equiv G(s, r)$ and $f_{0}^{m}\left(\mu_{0}\right)$ is given by

$$
f_{0}^{m}\left(\mu_{0}\right)=\left\langle\log \left(\frac{m(\cdot)\left(1-\rho_{0}(\cdot)\right)}{\rho_{0}(\cdot)(1-m(\cdot))}\right), \mu_{0}\right\rangle+\int_{\Lambda} \log \left(\frac{1-m(r)}{1-\rho_{0}(r)}\right) \mathrm{d} r .
$$

The proof of the upper bound for the compact sets follows from the next three lemmas.

Lemma 5.1. For $G(\cdot, \cdot) \in C^{1,2}([0, T] \times \Lambda)$ and $m: \Lambda \rightarrow(0,1)$ continuous, $\mathbb{P}$ a.s. we have

$$
\limsup _{c \rightarrow 0} \limsup _{a \rightarrow 0} \limsup _{\gamma \rightarrow 0} \gamma^{d} \log \mathbf{E}^{Q_{v_{\gamma} \rho_{0}}}\left[\exp \left\{\gamma^{-d} \widehat{F}_{a, c}^{G, m}(\mu(\cdot, \cdot))\right\}\right] \leqslant 0 .
$$

The proof is given in Subsection 5.1.

Lemma 5.2. For all $\mu \in D\left([0, T], \mathcal{M}_{1}(\Lambda)\right)$, if $\mathcal{I}_{0}(\mu(\cdot, \cdot))<+\infty$, see (2.31), then $\mathbb{P}$ a.s.

$$
\mathcal{I}(\mu(\cdot, \cdot)) \leqslant \limsup _{c \rightarrow 0} \limsup _{a \rightarrow 0} \sup _{G \in C^{1,2}([0, T] \times \Lambda)} \sup _{\substack{m \in C^{0}(\Lambda) \\ 0<m<1}} \widehat{F}_{a, c}^{G, m}(\mu(\cdot, \cdot)) .
$$

The proof of this lemma is similar to the one given in Lemma 2.2. of [25]. We will outline the proof at the end of Subsection 5.2.

Lemma 5.3. If $\mathcal{I}_{0}(\mu(\cdot, \cdot))=\infty$ on a compact set $K$ of $D\left([0, T], \mathcal{M}_{1}(\Lambda)\right)$ then $\mathbb{P}$ a.s.

$$
\limsup _{\gamma \rightarrow 0} \gamma^{d} \log Q_{\nu_{\gamma}}(K)=-\infty \text {. }
$$

The Lemma 5.3 is proved in Subsection 5.2. From these lemmas and the lower semicontinuity of the functional

$$
\sup _{G \in C^{1,2}([0, T] \times \Lambda)} \sup _{\substack{m \in C^{0}(\Lambda) \\ 0<m<1}} \widehat{F}_{a, c}^{G, m}(\mu(\cdot, \cdot))
$$

we obtain by standard arguments (see [25] and [14]) the upper bound for the compact sets. To extend the upper bound to an arbitrary closed set, it is enough to prove the exponential tightness (cf. [14]): there exists a sequence of compact sets $\left\{\mathcal{K}_{n}\right\} \in D\left([0, T], \mathcal{M}_{1}(\Lambda)\right)$ such that, $\mathbb{P}$ a.s.

$$
\limsup _{\gamma \rightarrow 0} \gamma^{d} \log Q_{\nu_{\gamma}^{\rho_{0}}}\left(\mathcal{K}_{n}^{c}\right) \leqslant-n .
$$

This property is proved in Subsection 5.2.

\subsection{Proof of Lemma 5.1}

We first introduce the exponential martingales from which the functionals $\widehat{F}_{a, c}^{G, m}$ follow. For $1 \leqslant k \leqslant d$, let $g_{k}^{\ell}$ be a bounded function, smooth in the first variable $g_{k}^{\ell}:[0,1] \times \mathcal{S} \times \Omega_{D} \rightarrow \mathbb{R}^{d}$ with $g_{k}^{\ell}\left(\eta^{\ell}(0), \cdot, \cdot\right) \in \mathbb{G}$. The function $g_{k}^{\ell}$ 
depends only on the particles in a box of size $\ell$ centered at the origin. To keep notation light we denote it shortly by $g_{k}^{\ell}(\eta, \alpha)$ and we denote by $g$ the vector $g=\left(g_{1}^{\ell}, \ldots, g_{d}^{\ell}\right)$. Define

$$
\begin{aligned}
& \xi^{\gamma}(t)=\gamma^{d+1} \sum_{z}\left(\left(\nabla^{\gamma} G_{t}\right)(\gamma z) \cdot \tau_{z} g(\eta, \alpha)\right) \equiv \sum_{k=1}^{d} \xi_{k}^{\gamma}(t), \\
& \xi_{k}^{\gamma}(t)=\gamma^{d+1} \sum_{z}\left(\partial_{e_{k}}^{\gamma} G_{t}\right)(\gamma z) \tau_{z} g_{k}^{\ell}(\eta, \alpha), \quad 1 \leqslant k \leqslant d,
\end{aligned}
$$

where, recall, for $u, v \in \mathbb{R}^{d},(u \cdot v)$ stands for the canonical product scalar of $u$ and $v$ in $\mathbb{R}^{d}$. For any $\alpha \in \Omega_{D}$ and a probability measure $\mu_{\gamma}$ in $\mathcal{S}_{\gamma}$, consider the $\mathbf{P}_{\mu_{\gamma}}$ martingales with respect to the natural filtration associated with $\left\{\eta_{t}\right\}_{t \geqslant 0}, \mathbf{M}_{t}^{G, g} \equiv \mathbf{M}_{t}^{G, g, \gamma, \beta, \alpha}$ and $\mathbf{N}_{t}^{G} \equiv \mathbf{N}_{t}^{G, g, \gamma, \beta, \alpha}, t \geqslant 0$, defined by

$$
\begin{aligned}
& \mathbf{M}_{t}^{G, g}=\left(\left\langle G_{t}, \pi_{t}^{\gamma}\right\rangle+\xi^{\gamma}(t)\right)-\left(\left\langle G_{0}, \pi_{0}^{\gamma}\right\rangle+\xi^{\gamma}(0)\right)-\int_{0}^{t}\left(\partial_{s}+\gamma^{-2} \mathcal{L}_{\gamma}\right)\left(\left\langle G_{s}, \pi_{s}^{\gamma}\right\rangle+\xi^{\gamma}(s)\right) \mathrm{d} s, \\
& \mathbf{N}_{t}^{G, g}=\left(\mathbf{M}_{t}^{G, g}\right)^{2}-\left\langle\mathbf{M}^{G, g}\right\rangle_{t},
\end{aligned}
$$

where $\pi_{s}^{\gamma}$ is the empirical measure at time $s$, see (2.24), and the quadratic variation of $\mathbf{M}^{G, g}$ is given by

$$
\left\langle\mathbf{M}^{G, g}\right\rangle_{t}=\gamma^{-2} \int_{0}^{t}\left\{\mathcal{L}_{\gamma}\left(\left\langle\pi_{s}^{\gamma}, G_{s}\right\rangle+\xi^{\gamma}(s)\right)^{2}-2\left(\left\langle\pi_{s}^{\gamma}, G_{s}\right\rangle+\xi^{\gamma}(s)\right) \mathcal{L}_{\gamma}\left(\left\langle\pi_{s}^{\gamma}, G_{s}\right\rangle+\xi^{\gamma}(s)\right)\right\} \mathrm{d} s .
$$

By simple computation the expectation of $\left\langle\mathbf{M}^{G, g}\right\rangle_{t}$ vanishes as $\gamma \rightarrow 0$. We are now ready to define the family of the mean one exponential martingale $\left(\mathcal{Z}_{t}^{G, g}\right)_{t \geqslant 0}$ for all $G$ and $g$,

$$
\mathcal{Z}_{t}^{G, g}=\exp \left\{\gamma^{-d} \mathbf{M}_{t}^{G, g}-\frac{\gamma^{-2 d}}{2}\left\langle\mathbf{M}^{G, g}\right\rangle_{t}\right\}
$$

A summation by parts permits to rewrite the martingale $\gamma^{-d} \mathbf{M}_{t}^{G, g}$ as

$$
\begin{aligned}
\gamma^{-d} \mathbf{M}_{t}^{G, g}= & \gamma^{-d}\left\langle G_{t}, \pi_{t}^{\gamma}\right\rangle-\gamma^{-d}\left\langle G_{0}, \pi_{0}^{\gamma}\right\rangle-\gamma^{-d} \int_{0}^{t}\left\langle\partial_{s} G_{s}, \pi_{s}^{\gamma}\right\rangle \mathrm{d} s \\
& -\gamma^{-1} \int_{0}^{t}\left\{\sum_{i=1}^{d} \sum_{x \in \Lambda_{\gamma}}\left(\partial_{e_{i}}^{\gamma} G_{s}\right)(\gamma x)\left(\mathbf{J}_{x, x+e_{i}}+\mathcal{L}_{\gamma} \tau_{x} g_{i}^{\ell}\left(\eta_{s}, \alpha\right)\right)\right\} \mathrm{d} s \\
& +\gamma \sum_{i=1}^{d} \sum_{x \in \Lambda_{\gamma}}\left\{\left(\partial_{e_{i}}^{\gamma} G_{t}\right)(\gamma x) \tau_{x} g_{i}^{\ell}\left(\eta_{t}, \alpha\right)-\left(\partial_{e_{i}}^{\gamma} G_{0}\right)(\gamma x) \tau_{x} g_{i}^{\ell}\left(\eta_{0}, \alpha\right)\right\} \\
& -\gamma \int_{0}^{t}\left\{\sum_{i=1}^{d} \sum_{x \in \Lambda_{\gamma}} \partial_{s}\left(\partial_{e_{i}}^{\gamma} G_{s}\right)(\gamma x) \tau_{x} g_{i}^{\ell}\left(\eta_{s}, \alpha\right)\right\} \mathrm{d} s,
\end{aligned}
$$

where $\mathbf{J}_{x, x+e_{i}}$ is the current defined in (4.22). On the other hand, a summation by parts and Taylor expansion permit to rewrite the quadratic part $\frac{\gamma^{-2 d}}{2}\left\langle\mathbf{M}^{G, g}\right\rangle_{t}$ in the exponential martingale $\mathcal{Z}_{t}^{G, g}$ as

$$
\begin{aligned}
& \frac{\gamma^{-2 d}}{2}\left\langle\mathbf{M}^{G, g}\right\rangle_{t}=\mathrm{O}_{u}\left(\gamma^{-d+1}\right) \\
& \quad+\frac{1}{2} \sum_{k, j} \int_{0}^{t} \sum_{x \in \Lambda_{\gamma}}\left\{\left(\partial_{e_{k}} G_{s}(\gamma x)\right)\left(\partial_{e_{j}} G_{s}(\gamma x)\right) \sum_{i=1}^{d} C_{\gamma}\left(x, x+e_{i} ; \eta\right) \tau_{x} \mathcal{A}_{i, k}\left(\eta_{s}, g_{k}^{\ell}\right) \tau_{x} \mathcal{A}_{i, j}\left(\eta_{s}, g_{j}^{\ell}\right)\right\} \mathrm{d} s,
\end{aligned}
$$


where for $1 \leqslant i, k \leqslant d$,

$$
\mathcal{A}_{i, k}\left(\eta, g_{k}^{\ell}\right)=\left[-\nabla_{0, e_{i}} \eta(0) \delta_{i, k}+\nabla_{0, e_{i}} \Gamma g_{k}^{\ell}\right]
$$

and $\delta_{i, k}$ is the Kronecker delta. This time, however, it is not the density fields that appears in the exponential of $\mathcal{Z}_{t}^{G, g}$ but other local functions of the configurations $\eta$ and $\alpha$. The main step in proving Lemma 5.1 is to replace these local functions by functions of the density fields in order to recover the functionals $\widehat{F}_{a, c}^{G, m}$. Denote

$$
\begin{aligned}
F_{a, c}^{1, G}\left(\left(\pi_{t}^{\gamma}\right)_{t}\right)= & \left\langle G_{T}, \pi_{T}^{\gamma}\right\rangle-\left\langle G_{0}, \pi_{0}^{\gamma}\right\rangle-\int_{0}^{T}\left\langle\partial_{s} G_{s}, \pi_{s}^{\gamma}\right\rangle \mathrm{d} s+\sum_{k, m} \int_{0}^{T} \mathrm{~d} s \gamma^{d} \sum_{x \in \Lambda_{\gamma}}\left(\partial_{e_{k}} G_{s}\right)(\gamma x) \tau_{x} D_{k, m}\left(\pi_{s}^{\gamma} \star \iota_{a}(0)\right) \\
& \times \tau_{x}\left\{(2 c)^{-1}\left[\pi_{s}^{\gamma} \star \iota_{a}\left(c \gamma^{-1} e_{m}\right)-\pi_{s}^{\gamma} \star l_{a}\left(-c \gamma^{-1} e_{m}\right)\right]\right\} \\
& -\int_{0}^{T} \mathrm{~d} s \gamma^{d} \sum_{x \in \Lambda_{\gamma}}\left(\nabla^{\gamma} G_{s}(\gamma x) \cdot \frac{\beta}{2} \sigma\left(\pi_{s}^{\gamma} \star \iota_{a}(x)\right) \nabla^{\gamma}\left(J \star \pi_{s}^{\gamma}\left(\eta_{s}\right)(\gamma x)\right)\right), \\
F_{a}^{2, G}\left(\left(\pi_{t}^{\gamma}\right)_{t}\right)= & \int_{0}^{T} \mathrm{~d} s \gamma^{d} \sum_{x \in \Lambda_{\gamma}}\left(\nabla^{\gamma} G_{s}(\gamma x) \cdot \frac{1}{2} \sigma\left(\pi_{s}^{\gamma} \star \iota_{a}(x)\right) \nabla^{\gamma} G_{s}(\gamma x)\right) .
\end{aligned}
$$

Since $\eta_{s}^{\left[a \gamma^{-1}\right]}(x)=\left(\pi_{s}^{\gamma} \star \iota_{a}\right)(\gamma x)$, these two quantities are functions of the empirical density. Since $\gamma^{-d}\left\langle\mathbf{M}^{G, g}\right\rangle_{t}$ is bounded by some constant $M$, independent on $\gamma$ and $t$, using Hölder inequality, we have for $\varrho>1$

$$
\begin{aligned}
& \log \mathbf{E}^{Q_{\nu}^{\rho_{0}}}\left[\exp \left\{\gamma^{-d}\left(f_{0}^{m}+F_{a, c}^{1, G}-F_{a}^{2, G}\right)\right\}\right] \\
& =\log \mathbf{E}^{Q_{\gamma}^{\rho_{0}}}\left[\exp \left\{\gamma^{-d}\left(f_{0}^{\gamma, m}+F_{a, c}^{1, G}-F_{a}^{2, G}\right)\right\}\right]+\gamma^{-d}\left\{\int_{\Lambda} \log \left(\frac{1-m(r)}{1-\rho_{0}(r)}\right) \mathrm{d} r-\left\langle\log \left(\frac{1-m(\cdot)}{1-\rho_{0}(\cdot)}\right), L_{\gamma}\right\rangle\right\} \\
& \leqslant \frac{1}{\varrho} \log \mathbf{E}^{\mathbf{P}_{\nu_{\gamma}(\cdot)}}\left[\exp \left\{\varrho \gamma^{-d}\left(F_{a, c}^{1, G}\left(\left(\pi_{t}^{\gamma}(\eta)\right)_{t}\right)-F_{a}^{2, G}\left(\left(\pi_{t}^{\gamma}(\eta)\right)_{t}\right)\right)\right\}\left(\mathcal{Z}_{T}^{G, g}\right)^{-\varrho}\right] \\
& +\frac{\varrho-1}{\varrho} \log \mathbf{E}^{\mathbf{P}_{\gamma}^{m(.)}}\left[\mathcal{Z}_{T}^{\frac{\varrho}{Q-1} G, g}\right]+\frac{M}{2} \frac{\gamma^{-d}}{\varrho-1}+\gamma^{-d} \mathbf{o}(\gamma) \\
& =\frac{1}{\varrho} \log \mathbf{E}^{\mathbf{P}_{\gamma}^{m(\cdot)}}\left[\exp \left\{\varrho \gamma^{-d}\left(F_{a, c}^{1, G}\left(\left(\pi_{t}^{\gamma}(\eta)\right)_{t}\right)-F_{a}^{2, G}\left(\left(\pi_{t}^{\gamma}(\eta)\right)_{t}\right)\right)\right\}\left(\mathcal{Z}_{T}^{G, g}\right)^{-\varrho}\right]+\frac{M}{2} \frac{\gamma^{-d}}{\varrho-1}+\gamma^{-d} \mathrm{o}(\gamma),
\end{aligned}
$$

where $\mathcal{Z}_{t}^{\frac{\varrho}{\rho-1} G, g}$ is the mean one exponential martingale defined in (5.6) with $G \in \mathcal{C}^{1,2}([0, T] \times \Lambda)$. In the second inequality we used the fact that $\exp \left\{\gamma^{-d} f_{0}^{\gamma, m}(\cdot)\right\}$ is the density of $\mathrm{d} v_{\gamma}^{\rho_{0}}$ with respect to $\mathrm{d} v_{\gamma}^{m(.)}$ with

$$
f_{0}^{\gamma, m}\left(\mu_{0}\right)=\left\langle\log \left(\frac{m(\cdot)\left(1-\rho_{0}(\cdot)\right)}{\rho_{0}(\cdot)(1-m(\cdot))}\right), \mu_{0}\right\rangle+\left\langle\log \left(\frac{1-m(\cdot)}{1-\rho_{0}(\cdot)}\right), L_{\gamma}\right\rangle
$$

and $L_{\gamma}$ stands for the discrete approximation of the Lebesgue measure, $L_{\gamma}=\gamma^{d} \sum_{x \in \Lambda_{\gamma}} \delta_{\gamma x}$. We conclude the proof of Lemma 5.1, by applying Hölder inequality to the first term of the right-hand side of the last inequality, taking into account (5.10), (5.6) and showing the following lemma.

Lemma 5.4. There exist a positive constant $A_{1}$ and a sequence $\left(g_{N}\right)_{N>0}=\left(\left(g_{1, N}, \ldots, g_{d, N}\right)\right)_{N>0} \subset \mathbb{G}^{d}$ defined as in (5.4) such that for any continuous profile $m: \Lambda \rightarrow(0,1)$ and $G \in C^{1,2}([0, T] \times \Lambda)$

$$
\begin{aligned}
& \limsup _{N \rightarrow \infty} \underset{c \rightarrow 0}{\limsup \limsup } \limsup _{a \rightarrow 0} \gamma^{d} \log \mathbf{E}_{v_{\gamma}(.)}^{\mathbf{p}^{m(.)}}\left[\exp \left\{\varrho \gamma^{-d}\left(\mathbf{M}_{T}^{G, g_{N}}-F_{a, c}^{1, G}\right)\right\}\right] \leqslant A_{1}, \\
& \limsup _{N \rightarrow \infty} \limsup _{a \rightarrow 0} \limsup _{\gamma \rightarrow 0} \gamma^{d} \log \mathbf{E}^{\mathbf{P}_{v^{m(.)}}}\left[\exp \left\{\varrho \gamma^{-d}\left(\left(\frac{\gamma^{-d}}{2}\right)\left\langle\mathbf{M}^{G, g_{N}}\right\rangle_{T}-F_{a}^{2, G}\right)\right\}\right] \leqslant A_{1}
\end{aligned}
$$

for any $\varrho \in \mathbb{R}$. 
Proof. The (5.11) is similar to the proof of Theorem 4.5 and is therefore omitted (see also Lemmas 4.2 and 4.3 of [21]). We prove now (5.12). Let $\left(g_{N}\right)_{N \geqslant 1} \subset \mathbb{G}^{d}$ be the sequence, introduced in Section 4, satisfying (4.31). Consider the quadratic variation $\left\langle\mathbf{M}^{G, g_{N}}\right\rangle_{t}$ of the martingale $\mathbf{M}_{t}^{G, g_{N}}$ given by (5.8). From (4.15)

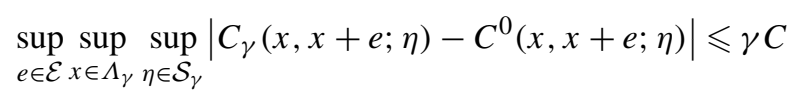

for some constant $C=C(\beta, J, \Phi, A)$. We may thus rewrite $\frac{\gamma^{-2 d}}{2}\left\langle\mathbf{M}^{G, g_{N}}\right\rangle$ as

$$
\frac{\gamma^{-2 d}}{2}\left\langle\mathbf{M}^{G, g_{N}}\right\rangle_{t}=\frac{1}{2} \sum_{x \in \Lambda_{\gamma}} \sum_{k, j} \int_{0}^{t}\left(\partial_{e_{k}} G_{s}(\gamma x)\right)\left(\partial_{e_{j}} G_{s}(\gamma x)\right) \tau_{x} \mathbb{W}_{j, k}^{g_{N}}\left(\eta_{s}\right) \mathrm{d} s+\mathrm{O}_{u}\left(\gamma^{-d+1}\right),
$$

where

$$
\mathbb{W}_{k, j}^{g}(\eta)=\sum_{i=1}^{d} C^{0}\left(0, e_{i} ; \eta\right) \mathcal{A}_{i, k}\left(\eta, g_{k}\right) \mathcal{A}_{i, j}\left(\eta, g_{j}\right), \quad 1 \leqslant k, j \leqslant d .
$$

The superexponential replacement lemma for the $\mathcal{L}_{\gamma}$ process, see Lemma 4.3, implies that $\mathbb{P}$ a.s

$$
\underset{a \rightarrow 0}{\limsup \limsup } \gamma_{\gamma \rightarrow 0} \gamma^{d} \log \mathbf{E}^{\mathbf{P}_{\gamma} v_{(.)}}\left\{\exp \left(\varrho \sum_{x \in \Lambda_{\gamma}} \int_{0}^{t} G_{s}(\gamma x)\left[\tau_{x} g\left(\eta_{s}, \alpha\right)-\tilde{g}\left(\eta_{s}^{\left[a \gamma^{-1}\right]}(x)\right)\right] \mathrm{d} s\right)\right\} \leqslant A_{3}^{\prime},
$$

where $\tilde{g}$ is defined in (4.16) and $A_{3}^{\prime}$ a suitable positive constant. Then by Schwartz inequality it is enough to prove that there exists a positive constant $A_{2}^{\prime}$ such that, $\mathbb{P}$ a.s. for $\varrho \in \mathbb{R}$

$$
\begin{aligned}
& \underset{N \rightarrow \infty}{\limsup } \limsup _{a \rightarrow 0} \limsup \gamma_{\gamma \rightarrow 0}^{d} \log \mathbf{E}^{\mathbf{P}_{\nu}^{m(.)}}\left\{\operatorname { e x p } \left(\varrho \sum_{x \in \Lambda_{\gamma}} \sum_{k, j} \int_{0}^{t}\left(\partial_{e_{k}} G_{s}(\gamma x)\right)\left(\partial_{e_{j}} G_{s}(\gamma x)\right)\right.\right. \\
& \left.\left.\quad \times\left[\widetilde{\mathbb{W}}_{k, j}^{g_{N}}\left(\eta_{s}^{\left[a \gamma^{-1}\right]}(x)\right)-\sigma_{k, j}\left(\eta_{s}^{\left[a \gamma^{-1}\right]}(x)\right)\right] \mathrm{d} s\right)\right\} \leqslant A_{2}^{\prime} .
\end{aligned}
$$

We now compute $\widetilde{\mathbb{W}}_{k, j}^{g_{N}}$. Using a change of variables, detailed balance condition (2.10) and properties (A.6), (A.7), we have for all density $0<\rho<1$

$$
\begin{aligned}
\frac{1}{2} \widetilde{\mathbb{W}}_{k, j}^{g_{N}}(\rho)= & \frac{1}{2} \mathbb{E}\left[\mathbf{E}^{\mu^{\alpha, \lambda_{0}(\rho)}}\left(C^{0}\left(0, e_{k} ; \eta\right)\left(\nabla_{0, e_{k}} \eta(0)\right)^{2}\right)\right] \delta_{k, j} \\
& +V_{\rho}\left(\mathbf{J}_{0, e_{k}}^{0}, \mathcal{L}^{0} g_{j, N}\right)+V_{\rho}\left(\mathbf{J}_{0, e_{j}}^{0}, \mathcal{L}^{0} g_{k, N}\right)+V_{\rho}\left(\mathcal{L}^{0} g_{j, N}, \mathcal{L}^{0} g_{k, N}\right) \\
= & V_{\rho}\left(\mathbf{J}_{0, e_{j}}^{0}+\mathcal{L}^{0} g_{j, N}, \mathbf{J}_{0, e_{k}}^{0}+\mathcal{L}^{0} g_{k, N}\right) .
\end{aligned}
$$

Therefore, in order to conclude the proof it is enough to show that for $1 \leqslant k, j \leqslant d$,

$$
\lim _{N \rightarrow \infty} \sup _{0 \leqslant \rho \leqslant 1}\left|V_{\rho}\left(\mathbf{J}_{0, e_{j}}^{0}+\mathcal{L}^{0} g_{j, N}, \mathbf{J}_{0, e_{k}}^{0}+\mathcal{L}^{0} g_{k, N}\right)-\frac{1}{2} \sigma_{k, j}(\rho)\right|=0 .
$$

Property (P) of the $\left(\psi_{e}\right)_{e \in \mathcal{E}}$ given in Appendix A and (A.8) permit to rewrite the quantity $-\frac{1}{2} \sigma_{k, j}(\rho)$ as

$$
-\frac{1}{2} \sigma_{k, j}(\rho)=V_{\rho}\left(\mathbf{J}_{0, e_{j}}^{0}+\mathcal{L}^{0} g_{j, N}, \sum_{\ell=1}^{d} D_{k, \ell}(\rho) \psi_{e_{\ell}}(\rho)\right),
$$

so that the expression in (5.18) is equal to

$$
V_{\rho}\left(\mathbf{J}_{0, e_{j}}^{0}+\mathcal{L}^{0} g_{j, N}, \mathbf{J}_{0, e_{k}}^{0}+\mathcal{L}^{0} g_{k, N}\right)-\frac{1}{2} \sigma_{k, j}(\rho)=V_{\rho}\left(\mathbf{J}_{0, e_{j}}^{0}+\mathcal{L}^{0} g_{j, N}, \mathbf{J}_{0, e_{k}}^{0}+\mathcal{L}^{0} g_{k, N}+\sum_{\ell=1}^{d} D_{k, \ell}(\rho) \psi_{e_{\ell}}(\rho)\right) .
$$


On the other hand, Schwartz inequality and Remark 7.20 in [8] allow us to introduce the terms $\sum_{m=1}^{d} D_{k, m}(\rho) \frac{\psi_{n, n}^{e_{m}}}{n}(\rho)$ in the right-hand side of the last quantity when $n \uparrow \infty$ uniformly in $0<\rho<1$. To complete the proof it remains to apply Schwartz inequality and to recall that $\left(g_{N}\right)$ satisfies (4.31). For more details, see the end of the proof of Lemma 4.8 where similar arguments are used.

\subsection{Exponential tightness and proof of Lemmas 5.2 and 5.3}

Standard arguments (cf. Section 10.4 of [14]) permit us to construct a sequence of compacts satisfying (5.3) from the following lemma.

Lemma 5.5. For each $\delta>0$ and smooth function $G: \Lambda \rightarrow \mathbb{R}, \mathbb{P}$ a.s.,

$$
\lim _{\varepsilon \rightarrow 0} \limsup _{\gamma \rightarrow 0} \gamma^{d} \log Q_{v_{\gamma}^{\rho_{0}}}\left\{\sup _{\substack{|s-t| \leqslant \varepsilon \\ 0 \leqslant s, t \leqslant T}}\left|\left\langle G, \pi_{t}\right\rangle-\left\langle G, \pi_{s}\right\rangle\right|>\delta\right\}=-\infty .
$$

For $\alpha \in \Omega_{D}$ and $0<t \leqslant T$ denote

$$
h_{t}^{\gamma, \alpha} \equiv h_{t}^{\gamma, \alpha}(G)=\gamma^{d-1} \int_{0}^{t} \mathrm{~d} s\left\{\sum_{i=1}^{d} \sum_{x \in \Lambda_{\gamma}}\left(\partial_{e_{i}}^{\gamma} G\right)(\gamma x) \mathbf{J}_{x, x+e_{i}}\left(\eta_{s}, \alpha\right)\right\} .
$$

The proof of Lemma 5.5 goes along the same lines of the proof of exponential tightness for non-gradient systems given in [14] and [30]. It relies on the following Lemma, of which we postpone the proof.

Lemma 5.6. For each $\delta>0$ and smooth function $G: \Lambda \rightarrow \mathbb{R}, \mathbb{P}$ a.s.,

$$
\lim _{\varepsilon \rightarrow 0} \limsup _{\gamma \rightarrow 0} \gamma^{d} \log \mathbf{P}_{\nu_{\gamma} \rho_{0}}\left\{\sup _{\substack{|s-t| \leqslant \varepsilon \\ 0 \leqslant s<t \leqslant T}}\left|h_{t}^{\gamma, \alpha}-h_{s}^{\gamma, \alpha}\right|>\delta\right\}=-\infty .
$$

Proof of Lemma 5.5. For $\gamma$ small enough, we have (see [14] page 271)

$$
\left\{\sup _{\substack{|s-t| \leqslant \varepsilon \\ 0 \leqslant s, t \leqslant T}}\left|\left\langle G, \pi_{t}\right\rangle-\left\langle G, \pi_{s}\right\rangle\right|>\delta\right\} \subset \bigcup_{k=0}^{\left[T \varepsilon^{-1}\right]}\left\{\sup _{k \varepsilon \leqslant t<(k+1) \varepsilon}\left|\left\langle G, \pi_{t}\right\rangle-\left\langle G, \pi_{k \varepsilon}\right\rangle\right|>\frac{\delta}{4}\right\},
$$

where for $a \in \mathbb{R},[a]$ stands for the integer part of $a$. Denote by $\left(S_{t}^{\gamma}\right)_{t \geqslant 0}$ the semigroup associated to the generator $\gamma^{-2} \mathcal{L}_{\gamma}$. For every $t \geqslant 0$ denote by $f_{t}^{\gamma}(\eta)$ the Radon-Nikodym derivative of the measure $v_{\gamma}^{\rho_{0}} S_{t}^{\gamma}$ with respect to $\nu_{\gamma}^{\rho_{0}}$. Using the fact that there is at most one particle per site, it is easy to prove that there exists some positive constant $C$ such that for all $\eta \in \mathcal{S}_{\gamma}$ and $t \geqslant 0, f_{t}^{\gamma}(\eta) \leqslant \exp \left(C \gamma^{-d}\right)$ and we have

$$
\begin{aligned}
Q_{v_{\gamma}} \rho_{0}\left\{\sup _{\substack{|s-t| \leqslant \varepsilon \\
0 \leqslant s, t \leqslant T}}\left|\left\langle G, \pi_{t}\right\rangle-\left\langle G, \pi_{s}\right\rangle\right|>\delta\right\} & \leqslant \sum_{k=0}^{\left[T \varepsilon^{-1}\right]} \mathbf{E}^{\mathbf{P}_{\gamma}^{\rho_{0}}}\left[f_{k \varepsilon}^{\gamma}(\eta) \mathbb{1}_{\left\{\sup _{0 \leqslant t<\varepsilon}\left|\left\langle G, \pi_{t}(\eta)\right\rangle-\left\langle G, \pi_{0}(\eta)\right\rangle\right|>\delta / 4\right\}}\right] \\
& \leqslant\left[T \varepsilon^{-1}\right] \exp \left(C \gamma^{-d}\right) \mathbf{P}_{\nu_{\gamma}^{\rho_{0}}}\left\{\sup _{0 \leqslant t<\varepsilon}\left|\left\langle G, \pi_{t}(\eta)\right\rangle-\left\langle G, \pi_{0}(\eta)\right\rangle\right|>\frac{\delta}{4}\right\} .
\end{aligned}
$$

Therefore, in order to prove the lemma, it is enough to show that

$$
\lim _{\varepsilon \rightarrow 0} \limsup _{\gamma \rightarrow 0} \gamma^{d} \log \mathbf{P}_{\nu_{\gamma}^{\rho_{0}}}\left\{\sup _{0 \leqslant t<\varepsilon}\left|\left\langle G, \pi_{t}(\eta)\right\rangle-\left\langle G, \pi_{0}(\eta)\right\rangle\right|>\frac{\delta}{4}\right\}=-\infty .
$$

On the other hand, from Lemma 5.6 and

$$
\limsup _{\gamma \rightarrow 0} \gamma^{d} \log \left(a_{\gamma}+b_{\gamma}\right) \leqslant \max \left\{\limsup _{\gamma \rightarrow 0} \gamma^{d} \log a_{\gamma} ; \limsup _{\gamma \rightarrow 0} \gamma^{d} \log b_{\gamma}\right\}
$$


it is easy to see that the limit (5.23) is equivalent to

$$
\lim _{\varepsilon \rightarrow 0} \limsup _{\gamma \rightarrow 0} \gamma^{d} \log \mathbf{P}_{\nu_{\gamma}}{ }_{\rho_{0}}\left\{\sup _{0 \leqslant t<\varepsilon}\left|\left\langle G, \pi_{t}(\eta)\right\rangle-\left\langle G, \pi_{0}(\eta)\right\rangle-h_{t}^{\gamma, \alpha}\right|>\delta\right\}=-\infty
$$

that for any $\delta>0$ and $\alpha \in \Omega_{D}$. Next observe that

$$
\left\langle G, \pi_{t}(\eta)\right\rangle-\left\langle G, \pi_{0}(\eta)\right\rangle-h_{t}^{\gamma, \alpha}=\mathbf{M}_{t}^{G, 0},
$$

where $\mathbf{M}_{t}^{G, 0}$ is the martingale in (5.7) with $g=0$. Then by exponential Chebyshev inequality, we have

$$
\begin{aligned}
& \limsup _{\varepsilon \rightarrow 0} \limsup _{\gamma \rightarrow 0} \gamma^{d} \log \mathbf{P}_{\nu_{\gamma}^{\rho_{0}}}\left\{\sup _{0 \leqslant t<\varepsilon}\left|\left\langle G, \pi_{t}(\eta)\right\rangle-\left\langle G, \pi_{0}(\eta)\right\rangle-h_{t}^{\gamma, \alpha}\right|>\delta\right\} \\
& \leqslant-a \delta+\limsup _{\varepsilon \rightarrow 0} \limsup _{\gamma \rightarrow 0} \gamma^{d} \log \mathbf{E}^{\mathbf{P}_{\gamma} \rho_{\gamma}}\left\{\sup _{0 \leqslant t<\varepsilon} \exp \left(\gamma^{-d}\left|\mathbf{M}_{t}^{a G, 0}\right|\right)\right\}
\end{aligned}
$$

for all $a>0$. Since $\mathrm{e}^{|x|} \leqslant \mathrm{e}^{x}+\mathrm{e}^{-x}$ and $\mathbf{M}_{t}^{-G, 0}=-\mathbf{M}_{t}^{G, 0}$ then from (5.24), it is enough to prove

$$
\limsup _{\varepsilon \rightarrow 0} \limsup _{\gamma \rightarrow 0} \gamma^{d} \log \mathbf{E}^{\mathbf{P}_{\gamma}^{\rho_{0}}}\left\{\sup _{0 \leqslant t<\varepsilon} \exp \left(\gamma^{-d} \mathbf{M}_{t}^{a G, 0}\right)\right\} \leqslant C_{0}
$$

for some $C_{0} \in \mathbb{R}$ independent of $a$. We now express the exponential of the martingale $\mathbf{M}_{t}^{a G, 0}$ through the quadratic variation of $\mathbf{M}_{t}^{a G, 0}$ and the exponential martingale $\mathcal{Z}_{t}^{a G, 0}$ defined in (5.6) with $g=0$. For $a>0$ and $0 \leqslant t<\varepsilon$, we have

$$
\begin{aligned}
\exp \left(\gamma^{-d} \mathbf{M}_{t}^{a G, 0}\right) & =\mathcal{Z}_{t}^{a G, 0} \times \exp \left(\frac{\gamma^{-2 d}}{2}\left\langle\mathbf{M}^{a G, 0}\right\rangle_{t}\right) \\
& \leqslant \exp \left(C_{1} a^{2} \gamma^{-d} \varepsilon\right) \mathcal{Z}_{t}^{a G, 0}
\end{aligned}
$$

with some constant $C_{1}=C_{1}(G)$ such that $\gamma^{-d}\left\langle\mathbf{M}^{a G, 0}\right\rangle_{t}$ is bounded by $a^{2} C_{1} t$. Finally, we just have to apply the maximal martingale inequality and (5.27) to get

$$
\lim _{\varepsilon \rightarrow 0} \limsup _{\gamma \rightarrow 0} \gamma^{d} \log \mathbf{E}^{\mathbf{P}_{\gamma}^{\rho_{0}}}\left\{\sup _{0 \leqslant t<\varepsilon} \exp \left(\gamma^{-d} \mathbf{M}_{t}^{a G, 0}\right)\right\}=0 .
$$

This concludes the proof of the lemma.

Proof of Lemma 5.6. We follow some arguments used in Section 7.6 of [14]. For $\varepsilon>0$ small enough let $A_{\varepsilon}=$ $\frac{1}{32 \sqrt{\varepsilon} \log \left(\varepsilon^{-1}\right)}$, by exponential Chebyshev inequality

$$
\gamma^{d} \log \mathbf{P}_{\nu_{\gamma}}\left\{\sup _{\substack{|s-t| \leqslant \varepsilon \\ 0 \leqslant s<t \leqslant T}}\left|h_{t}^{\gamma, \alpha}-h_{s}^{\gamma, \alpha}\right|>\delta\right\} \leqslant-\delta A_{\varepsilon}+\gamma^{d} \log \mathbf{E} \mathbf{P}_{\gamma}^{\rho_{0}}\left\{\exp \left(\gamma^{-d} A_{\varepsilon} \sup _{\substack{|s-t| \leqslant \varepsilon \\ 0 \leqslant s<t \leqslant T}}\left|h_{t}^{\gamma, \alpha}-h_{s}^{\gamma, \alpha}\right|\right)\right\} .
$$

Therefore to conclude the proof of the lemma, it is enough to show that

$$
\limsup _{\varepsilon \rightarrow 0} \gamma^{d} \log \mathbf{E} \mathbf{E}_{\gamma}^{\nu_{\gamma}}\left\{\exp \left(\gamma^{-d} A_{\varepsilon} \sup _{\substack{|s-t| \leqslant \varepsilon \\ 0 \leqslant s<t \leqslant T}}\left|h_{t}^{\gamma, \alpha}-h_{s}^{\gamma, \alpha}\right|\right)\right\} \leqslant C
$$

for some constant $C=C(G, T, \beta)$. The proof of this lemma is essentially the same as the one of Corollary 7.6.3 in [14]. The proof of [14] uses the Garcia-Rodemich-Rumsey inequality and it relies on the following lemma.

Lemma 5.7. There exist two positive constants $C_{1}$ and $C_{2}$ such that for each $0 \leqslant s<t \leqslant T$

$$
\mathbf{E}^{\mathbf{P}_{\nu}^{\rho_{\gamma}}}\left\{\exp \left(\gamma^{-d}|t-s|^{-1 / 2}\left|h_{t}^{\gamma, \alpha}-h_{s}^{\gamma, \alpha}\right|\right)\right\} \leqslant 2 \exp \left(C_{1} \sum_{i=1}^{d} \sum_{x \in \Lambda_{\gamma}}\left[\left(\partial_{e_{i}}^{\gamma} G\right)(\gamma x)\right]^{2}+C_{2}(|t-s|+1) \gamma^{-d}\right) .
$$


Proof. Denote

$$
V_{\gamma}^{\alpha}(G, \eta) \equiv \sum_{i=1}^{d} \sum_{x \in \Lambda_{\gamma}}\left(\partial_{e_{i}}^{\gamma} G\right)(\gamma x) \mathbf{J}_{x, x+e_{i}}(\eta, \alpha) .
$$

Fix a constant profile $0<\rho<1$, for $s \in[0, T]$ denote by $f_{s}^{\gamma}$ the Radon-Nikodym derivative of the measure $v_{\gamma}^{\rho_{0}} S_{s}^{\gamma}$ with respect to the Gibbs measure $\mu_{\gamma}^{\alpha, \lambda_{0}(\rho)}$, where $\left(S_{t}^{\gamma}\right)$ is the semigroup associated to the generator $\gamma^{-2} \mathcal{L}_{\gamma}$. Then the left-hand side of (5.29) can be rewritten as

$$
\mathbf{E}^{\mathbf{P}_{\gamma}^{\alpha, \lambda_{0}(\rho)}}\left[f_{s}^{\gamma}\left(\eta_{0}\right) \times \exp \left(\gamma^{-1}|t-s|^{-1 / 2}\left|\int_{0}^{t-s} V_{\gamma}^{\alpha}\left(G, \eta_{u}\right) \mathrm{d} u\right|\right)\right] .
$$

Using the fact that there is at most one particle per site, it is easy to prove that there exists some positive constant $C$ such that for all $\eta \in \mathcal{S}_{\gamma}, f_{s}^{\gamma}(\eta) \leqslant \exp \left(C \gamma^{-d}\right)$. Since $\mathrm{e}^{|x|} \leqslant \mathrm{e}^{x}+\mathrm{e}^{-x}$ it is enough to estimate

$$
\exp \left(C \gamma^{-d}\right) \mathbf{E}^{\mathbf{P}_{\mu}^{\alpha, \lambda_{0}(\rho)}}\left[\exp \left(\gamma^{-1}|t-s|^{-1 / 2} \int_{0}^{t-s} V_{\gamma}^{\alpha}\left(G, \eta_{u}\right) \mathrm{d} u\right)\right] .
$$

By Lemma 3.6. of [21] (with $M=\frac{1}{2}$ ), we have that (5.31) is bounded by

$$
\exp \left(C \gamma^{-d}\right) \exp \left(|t-s|\left(\lambda_{\gamma}^{s, t}(G)+2 C_{0}^{\prime} \gamma^{-d}\right)\right)
$$

with positive constant $C_{0}^{\prime}$ given in Lemma 3.6 of [21] and $\lambda_{\gamma}^{s, t}(G)$ is given by the variational formula

$$
\lambda_{\gamma}^{s, t}(G)=\sup \left\{\gamma^{-1}|t-s|^{-1 / 2} \int V_{\gamma}^{\alpha}(G, \eta) f(\eta) \mu_{\gamma}^{\alpha, \lambda_{0}(\rho)}(\mathrm{d} \eta)+\frac{1}{2} \gamma^{-2}\left\langle\mathcal{L}_{\gamma}^{0} \sqrt{f}, \sqrt{f}\right\rangle_{\mu_{\gamma}^{\alpha, \lambda_{0}(\rho)}}\right\},
$$

where the supremum is carried over all probability densities $f$ with respect to $\mu_{\gamma}^{\alpha, \lambda_{0}(\rho)}$. We now split the current as

$$
\mathbf{J}_{x, x+e}=\mathbf{J}_{x, x+e}^{0}+\left[\mathbf{J}_{x, x+e}-\mathbf{J}_{x, x+e}^{0}\right],
$$

where $\mathbf{J}_{x, x+e}^{0}$ is defined in (4.10). From (4.15), see also Lemma 3.4 in [21], one easily obtains that

$$
\mathbf{J}_{x, x+e}-\mathbf{J}_{x, x+e}^{0}=-\beta \gamma \Phi^{\prime}\left(\left(\nabla_{x, x+e} H_{0}^{\alpha}\right)(\eta)\right)(\eta(x)-\eta(x+e))^{2}\left(\left(\left(\partial_{e}^{\gamma} J\right) \star \eta\right)(x)\right)+\mathrm{O}_{u}\left(\gamma^{2}\right),
$$

where the function $\Phi$ is defined in (2.10). Then inserting (5.34) in (5.30), we obtain from (5.32)

$$
\begin{aligned}
\lambda_{\gamma}^{s, t}(G) \leqslant & \sup \left\{|t-s|^{-1 / 2} \sum_{i=1}^{d} \sum_{x \in \Lambda_{\gamma}} \int\left[\gamma^{-1}\left(\partial_{e_{i}}^{\gamma} G\right)(\gamma x) \mathbf{J}_{x, x+e_{i}}^{0}(\eta, \alpha)+\left|\left(\partial_{e_{i}}^{\gamma} G\right)(\gamma x)\right| C_{2}^{\prime}\right] f(\eta) \mu_{\gamma}^{\alpha, \lambda_{0}(\rho)}(\mathrm{d} \eta)\right. \\
& \left.+\frac{1}{2} \gamma^{-2}\left\langle\mathcal{L}_{\gamma}^{0} \sqrt{f}, \sqrt{f}\right\rangle_{\mu_{\gamma}^{\alpha, \lambda_{0}(\rho)}}\right\},
\end{aligned}
$$

where $C_{2}^{\prime}$ is a constant. Next we use the integration by parts formula for the current $\mathbf{J}_{x, x+e}^{0}$, for each $x \in \Lambda_{\gamma}$

$$
\int \mathbf{J}_{x, x+e}^{0}(\eta) f(\eta) \mathrm{d} \mu_{\gamma}^{\alpha, \lambda_{0}(\rho)}(\eta)=\int C^{0}(x, x+e ; \eta) \eta(x+e)\left[\left(\nabla_{x, x+e} f\right)(\eta)\right] \mathrm{d} \mu_{\gamma}^{\alpha, \lambda_{0}(\rho)}(\eta) .
$$

By the elementary inequality $2 u v \leqslant A u^{2}+A^{-1} v^{2}$, we obtain for fixed $x \in \Lambda_{\gamma}$

$$
\begin{aligned}
\mid t & -\left.s\right|^{-1 / 2}\left\{\gamma^{-1}\left(\partial_{e}^{\gamma} G\right)(\gamma x) \int \mathbf{J}_{x, x+e}^{0}(\eta, \alpha) f(\eta) \mu_{\gamma}^{\alpha, \lambda_{0}(\rho)}(\mathrm{d} \eta)+\left|\left(\partial_{e}^{\gamma} G\right)(\gamma x)\right| C_{2}^{\prime}\right\} \\
& \leqslant \gamma^{-2} \frac{A}{2} \int C^{0}(x, x+e ; \eta)\left[\sqrt{f\left(\eta^{x, x+e}\right)}-\sqrt{f(\eta)}\right]^{2} \mu_{\gamma}^{\alpha, \lambda_{0}(\rho)}(\mathrm{d} \eta)+\frac{A}{2}\left(C_{2}^{\prime}\right)^{2}+C_{3} \frac{\left[\left(\partial_{e}^{\gamma} G\right)(\gamma x)\right]^{2}}{A|t-s|}
\end{aligned}
$$

for all $A>0$, for some finite constant $C_{3}$. To conclude the proof of the lemma it remains to take the sum over $x \in \Lambda_{\gamma}$, $e \in \mathcal{E}$ and to choose $A$ small enough. 
Proof of Lemma 5.2. Let $\mu \in D\left([0, T], \mathcal{M}_{1}(\Lambda)\right)$ such that $\mathcal{I}_{0}(\mu(\cdot, \cdot))<+\infty$, then for all $t \in[0, T], \mu(t, \cdot)$ is absolutely continuous with respect to the Lebesgue measure, denote by $\mu(t, r)=\rho(t, r) \mathrm{d} r$. We have that $\left(\mu \star l_{a}\right)(\cdot, \cdot)$ converges to $\mu(\cdot, \cdot)$ in $L^{1}([0, T] \times \Lambda)$. This implies that there exists a subsequence $\left(a_{k}\right)_{k \in \mathbb{N}}$ such that $\left(\mu \star l_{a_{k}}\right)(\cdot, \cdot)$ converges when $k \rightarrow \infty$ to $\mu(\cdot, \cdot)$ a.e. for the Lebesgue measure in $[0, T] \times \Lambda$. We first note that,

$$
\mathcal{I}_{\text {init }}(\rho(0, \cdot))=\sup _{\substack{m \in C^{0}(\Lambda) \\ 0<m<1}} f_{0}^{m}(\rho(0, \cdot)),
$$

where

$$
f_{0}^{m}(\rho(0, \cdot))=\int_{\Lambda}\left(\frac{m(r)\left(1-\rho_{0}(r)\right)}{\rho_{0}(r)(1-m(r))}\right) \rho(0, r) \mathrm{d} r+\int_{\Lambda} \log \left(\frac{1-m(r)}{1-\rho_{0}(r)}\right) \mathrm{d} r .
$$

Let $M$ be a positive constant and suppose that $\mathcal{I}_{\text {dyn }}(\mu(\cdot, \cdot)) \geqslant M$. Let $\varepsilon>0$, then from (3.2), there exists $G \in$ $C^{1,2}([0, T] \times \Lambda)$ such that

$$
M-\varepsilon \leqslant \mathcal{J}_{G}(\rho(\cdot, \cdot)) .
$$

To prove the lemma, we only need to show that, there exist $k_{0} \in \mathbb{N}$ and $0<c_{0}<1$, such that for all $k \geqslant k_{0}$ and $0<c \leqslant c_{0}$,

$$
M-2 \varepsilon \leqslant \widehat{F}_{a_{k}, c}^{G, m}(\mu(\cdot, \cdot))-f_{0}^{m}\left(\mu_{0}\right)+R\left(c, a_{k}, G\right),
$$

where $R\left(c, a_{k}, G\right)$ is such that $\lim _{c \rightarrow 0} \lim _{k \rightarrow \infty} R\left(c, a_{k}, G\right)=0$. The proof of (5.36) follows from the continuity of $D(\cdot)$ and bounded convergence theorem (for details, see [25] pages 735 and 736 where the same inequality is proved).

Proof of Lemma 5.3. Since (2.23) we have for $\mu(t, \mathrm{~d} r)=\rho(t, r) \mathrm{d} r$

$$
\mathcal{I}_{0}(\mu(\cdot, \cdot)) \leqslant \frac{1}{C} \sup _{G}\left\{2 C \int_{0}^{T} \int_{\Lambda} D(\rho) \nabla \rho \nabla G-\iint_{0}^{T} \rho(1-\rho)|\nabla G|^{2}\right\} .
$$

It is therefore enough to prove the lemma with the functional

$$
\mathcal{I}_{0}^{C}(\mu(\cdot, \cdot))=\sup _{G}\left\{2 C \int_{0}^{T} \int_{\Lambda} D(\rho) \nabla \rho \nabla G-\iint_{0}^{T} \rho(1-\rho)|\nabla G|^{2}\right\},
$$

instead of $\mathcal{I}_{0}$. Denote by $R$ the function $R(\rho)=\rho(1-\rho)$. For $a>0$, denote by $\iota_{a}$ the approximation of the identity defined by (5.1). For $G(\cdot, \cdot) \in C^{1,2}([0, T] \times \Lambda)$ and $0<a, c<1$ consider the family of functionals given by

$$
\begin{aligned}
\bar{F}_{a, c}^{G}(\mu(\cdot, \cdot))= & 2 C \sum_{1 \leqslant k, j \leqslant d} \int_{0}^{T} \mathrm{~d} s \int_{\Lambda} \mathrm{d} r\left(\partial_{e_{k}} G_{s}\right)(r) D_{k, j}\left(\left(\mu_{s} \star \iota_{a}\right)(r)\right) \\
& \times(2 c)^{-1}\left[\left(\mu_{s} \star \iota_{a}\right)\left(r+c e_{j}\right)-\left(\mu_{s} \star \iota_{a}\right)\left(r-c e_{j}\right)\right] \\
& -\sum_{1 \leqslant k \leqslant d} \int_{0}^{T} \mathrm{~d} s \int_{\Lambda} \mathrm{d} r\left(\partial_{e_{k}} G_{s}\right)^{2}(r) R\left(\left(\mu_{s} \star \iota_{a}\right)(r)\right),
\end{aligned}
$$

so that for each $\mu(\cdot, \cdot)$,

$$
\lim _{c \rightarrow 0} \limsup _{a \rightarrow 0} \sup _{G} \bar{F}_{a, c}^{G}(\mu(\cdot, \cdot))=\mathcal{I}_{0}^{C}(\mu(\cdot, \cdot))
$$

in the sense that if the right-hand side is infinite then the left-hand side is infinite as well. Let 


$$
\begin{aligned}
\bar{F}_{a, c, \gamma}^{G}(\mu(\cdot, \cdot))= & 2 C \sum_{k, m} \int_{0}^{T} \mathrm{~d} s \gamma^{d} \sum_{x \in \Lambda_{\gamma}}\left(\partial_{e_{k}} G_{s}\right)(\gamma x) \tau_{x} D_{k, m}\left(\mu_{s} \star \iota_{a}(0)\right) \\
& \times \tau_{x}\left\{(2 c)^{-1}\left[\mu_{s} \star \iota_{a}\left(c \gamma^{-1} e_{m}\right)-\mu_{s} \star \iota_{a}\left(-c \gamma^{-1} e_{m}\right)\right]\right\} \\
& -\sum_{k=1}^{d} \int_{0}^{T} \mathrm{~d} s \gamma^{d} \sum_{x \in \Lambda_{\gamma}}\left(\partial_{e_{k}} G_{s}(\gamma x)\right)^{2} R\left(\mu_{s} \star \iota_{a}(x)\right) .
\end{aligned}
$$

To prove the lemma is therefore enough to show that there exists some positive constant $A_{0}$ such that, for any $G$

$$
\lim _{c \rightarrow 0} \limsup _{a \rightarrow 0} \limsup _{\gamma \rightarrow 0} \gamma^{d} \log \mathbf{E}^{\mathbf{P}_{\nu} \rho_{0}}\left[\exp \left(\gamma^{-d} \bar{F}_{a, c, \gamma}^{G}\right)\right] \leqslant A_{0}
$$

This last limit can be proved by using the same arguments to obtain the energy estimate (cf. [14,26,29]).

\section{Lower bound}

In this section we establish the large deviations lower bound.

Definition of $\mathcal{D}^{0}$. Denote by $\mathcal{D}^{0}$ the class of trajectories $\mu \in D\left([0, T], \mathcal{M}_{1}^{0}(\Lambda)\right)$ such that for $t \in[0, T], \mu(t, \mathrm{~d} r)=$ $\rho(t, r) \mathrm{d} r$ and there exists $V \in C^{1,2}([0, T] \times \Lambda)$ and a function $m: \Lambda \rightarrow(0,1)$ so that the profile given by the density $\rho(\cdot, \cdot)$ is the weak solution of Eq. (4.6) with initial condition $\rho(0, \cdot)=m(\cdot)$.

The strategy of the proof of the lower bound consists of two steps. We first prove that for each $\mu \in \mathcal{D}^{0}$ and each neighborhood $\mathcal{N}_{\mu}$ of $\mu$ in $D\left([0, T], \mathcal{M}_{1}(\Lambda)\right)$, for almost all disorder $\alpha \in \Omega_{D}$

$$
\liminf _{\gamma \rightarrow 0} \gamma^{d} \log Q_{\gamma}\left\{\mathcal{N}_{\mu}\right\} \geqslant-\mathcal{I}(\mu(\cdot, \cdot)) .
$$

The proof of the lower bound is then accomplished by showing, see Section 7, that for any $\mu(\cdot, \cdot) \in D\left([0, T], \mathcal{M}_{1}(\Lambda)\right)$ with $\mathcal{I}(\mu)<\infty$ we can find a sequence of $\mu^{k} \in \mathcal{D}^{0}$ such that $\lim _{k \rightarrow \infty} \mu^{k}=\mu$ in $D\left([0, T], \mathcal{M}_{1}(\Lambda)\right)$ and $\lim _{k \rightarrow \infty} \mathcal{I}\left(\mu^{k}(\cdot, \cdot)\right)=\mathcal{I}(\mu(\cdot, \cdot))$. The lower bound (6.1) depends on establishing laws of large numbers, in hydrodynamic scaling, for weak random perturbations of the original process, the one having generator (2.7), and controlling by the Girsanov formula the relative entropies of the processes that go with these perturbations. Let $V \in C^{1,2}([0, T] \times \Lambda)$ and $v=\left(v_{1}, \ldots, v_{d}\right) \in \mathbb{G}^{d}$ be a vector of local random function defined at the beginning of Section 4. Let $\mathbf{P}_{v_{\gamma}, v}^{V, v}$ be the probability measure on the path space $D\left([0, T], \mathcal{S}_{\gamma}\right)$ corresponding to the Markov process $\left(\eta_{t}\right)_{t \geqslant 0}$ with generator $\gamma^{-2} \mathcal{L}_{\gamma}^{V, v}$, see (4.4), starting from the Bernoulli product measure $v_{\gamma}^{m}, 0<m(\gamma x)<1$, for $x \in \Lambda_{\gamma}$. Recall from Section 2 that we denoted by $\mathbf{P}_{\nu_{\gamma}}$ the law of $\gamma^{-2} \mathcal{L}_{\gamma}$ process with initial condition $v_{\gamma}^{\rho_{0}(\cdot)}$, being $\rho_{0}: \Lambda \rightarrow[0,1]$ the initial fixed profile. Let $H\left(\mathbf{P}_{v_{\gamma}^{m}}^{V, v} \mid \mathbf{P}_{v_{\gamma}}\right)$ be the entropy of the law $\mathbf{P}_{v_{\gamma}^{m}}^{V, v}$ of the perturbed process with respect to $\mathbf{P}_{v_{\gamma}^{\rho_{0}}}$. We will prove in Lemma 6.1 that $\gamma^{d} H\left(\mathbf{P}_{v_{\gamma}^{m}}^{V, v} \mid \mathbf{P}_{v_{\gamma}}\right)$ as $\gamma \rightarrow 0$ converges $\mathbb{P}$ almost surely to the sum of the initial entropy and the dynamical contribution $\mathcal{I}^{v}$ depending on the local function $v \in \mathbb{G}^{d}$ and $V$. Then in Lemma 6.3, we will show that the lower bound defined as the infimum over $v$ of $\mathcal{I}^{v}$ coincides with the upper bound rate for $\mu \in \mathcal{D}^{0}$. Since, see (4.21),

$$
\frac{\mathrm{d} \mathbf{P}_{v_{\gamma}^{m}}^{V, v}}{\mathrm{~d} \mathbf{P}_{\nu_{\gamma}}^{\rho_{0}}}=\frac{\mathrm{d} v_{\gamma}^{m}}{\mathrm{~d} \nu_{\gamma}^{\rho_{0}}}\left(\eta_{0}\right) \times Z_{T}^{\frac{V}{2}, \frac{v}{2}} \exp \left\{\gamma^{-d} O_{V, v}(\gamma)\right\}
$$

we have

$$
H\left(\mathbf{P}_{v_{\gamma}^{m}}^{V, v} \mid \mathbf{P}_{\nu_{\gamma}^{\rho_{0}}}\right)=\int \log \left(\frac{\mathrm{d} v_{\gamma}^{m}}{\mathrm{~d} \nu_{\gamma}^{\rho_{0}}}(\eta)\right) \mathrm{d} v_{\gamma}^{m}(\eta)+\mathbf{E}_{\nu_{\gamma}^{m}}^{\mathbf{P}^{V, v}}\left[\log \left(Z_{T}^{\frac{V}{2}, \frac{v}{2}}\right)\right]+\gamma^{-d} \mathrm{O}_{V, v}(\gamma) .
$$

For $k, j \in\{1, \ldots, d\}$, consider the local function $\mathbb{W}_{k, j}^{v}(\eta)$ defined replacing $g$ with $v$ in (5.15), 


$$
\begin{aligned}
\mathbb{W}_{k, j}^{v}(\eta) & =\sum_{i=1}^{d} C^{0}\left(0, e_{i} ; \eta\right) \mathcal{A}_{i, k}\left(\eta, v_{k}\right) \mathcal{A}_{i, j}\left(\eta, v_{j}\right) \\
& =\sum_{i=1}^{d} C^{0}\left(0, e_{i} ; \eta\right)\left[-\nabla_{0, e_{i}} \eta(0) \delta_{i, k}+\nabla_{0, e_{i}} \Gamma v_{k}\right] \times\left[-\nabla_{0, e_{i}} \eta(0) \delta_{i, j}+\nabla_{0, e_{i}} \Gamma v_{j}\right]
\end{aligned}
$$

For $\rho \in[0,1]$, see (4.16), denote

$$
\widetilde{\mathbb{W}}_{k, j}^{v}(\rho)=\mathbb{E}\left[\mathbf{E}^{\mu^{\alpha, \lambda_{0}(\rho)}}\left(\mathbb{W}_{k, j}^{v}\right)\right]
$$

and by $\widetilde{\mathbb{W}}^{v}(\rho)$ the associate matrix. Define the (non-random) rate functional

$$
\mathcal{I}^{v}(\mu)=\frac{1}{8} \int_{0}^{T} \mathrm{~d} t \int_{\Lambda}\left((\nabla V)(t, r) \cdot \widetilde{\mathbb{W}}^{v}(\rho(t, r))(\nabla V)(t, r)\right) \mathrm{d} r, \quad k, j \in\{1, \ldots, d\}
$$

where $\mu(t, \mathrm{~d} r)=\rho(t, r) \mathrm{d} r, t \in[0, T]$. Moreover, for each continuous $m: \Lambda \rightarrow(0,1)$, denote by $\rho^{m, V}(\cdot, \cdot)$ the weak solution of (4.6) with initial condition $\rho^{m, V}(0, \cdot)=m(\cdot)$. The $\mu^{m, V}(\cdot, \cdot)$ stands for the path on $\mathcal{D}^{0}$ having density $\rho^{m, V}(\cdot, \cdot)$. We have:

Lemma 6.1. For any $V(\cdot, \cdot) \in C^{1,2}([0, T] \times \Lambda)$ and $v \in \mathbb{G}^{d}$, for any continuous function $m: \Lambda \rightarrow(0,1)$, we have $\mathbb{P}$ a.s.

$$
\lim _{\gamma \rightarrow 0} \gamma^{d} H\left(\mathbf{P}_{v_{\gamma}^{m}}^{V, v} \mid \mathbf{P}_{\nu_{\gamma}}\right)=\mathcal{I}_{\text {init }}\left(\mu^{m, V}(0, \cdot)\right)+\mathcal{I}^{\frac{v}{2}}\left(\mu^{m, V}(\cdot, \cdot)\right)
$$

Proof. The strategy to show (6.5) is the same as in Lemma 7.5.4. of [14]. The contribution to (6.5) at time $t=0$ is easy to compute since $\nu_{\gamma}^{m(\cdot)}$ is a Bernoulli product measure with $m(\gamma x)$ as the probability of the site $x$ being occupied. We obtain

$$
\begin{aligned}
\lim _{\gamma \rightarrow 0} \gamma^{d} \int \log \left(\frac{\mathrm{d} \nu_{\gamma}^{m}}{\mathrm{~d} \nu_{\gamma}^{\rho_{0}}}(\eta)\right) \mathrm{d} v_{\gamma}^{m}(\eta) & =\int \log \left(\frac{m(r)}{\rho_{0}(r)}\right) m(r) \mathrm{d} r+\int \log \left(\frac{1-m(r)}{1-\rho_{0}(r)}\right)(1-m(r)) \mathrm{d} r \\
& =\mathcal{I}_{\text {init }}\left(\mu^{m, V}(0, \cdot)\right) .
\end{aligned}
$$

Applying similar arguments as in Section 4 one obtains

$$
\lim _{\gamma \rightarrow 0} \gamma^{d} \mathbf{E}_{v_{\gamma}^{m}}^{\mathbf{P}^{V, v}}\left[\log \left(Z_{T}^{\frac{V}{2}, \frac{v}{2}}\right)\right]=\mathcal{I}^{\frac{v}{2}}\left(\mu^{m, V}(\cdot, \cdot)\right) .
$$

For $\mu \in \mathcal{D}^{0}$ with associate profile $\rho(\cdot, \cdot)$, denote

$$
\mathcal{I}_{\text {lower }}(\mu(\cdot, \cdot))=\frac{1}{8} \inf _{v \in \mathbb{G}^{d}} \int_{0}^{T} \mathrm{~d} t \int_{\Lambda}\left((\nabla V)(t, r) \cdot \widetilde{\mathbb{W}}^{v}(\rho(t, r))(\nabla V)(t, r)\right) \mathrm{d} r .
$$

Lemma 6.2. For each $\mu \in \mathcal{D}^{0}$ with density profile $\rho(\cdot, \cdot)$, we have

$$
\mathcal{I}_{\text {lower }}(\mu(\cdot, \cdot))=\frac{1}{8} \int_{0}^{T} \mathrm{~d} t \int_{\Lambda}((\nabla V)(t, r) \cdot \sigma(\rho(t, r))(\nabla V)(t, r)) \mathrm{d} r .
$$

Proof. Since $\frac{1}{2} \sigma_{k, j}(\rho)=\chi(\rho) D_{k, j}(\rho)$, it is enough to show that

$$
\sup _{\rho \in[0,1]}\left|\frac{1}{2} \inf _{v \in \mathbb{G}^{d}} \widetilde{\mathbb{W}}_{k, j}^{v}(\rho)-\chi(\rho) D_{k, j}(\rho)\right|=0 .
$$

We have from (5.17) and (5.19) 


$$
\begin{aligned}
\frac{1}{2} \widetilde{\mathbb{W}}_{k, j}^{v}(\rho)-\chi(\rho) D_{k, j}(\rho)= & V_{\rho}\left(\mathbf{J}_{0, e_{j}}^{0}+\mathcal{L}^{0} v_{j}, \mathbf{J}_{0, e_{k}}^{0}+\mathcal{L}^{0} v_{k}+\sum_{\ell=1}^{d} D_{k, \ell}(\rho) \psi_{e_{\ell}}\right) \\
= & V_{\rho}\left(\mathbf{J}_{0, e_{j}}^{0}+\mathcal{L}^{0} v_{j}+\sum_{\ell=1}^{d} D_{j, \ell}(\rho) \psi_{e_{\ell}}, \mathbf{J}_{0, e_{k}}^{0}+\mathcal{L}^{0} v_{k}+\sum_{\ell=1}^{d} D_{k, \ell}(\rho) \psi_{e_{\ell}}\right) \\
& -V_{\rho}\left(\sum_{\ell=1}^{d} D_{j, \ell}(\rho) \psi_{e_{\ell}}, \mathbf{J}_{0, e_{k}}^{0}+\mathcal{L}^{0} v_{k}+\sum_{\ell=1}^{d} D_{k, \ell}(\rho) \psi_{e_{\ell}}\right) .
\end{aligned}
$$

From Theorem 7.22 of [8], we can find a sequence $\left(v_{N}\right) \in \mathbb{G}^{d}$ so that (4.31) holds with $g_{N}$ replaced by $v_{N}$. By Remark 7.20. of [8],

$$
\begin{aligned}
& \inf _{v \in \mathbb{G}^{d}} \sup _{\rho \in[0,1]}\left|\frac{1}{2} \widetilde{\mathbb{W}}_{k, j}^{v}(\rho)-\chi(\rho) D_{k, j}(\rho)\right| \\
& \leqslant \lim _{n \rightarrow \infty} \sup _{\rho \in[0,1]}\left|V_{\rho}\left(\mathbf{J}_{0, e_{j}}^{0}+\mathcal{L}^{0} v_{j, N}+\sum_{\ell=1}^{d} D_{j, \ell}(\rho) \frac{\psi_{n, n}^{e_{\ell}}}{n}, \mathbf{J}_{0, e_{k}}^{0}+\mathcal{L}^{0} v_{k, N}+\sum_{\ell=1}^{d} D_{k, \ell}(\rho) \frac{\psi_{n, n}^{e_{\ell}}}{n}\right)\right| \\
& \quad+\lim _{n \rightarrow \infty} \sup _{\rho \in[0,1]}\left|V_{\rho}\left(\sum_{\ell=1}^{\ell} D_{j, \ell}(\rho) \frac{\psi_{n, n}^{e_{\ell}}}{n}, \mathbf{J}_{0, e_{k}}^{0}+\mathcal{L}^{0} v_{k, N}+\sum_{\ell=1}^{d} D_{k, \ell}(\rho) \frac{\psi_{n, n}^{e_{\ell}}}{n}\right)\right| .
\end{aligned}
$$

By Schwartz inequality the right-hand side of (6.11) is bounded by

$$
\begin{aligned}
& \underset{n \uparrow \infty}{\limsup } \sup _{0 \leqslant \rho \leqslant 1}\left\{V_{\rho}^{1 / 2}\left(\mathbf{J}_{0, e_{j}}^{0}+\mathcal{L}^{0} v_{j, N}+\sum_{\ell=1}^{d} D_{j, \ell}(\rho) \frac{\psi_{n, n}^{e_{\ell}}}{n}\right) V_{\rho}^{1 / 2}\left(\mathbf{J}_{0, e_{k}}^{0}+\mathcal{L}^{0} v_{k, N}+\sum_{\ell=1}^{d} D_{k, \ell}(\rho) \frac{\psi_{n, n}^{e_{\ell}}}{n}\right)\right\} \\
& \quad+C \limsup _{n \uparrow \infty} \sup _{0 \leqslant \rho \leqslant 1} V_{\rho}^{1 / 2}\left(\mathbf{J}_{0, e_{k}}^{0}+\mathcal{L}^{0} v_{k, N}+\sum_{\ell=1}^{d} D_{k, \ell}(\rho) \frac{\psi_{n, n}^{e_{\ell}}}{n}\right)
\end{aligned}
$$

which is bounded by $\left\{\frac{1}{N}+C \frac{1}{\sqrt{N}}\right\}$ for some positive constant $C$. Letting $N \uparrow \infty$ the lemma is proved.

It is immediate to show the following.

Lemma 6.3. Let $\mu(\cdot, \cdot) \in \mathcal{D}^{0}$ with associate profile $\rho(\cdot, \cdot)$, then

$$
\mathcal{I}_{\text {lower }}(\mu(\cdot, \cdot))=\mathcal{I}_{\text {dyn }}(\mu(\cdot, \cdot)) \text {. }
$$

Proof. We have that

$$
\begin{array}{r}
\frac{1}{8} \int_{0}^{T} \mathrm{~d} t \int_{\Lambda}((\nabla V)(t, r) \cdot \sigma(\rho(t, r))(\nabla V)(t, r)) \mathrm{d} r=\sup _{\phi}\left\{\frac{1}{2} \int_{0}^{T} \mathrm{~d} t \int_{\Lambda}((\nabla V)(t, r) \cdot \sigma(\rho(t, r))(\nabla \phi)(t, r)) \mathrm{d} r\right. \\
-\frac{1}{2} \int_{0}^{T} \mathrm{~d} t \int_{\Lambda}((\nabla \phi)(t, r) \cdot \sigma(\rho(t, r))(\nabla \phi)(t, r) \mathrm{d} r\}
\end{array}
$$

where the supremum is taken over $\phi \in C^{1,2}([0, T] \times \Lambda)$. Since $\rho(\cdot, \cdot)$ is a weak solution of (4.6), for all $\phi \in$ $C^{1,2}([0, T] \times \Lambda)$ we have 


$$
\begin{aligned}
& \frac{1}{2} \int_{0}^{T} \mathrm{~d} t \int_{\Lambda}((\nabla V)(t, r) \cdot \sigma(\rho(t, r))(\nabla \phi)(t, r)) \mathrm{d} r \\
& =\int_{0}^{T} \int_{\Lambda} \partial_{t} \rho(t, r) \phi(t, r) \mathrm{d} r \mathrm{~d} t+\iint_{0}^{T}((\nabla \phi)(t, r) \cdot D(\rho(t, r))(\nabla \rho)(t, r)) \mathrm{d} r \mathrm{~d} t \\
& \quad-\frac{\beta}{2} \int_{0}^{T}((\nabla \phi)(t, r) \cdot \sigma(\rho(t, r))(\nabla(J \star \rho))(t, r)) \mathrm{d} r \mathrm{~d} t .
\end{aligned}
$$

Inserting this last identity in (6.13) we obtain the result.

\section{Extension of the lower bound}

To complete the proof of the lower bound it remains to show that for any $\mu(\cdot, \cdot) \in D\left([0, T], \mathcal{M}_{1}^{0}(\Lambda)\right)$, with $\mathcal{I}_{\text {dyn }}(\mu(\cdot, \cdot))$ finite we can find a sequence of $\mu^{\epsilon}(\cdot, \cdot) \in \mathcal{D}^{0}$ such that $\mu^{\epsilon}(\cdot, \cdot) \rightarrow \mu(\cdot, \cdot)$ in $D\left([0, T], \mathcal{M}_{1}(\Lambda)\right)$ and $\lim _{\epsilon \rightarrow 0} \mathcal{I}_{\text {dyn }}\left(\mu^{\epsilon}(\cdot, \cdot)\right)=\mathcal{I}_{\text {dyn }}(\mu(\cdot, \cdot))$. We define the class of profiles $\mathcal{E}$.

Definition of $\mathcal{E}$. We denote by $\mathcal{E} \subset D\left([0, T], \mathcal{M}_{1}^{0}(\Lambda)\right)$ the class of profiles $\rho(\cdot, \cdot)$ having $\mathcal{I}_{\text {dyn }}(\mu(\cdot, \cdot))$ finite.

Definition of $\mathcal{E}_{0}$. We denote by $\mathcal{E}_{0} \subset D\left([0, T], \mathcal{M}_{1}^{0}(\Lambda)\right)$ the class of evolving profiles $\rho(\cdot, \cdot)$ that are weak solutions of

$$
\frac{\partial \rho}{\partial t}=\frac{1}{2} \nabla \cdot\left(\sigma(\rho) \nabla\left\{\frac{\delta \mathcal{G}}{\delta \rho}-V(t, \cdot)\right\}\right)
$$

with some initial profile $0<\rho(0, \cdot)=\rho_{0}(\cdot)<1$, for some $V \in C^{1,2}([0, T] \times \Lambda)$. Further they have the following properties:

$$
\begin{aligned}
& \inf _{r \in \Lambda} \rho(t, r)>0 \text { for } t \in[0, T], \\
& \inf _{r \in \Lambda}(1-\rho(t, r))>0 \text { for } t \in[0, T] .
\end{aligned}
$$

Remark that $\mathcal{E}_{0} \subset \mathcal{D}^{0}$, see the beginning of Section 6 for the definition of $\mathcal{D}^{0}$. We have the following result.

Theorem 7.1. The $\mathcal{E}_{0}$ is properly dense in $\mathcal{E}$. That is, for any profile $\rho \in \mathcal{E}$ there exists a sequence $\left(\rho_{\epsilon}\right) \subset \mathcal{E}_{0}$, so that

$$
\lim _{\epsilon \rightarrow 0} \rho_{\epsilon}=\rho \quad \text { in the topology of } D\left([0, T], \mathcal{M}_{1}(\Lambda)\right)
$$

and

$$
\lim _{\epsilon \rightarrow 0} \mathcal{I}_{\text {dyn }}\left(\rho_{\epsilon}(\cdot, \cdot)\right)=\mathcal{I}_{\text {dyn }}(\rho(\cdot, \cdot))
$$

Proof. Denote $u(s, \cdot)$ for $s \in[0,1]$ the solution of

$$
\begin{aligned}
& \frac{\partial u}{\partial s}=\frac{1}{2} \nabla \cdot\left(\sigma(u) \nabla\left\{\frac{\delta \mathcal{G}}{\delta u}\right\}\right), \quad s \in(0,1), \\
& u(0, \cdot)=\rho(T, \cdot) .
\end{aligned}
$$

For each $s \in[0,1]$ we extend the definition of $\rho$ to $[T, T+1]$ setting $\rho(T+s, r)=u(s, r)$ where $u(\cdot, \cdot)$ is the solution of (7.4). For $s \in[0,1]$ denote by $\theta_{s} \rho$ the time translation of $\rho,\left(\theta_{s} \rho\right)(t, r)=\rho(t+s, r)$ for $(t, r) \in[0, T] \times \Lambda$. Since $u$ solves (7.4)

$$
\mathcal{I}_{\text {dyn }}\left(\left(\theta_{s} \rho\right)(\cdot, \cdot)\right) \leqslant \mathcal{I}_{\text {dyn }}(\rho(\cdot, \cdot)), \quad s \in[0,1] .
$$


Let $\Phi_{h}(\cdot)$ be the heat kernel on $\Lambda$ with periodic boundary conditions at time $1 / h^{2}$, which we use as mollifier. Define

$$
\rho_{h}(t, \cdot)=\left(\Phi_{h} \star \rho\right)(t, \cdot), \quad t \in[0, T] .
$$

Since the properties of the heat kernel $\rho_{h}(t, \cdot)>0,1-\rho_{h}(t, \cdot)>0$ for $t \in[0, T]$. For $\epsilon_{0}=2^{-n}$ consider the $C^{\infty}(\mathbb{R})$ mollifier $\Psi_{\epsilon_{0}}(s)$ for $s \in \mathbb{R}$, having support on $\left[0, \epsilon_{0}\right], \int \Psi_{\epsilon_{0}}(s) \mathrm{d} s=1$. Further set $\epsilon \equiv\left(\epsilon_{0}, h\right)$ and define

$$
\rho_{\epsilon}(t, r)=\int_{\mathbb{R}} \mathrm{d} s \Psi_{\epsilon_{0}}(s) \int_{\Lambda} \mathrm{d} y \Phi_{h}(r-y)\left(\theta_{s} \rho\right)(t, y), \quad t \in[0, T], r \in \Lambda .
$$

In the formula (7.7) we first take $\rho$, then we extend it and consider for any $s \in[0,1]$ the family of translated. We apply to each of them the smoothing in space $\Phi_{h}$, then we convolve with the convolution in time $\Psi_{\epsilon_{0}}$. We denote the result of these operations shortly by $\rho_{\epsilon}(t, r)$, for $(t, r) \in[0, T] \times \Lambda$, and $\epsilon \rightarrow 0$ stands for $h \uparrow \infty$ and $\epsilon_{0} \rightarrow 0$. Clearly $\rho_{\epsilon}(\cdot, \cdot) \in \mathcal{E}_{0}, \rho_{\epsilon} \in C^{\infty, \infty}([0, T] \times \Lambda), \rho_{\epsilon}(t, \cdot)>0,1-\rho_{\epsilon}(t, \cdot)>0$ and we can find for each $\epsilon$ an unique $V_{\epsilon} \in C^{1,2}([0, T] \times \Lambda)$ solution of Eq. (7.1) with initial condition $\left(\rho_{0}\right)_{\epsilon}$. Namely considering $t$ as a parameter we can solve for each fixed $t \in(0, T)$ and for each $\epsilon$

$$
\frac{1}{2} \nabla \cdot\left(\sigma\left(\rho_{\epsilon}(t)\right) \nabla\left\{V_{\epsilon}(t, \cdot)\right\}\right)=\frac{1}{2} \nabla \cdot\left(\sigma\left(\rho_{\epsilon}(t)\right) \nabla\left\{\frac{\delta \mathcal{G}}{\delta \rho_{\epsilon}}\left(\rho_{\epsilon}(t)\right)\right\}\right)-\frac{\partial \rho_{\epsilon}}{\partial t} .
$$

The (7.8) is an uniformly elliptic equation in $\Lambda$, having $\sigma\left(\rho_{\epsilon}(t, \cdot)\right)$ strictly positive and since by assumption $D(\cdot) \in$ $C^{1, a}([0,1])$ the solution $V(t, \cdot) \in C^{2}(\Lambda), t \in(0, T)$, see [19]. We define by continuity $V_{\epsilon}(t, \cdot)$ in 0 and $T$. Note that

$$
\frac{\partial \rho_{\epsilon}}{\partial t}(t, r)=\int_{\mathbb{R}} \mathrm{d} s \Psi_{\epsilon_{0}}(s)\left(\left(\theta_{s} \frac{\partial \rho}{\partial t}\right)\right)_{h}(t, r) \quad \text { for } t \in(0, T), r \in \Lambda .
$$

By construction $\lim _{\epsilon \rightarrow \infty} \rho_{\epsilon}=\rho$ in $D\left([0, T], \mathcal{M}_{1}(\Lambda)\right)$. Since $\mathcal{I}_{\text {dyn }}(\cdot)$ is lower semicontinuous it is enough to show that

$$
\limsup _{\varepsilon \rightarrow 0} \mathcal{I}_{\text {dyn }}\left(\rho_{\epsilon}(\cdot, \cdot)\right) \leqslant \mathcal{I}_{\text {dyn }}(\rho(\cdot, \cdot)) .
$$

The proof of (7.10) is handled in the same way as Lemma 6.8 of [26]. The finiteness of $\mathcal{I}_{\text {dyn }}(\rho(\cdot, \cdot))$ implies in particular that, see (3.8) and [27], there exists a vector $P(t, \cdot)$ so that $\partial_{t} \rho=\nabla \cdot P$ and

$$
\int_{0}^{T}\left\|\partial_{t} \rho\right\|_{-1, \sigma(\rho(t, \cdot))}^{2} \mathrm{~d} t=\iint_{0}^{T} \int_{\Lambda}\left(P(t, r) \cdot[\sigma(\rho(t, r))]^{-1} P(t, r)\right) \mathrm{d} r \mathrm{~d} t \leqslant C .
$$

From the definition

$$
\begin{aligned}
\mathcal{I}_{\text {dyn }}(\rho(\cdot, \cdot))= & \frac{1}{2} \iint_{0}^{T}\left(P(t, r) \cdot[\sigma(\rho(t, r))]^{-1} P(t, r)\right) \mathrm{d} t \mathrm{~d} r+\frac{1}{4} \mathcal{I}_{0}(\rho) \\
& -\frac{1}{2} \iint_{0}^{T}\left(P(t, r) \cdot \chi(\rho(t, r))^{-1} \nabla \rho(t, r)\right) \mathrm{d} t \mathrm{~d} r+\frac{\beta}{2} \int_{0}^{T} \int_{\Lambda}(P(t, r) \cdot \nabla(J \star \rho)(t, r)) \mathrm{d} t \mathrm{~d} r \\
& -\frac{\beta}{2} \int_{0}^{T} \int_{\Lambda} D(\rho(t, r)) \nabla \rho(t, r) \cdot \nabla(J \star \rho)(t, r) \mathrm{d} t \mathrm{~d} r \\
& +\frac{\beta^{2}}{8} \int_{0}^{T} \int_{\Lambda} \sigma(\rho(t, r)) \nabla(J \star \rho)(t, r) \cdot \nabla(J \star \rho)(t, r) \mathrm{d} t \mathrm{~d} r .
\end{aligned}
$$

Applying the inequality $a b \leqslant \frac{1}{2}\left[\lambda a^{2}+\frac{1}{\lambda} b^{2}\right]$ for $\lambda>0$ we obtain pointwise 


$$
\begin{aligned}
& \left(P \cdot \chi(\rho)^{-1} \nabla \rho\right)=\left(P \cdot \sigma(\rho)^{-1} \sigma(\rho) \chi(\rho)^{-1} \nabla \rho\right) \\
& \quad \leqslant \frac{1}{2} \lambda\left(P \cdot \sigma(\rho)^{-1} P\right)+\frac{1}{2 \lambda}\left(\chi(\rho)^{-1} \nabla \rho \cdot \sigma(\rho) \chi(\rho)^{-1} \nabla \rho\right) .
\end{aligned}
$$

Take $\lambda=2$ in (7.13), recall that $D=\frac{1}{2} \frac{\sigma}{\chi}$ and we obtain that

$$
\iint_{0}^{T}\left(P(t, r) \cdot \chi(\rho(t, r))^{-1} \nabla \rho(t, r)\right) \mathrm{d} r \mathrm{~d} t \leqslant \iint_{0}^{T}\left(P(t, r) \cdot \sigma(\rho(t, r))^{-1} P(t, r)\right) \mathrm{d} r \mathrm{~d} t+\frac{1}{2} \mathcal{I}_{0}(\rho) .
$$

Similarly we obtain that

$$
(P \cdot \nabla(J \star \rho)) \leqslant\left(P \cdot \sigma(\rho)^{-1} P\right)+\frac{1}{4}(\sigma \nabla(J \star \rho) \cdot \nabla(J \star \rho)) .
$$

Then since (7.11) and by assumption $\mathcal{I}_{0}(\rho)$ is finite, each single term of (7.12) is finite on its own. Therefore to obtain (7.10) it is sufficient to show that (7.11) implies the uniform integrability of

$$
\iint_{0}^{T}\left(P_{\epsilon}(t, r) \cdot \sigma\left(\rho_{\epsilon}(t, r)\right)^{-1} P_{\epsilon}(t, r)\right) \mathrm{d} r \mathrm{~d} t
$$

and to show that

$$
\lim _{\epsilon \rightarrow 0} \mathcal{I}_{0}\left(\rho_{\epsilon}(\cdot, \cdot)\right)=\mathcal{I}_{0}(\rho(\cdot, \cdot)) \text {. }
$$

Namely all the remaining terms in $\mathcal{I}_{\text {dyn }}\left(\rho_{\epsilon}(\cdot, \cdot)\right)$ converge to the respective terms in (7.12), since the continuity assumptions on $D(\cdot), \sigma(\cdot)$. The (7.15) is proved in Proposition 7.2, stated below. Next we show the uniform integrability of (7.14). Since (2.23) we have that pointwise

$$
\frac{|P|^{2}}{C \rho(1-\rho)} \leqslant\left(P \cdot \sigma(\rho)^{-1} P\right) \leqslant C \frac{|P|^{2}}{\rho(1-\rho)}
$$

where $|P|^{2}=\sum_{i=1}^{d}\left|P_{i}\right|^{2}$. Then we obtain

$$
\left(P_{\epsilon} \cdot \sigma\left(\rho_{\epsilon}\right)^{-1} P_{\epsilon}\right) \leqslant C \frac{\left|P_{\epsilon}\right|^{2}}{\rho_{\epsilon}\left(1-\rho_{\epsilon}\right)} .
$$

Further for a function $f(t, x)$

$$
\begin{aligned}
\left(f_{\epsilon}(t, x)\right)^{2} & =\left(\int_{\Lambda \times \mathbb{R}} \mathrm{d} s \mathrm{~d} y \Psi_{\epsilon_{0}}(s) \Phi_{h}(x-y)\left(\theta_{s} f\right)(t, y)\right)^{2} \\
& =\left(\int_{\Lambda \times \mathbb{R}} \mathrm{d} s \mathrm{~d} y \Psi_{\epsilon_{0}}(s) \Phi_{h}(x-y) \frac{\sqrt{\left(\theta_{s} \rho\right)(t, y)\left(1-\left(\theta_{s} \rho\right)(t, y)\right)}}{\sqrt{\left(\theta_{s} \rho\right)(t, y)\left(1-\left(\theta_{s} \rho\right)(t, y)\right)}}\left(\theta_{s} f\right)(t, y)\right)^{2} \\
& \leqslant\left(\frac{1}{\rho(1-\rho)} f^{2}\right)_{\epsilon}(t, x)(\rho(1-\rho))_{\epsilon}(t, x) .
\end{aligned}
$$

By convexity

$$
(\rho(1-\rho))_{\epsilon}(t, x) \leqslant \rho_{\epsilon}(t, x)\left(1-\rho_{\epsilon}(t, x)\right) .
$$

Then taking in account (7.17)-(7.19) and (2.23) we obtain pointwise that

$$
\left(P_{\epsilon} \cdot \sigma\left(\rho_{\epsilon}\right)^{-1} P_{\epsilon}\right) \leqslant C\left(\frac{|P|^{2}}{\rho(1-\rho)}\right)_{\epsilon} \leqslant C\left(\left(P \cdot[\sigma(\rho)]^{-1} P\right)\right)_{\epsilon} .
$$

The last term is uniformly integrable, which implies the uniformly integrability of $\left(P_{\epsilon} \cdot \sigma\left(\rho_{\epsilon}\right)^{-1} P_{\epsilon}\right)$. 
Proposition 7.2. Suppose $\mathcal{I}_{0}(\rho(\cdot, \cdot))$ finite and let $\rho_{\epsilon}(\cdot, \cdot)$ be as in $(7.7)$ then

$$
\lim _{\epsilon \rightarrow 0} \mathcal{I}_{0}\left(\rho_{\epsilon}(\cdot, \cdot)\right)=\mathcal{I}_{0}(\rho(\cdot, \cdot)) .
$$

Proof. By the boundedness of the diffusion coefficient and (2.23) we have that

$$
\begin{aligned}
& \frac{1}{C} \int_{0}^{T} \int_{\Lambda} \frac{|\nabla \rho(t, r)|^{2}}{\rho(t, r)(1-\rho(t, r))} \mathrm{d} t \mathrm{~d} r \leqslant \mathcal{I}_{0}(\rho(\cdot, \cdot))=\int_{0}^{T} \int_{\Lambda}\left(\nabla \rho(t, r) \cdot \frac{D(\rho(t, r))}{\chi(\rho(t, r))} \nabla \rho(t, r)\right) \mathrm{d} t \mathrm{~d} r \\
& \quad \leqslant C \int_{0}^{T} \int_{\Lambda} \frac{|\nabla \rho(t, r)|^{2}}{\rho(t, r)(1-\rho(t, r))} \mathrm{d} t \mathrm{~d} r .
\end{aligned}
$$

Denote

$$
X_{\epsilon}(t, r) \equiv\left(\nabla \rho_{\epsilon}(t, r) \cdot \frac{D\left(\rho_{\epsilon}(t, r)\right)}{\chi\left(\rho_{\epsilon}(t, r)\right)} \nabla \rho_{\epsilon}(t, r)\right)
$$

and

$$
X(t, r) \equiv\left(\nabla \rho(t, r) \cdot \frac{D(\rho(t, r))}{\chi(\rho(t, r))} \nabla \rho(t, r)\right) .
$$

As in the previous theorem, to show (7.21) it will be enough to prove that $\lim _{\epsilon \rightarrow 0} X_{\epsilon}=X$ in measure (Lebesgue) in $[0, T] \times \Lambda$, and that $X_{\epsilon}$ is uniformly integrable in $[0, T] \times \Lambda$. These two properties imply (7.21). One can show, as in the previous theorem, that

$$
X_{\epsilon}(t, r) \leqslant C \frac{\left|\nabla \rho_{\epsilon}(t, r)\right|^{2}}{\rho_{\epsilon}(t, r)\left(1-\rho_{\epsilon}(t, r)\right)} \leqslant C\left(\frac{|\nabla \rho|^{2}}{\rho(1-\rho)}\right)_{\epsilon}(t, r) \leqslant C\left(\nabla \rho \cdot \frac{D(\rho)}{\chi(\rho)} \nabla \rho\right)_{\epsilon}(t, r) .
$$

Then $X_{\epsilon}$ is uniformly integrable.

\section{Appendix A. Non-gradient tools}

We recall some tools used in the non-gradient methods. We refer mainly to [8], see also [14], Section VII. Given $\alpha \in \Omega_{D}$, denote by $\mathcal{L}^{0}$ the pregenerator of the DLG process in infinite volume (cf. (2.15)),

$$
\left(\mathcal{L}^{0} f\right)(\eta)=\sum_{e \in \mathcal{E}} \sum_{x \in \mathbb{Z}^{d}} C^{0}(x, x+e ; \eta)\left[\left(\nabla_{x, x+e} f\right)(\eta)\right],
$$

where $f$ is a local function on $\mathcal{S}$. We refer to [16] for the construction of the process in the infinite volume setting, and we recall that for every $\lambda \in \mathbb{R}, \mathcal{L}^{0}$ can be extended to a self-adjoint operator on $L^{2}\left(\mu^{\alpha, \lambda}\right)$. For a finite non-empty subset $B$ of $\mathbb{Z}^{d}, \rho \in\left[0,|B|^{-1}, \ldots, 1\right]$ and $\alpha \in \Omega_{D}$, the canonical measure $\nu_{\rho, B}^{\alpha}$ is defined as in (2.17), with $\Lambda_{\gamma}$ replaced by $B$. We denote by $\mathcal{M}^{\alpha}(B)$ the set of all canonical measures as $\rho$ varies in $\left[0,|B|^{-1}, \ldots, 1\right]$, and by $v^{\alpha}$ a generic element of $\mathcal{M}^{\alpha}(B)$. Let $\mathcal{G} \subset \mathbb{G}$, see (2.18), be the space of bounded cylinder functions $h$ for which there exists a finite non-empty set $B \subset \mathbb{Z}^{d}$ so that the support of $h(\cdot, \alpha)$ is contained in $B$ and, for any disorder configuration $\alpha \in \Omega_{D}$, all canonical expectations on $B$ are null:

$$
\mathcal{G}=\left\{h \in \mathbb{G} ; \text { support of }\{h(\cdot, \alpha)\} \subset B \text { and } \forall \alpha \in \Omega_{D}, \forall \nu^{\alpha} \in \mathcal{M}^{\alpha}(B), \mathbf{E}^{\nu^{\alpha}}[h(\cdot, \alpha)]=0\right\} .
$$

Given a positive density $0<\rho<1, f$ and $g$ in $\mathcal{G}$, define

$$
V_{\rho}(h, g)=\lim _{\ell \rightarrow \infty}(2 \ell)^{-d} \mathbb{E} g\left[\mathbf{E}^{\mu^{\alpha, \lambda_{0}(\rho)}}\left(\sum_{|x| \leqslant \ell-\sqrt{\ell}} \tau_{x} h,\left(-\mathcal{L}_{\ell}^{0}\right)^{-1} \sum_{|x| \leqslant \ell-\sqrt{\ell}} \tau_{x} g\right) g\right],
$$

where $\mathcal{L}_{\ell}^{0}$ is obtained from $\mathcal{L}^{0}$ by restricting jumps to $\Lambda_{0, \ell}$, the cube centered at the origin of side $\ell$ and $\lambda_{0}(\rho)$ is the annealed chemical potential corresponding to the particle density $\rho$, see (2.6). In the extreme densities cases $\rho=0$ or $\rho=1$, i.e. when the measure is concentrated on configurations $\eta=0$ or $\eta=1$ in $\Lambda_{0, \ell}$, for any $\ell \in \mathbb{Z}$, set $V_{\rho}(h, g)=0$. 
It has been shown in [8], Theorem 7.2, that the above limit exists and is finite. Moreover $V_{\rho}(\cdot, \cdot)$ defines a semi-inner product on $\mathcal{G}$. When $h=g$ we write $V_{\rho}(h)$ in place of $V_{\rho}(h, h)$. Given $s=2 \ell+1$ with $\ell \in \mathbb{N}$ and $e \in \mathcal{E}$, let $\Lambda_{1, s}^{e}$ and $\Lambda_{2, s}^{e}$ be a couple of adjacent cubes of diameter $s$, centered respectively at $-(\ell+1) e$ and at $\ell e$. For any configuration $\eta$, denote by $m_{s}^{1, e}, m_{s}^{2, e}$, and $m_{s}^{e}$ the densities respectively in $\Lambda_{1, s}^{e}, \Lambda_{2, s}^{e}$, and $\Lambda_{2, s}^{e} \cup \Lambda_{1, s}^{e}$. Given an integer $s^{\prime}$ with $s \leqslant s^{\prime}$, set

$$
\phi_{s, s^{\prime}}^{e}=\mathbf{E}^{\mu^{\alpha}}\left[m_{s}^{2, e}-m_{s}^{1, e} \mid m_{s^{\prime}}^{e}\right] \quad \text { and } \quad \psi_{s, s^{\prime}}^{e}=m_{s}^{2, e}-m_{s}^{1, e}-\phi_{s, s^{\prime}}^{e} .
$$

Note that $\mathbb{E}\left[\phi_{s, s^{\prime}}^{e}\right]=0$. The main step to obtain a generalized Fick's law, see [8] Proposition 7.18, is to show the following property:

(P) For $d \geqslant 3$ and for any $e \in \mathcal{E},\left(\left(\psi_{n, n}^{e}\right) / n\right)_{n} \geqslant 0$ is a Cauchy sequence in the space $\mathcal{G}$ endowed with the semi-inner product $V_{\rho}$, and its limit points $\left(\psi_{e}\right)_{e \in \mathcal{E}}$ form a basis of the subspace $\left(\mathcal{L}^{0} \mathbb{G}\right)^{\perp}$.

An important step to prove this, see [8] Section 7.2, is the introduction of the following auxiliary functions. For the integer $s=2 \ell+1, \ell \in \mathbb{N}$ and $e \in \mathcal{E}$, let

$$
\mathbf{W}_{s}^{e}=\frac{1}{\left|\Lambda_{1, s}^{e}\right|} \sum_{x \in \Lambda_{1, s}^{e}}\left\{\frac{1}{\left|\Lambda_{2, s}^{e}\right|} \sum_{y \in \Lambda_{2, s}^{e}} \omega_{x, y}^{\alpha}\right\},
$$

where

$$
\omega_{x, y}^{\alpha}=\left(1+\mathrm{e}^{-(\alpha(x)-\alpha(y))(\eta(x)-\eta(y))}\right)(\eta(y)-\eta(x))
$$

and $\Lambda_{1, s}^{e}$ and $\Lambda_{2, s}^{e}$ are the cubes defined before (A.4). When $x$ and $y$ are nearest neighbors, $\omega_{x, y}^{\alpha}$ is the current associated to a particular choice of the rate $C^{0}(x, y ; \eta)$ corresponding to $\Phi(E)=1+\mathrm{e}^{-E}$ in (2.16). It has the important property to have mean zero with respect to any measure $v^{\alpha}$ in $\mathcal{M}^{\alpha}(B), B \subset \mathbb{Z}^{d}$ being any bounded set containing $x$ and $y$. Furthermore it yields a simple integration by parts formula

$$
\int \omega_{x, y}^{\alpha} f(\eta) \mathrm{d} \nu^{\alpha}(\eta)=\int[\eta(x)-\eta(y)]\left(\nabla_{x, y} f\right)(\eta) \mathrm{d} \nu^{\alpha}(\eta) .
$$

It is proved in [8], Theorem 7.11, that for any $e \in \mathcal{E}$ and $0 \leqslant \rho \leqslant 1$,

$$
\lim _{n \uparrow \infty} V_{\rho}\left(2 \rho(1-\rho) \lambda^{\prime}{ }_{0}(\rho) \frac{\psi_{n, n}^{e}}{n}-\frac{\mathbf{W}_{n}^{e}}{n}\right)=0 .
$$

Moreover, if for $g \in \mathbb{G}$ and $h \in \mathcal{G}$ we define

$$
(h, g)_{\rho, 0}=\sum_{x \in \mathbb{Z}^{d}} \mathbb{E}\left[\mathbf{E}^{\mu^{\alpha, \lambda_{0}(\rho)}}\left(h, \tau_{x} g\right)\right],
$$

we obtain by the definition of $V_{\rho}(\cdot, \cdot)$ the following properties (cf. Lemma 7.1 of [8]):

$$
\begin{aligned}
& V_{\rho}\left(h, \mathcal{L}^{0} g\right)=-(h, g)_{\rho, 0}, \\
& V_{\rho}\left(\mathbf{J}_{0, e_{k}}^{0}, \mathbf{J}_{0, e_{m}}^{0}\right)=\frac{\delta_{k, m}}{2} \mathbb{E}\left[\mathbf{E}^{\mu^{\alpha, \lambda_{0}(\rho)}}\left(C^{0}\left(0, e_{k} ; \eta\right)\left(\nabla_{0, e_{k}} \eta(0)\right)^{2}\right)\right], \\
& V_{\rho}\left(\mathbf{J}_{0, e_{k}}^{0}, \frac{\mathbf{W}_{n}^{e_{m}}}{n}\right)=-\delta_{k, m} 2 \rho(1-\rho),
\end{aligned}
$$

where $\delta_{k, m}$ is the Kroenecker delta and $\mathbf{J}_{0, e_{k}}^{0}$ is defined in (4.10). Thanks to (A.5) and to the last identity in (A.7) one obtains, cf. (7.16) of [8], that

$$
\lim _{n \rightarrow \infty} V_{\rho}\left(\mathbf{J}_{0, e_{k}}^{0}, \frac{\psi_{n, n}^{e_{m}}}{n}\right)=V_{\rho}\left(\mathbf{J}_{0, e_{k}}^{0}, \psi_{e_{m}}\right)=-\delta_{k, m} \chi(\rho)
$$




\section{Acknowledgements}

The authors are grateful to Olivier Benois for useful discussions and to Abdellatif Koukkous, who was present in the earlier part of the work. Mustapha Mourragui thanks Claudio Landim for suggestions and the Department of Mathematics of the University of Roma TRE. Enza Orlandi thanks Lorenzo Bertini for helpful discussions, Alessandra Faggionato for pointing out a reference and the Department of Mathematics of Rouen University, where part of the work has been accomplished, for warm hospitality.

\section{References}

[1] M. Aizenman, J. Wehr, Rounding of first order phase transitions in systems with quenched disorder, Comm. Math. Phys. 130 (1990) $489-528$.

[2] A. Asselah, G. Giacomin, Metastability for the exclusion process with mean-field interaction, J. Stat. Phys. 93 (5/6) (1998) 1051-1110.

[3] L. Bertini, Dynamic fluctuations for Kac and related models, Markov Process. Related Fields 8 (2) (2002) 365-379.

[4] J. Bricmont, A. Kupiainen, Phase transition in the three-dimensional random field Ising model, Comm. Math. Phys. 116 (1988) 539-572.

[5] M. Cassandro, E. Orlandi, P. Picco, Typical configurations for one-dimensional random field Kac model, Ann. Probab. 27 (3) (1999) 14141467.

[6] M. Cassandro, E. Orlandi, P. Picco, M.E. Vares, Typical configurations for one-dimensional random field Kac model: localization of the phases, Electronic J. Probab. 10 (2005) 786-864.

[7] A. De Masi, E. Orlandi, L. Triolo, E. Presutti, Glauber evolution with Kac potentials. I. Mesoscopic and macroscopic limits, interface dynamics, Nonlinearity 7 (1996) 287-301;

A. De Masi, E. Orlandi, L. Triolo, E. Presutti, Glauber evolution with Kac potentials. II. Fluctuation, Nonlinearity 9 (1996) 27-51;

A. De Masi, E. Orlandi, L. Triolo, E. Presutti, Glauber evolution with Kac potentials. III. Spinodal decomposition, Nonlinearity 9 (1996) 53-114.

[8] A. Faggionato, F. Martinelli, Hydrodynamic limit of a disordered lattice gas, Probab. Theory Related Fields 127 (4) (2003) $535-608$.

[9] G. Giacomin, J.L. Lebowitz, Phase segregation dynamics in particle systems with long range interaction. I. Macroscopic limits, J. Stat. Phys. 87 (1997) 37-61;

G. Giacomin, J.L. Lebowitz, Phase segregation dynamics in particle systems with long range interaction. II. Interface motion, SIAM J. Appl. Math. 58 (1998) 1707-1729.

[10] G. Giacomin, J.L. Lebowitz, R. Marra, Macroscopic evolution of particle systems with short- and long-range interactions, Nonlinearity 13 (2000) 2143-2162.

[11] G. Giacomin, J.L. Lebowitz, E. Presutti, Deterministic and stochastic hydrodynamic equations arising from simple microscopic model systems, in: Stochastic Partial Differential Equations: Six Perspectives, Math. Surveys Monogr., vol. 64, American Mathematical Society, Providence, RI, 1999.

[12] M. Kac, G. Uhlenbeck, P.C. Hemmer, On the van der Waals theory of vapour-liquid equilibrium. I. Discussion of a one-dimensional model, J. Math. Phys. 4 (1963) 216-228;

M. Kac, G. Uhlenbeck, P.C. Hemmer, On the van der Waals theory of vapour-liquid equilibrium. II. Discussion of the distribution functions, J. Math. Phys. 4 (1963) 229-247;

M. Kac, G. Uhlenbeck, P.C. Hemmer, On the van der Waals theory of vapour-liquid equilibrium. III. Discussion of the critical region, J. Math. Phys. 5 (1964) 60-74.

[13] K.W. Kehr, T. Wichmann, in: A. Pekalski (Ed.), Diffusion Processes: Experiment, Theory, Simulations, Lecture Notes in Physics, vol. 438, Springer, 1994.

[14] C. Kipnis, C. Landim, Hydrodynamic Limit of Interacting Particle Systems, Springer-Verlag, 1999.

[15] C. Kipnis, S. Olla, S.R.S. Varadhan, Hydrodynamic and large deviations for simple exclusion process, Comm. Pure Appl. Math. 42 (1989) 115-137.

[16] T. Liggett, Interacting Particles Systems, Springer, Berlin, 1985.

[17] J.L. Lebowitz, E. Orlandi, E. Presutti, A particle model for spinodal decomposition, J. Stat. Phys. 63 (1991) 933-974.

[18] J. Lebowitz, O. Penrose, Rigorous treatment of the Van der Waals Maxwell theory of the liquid-vapour transition, J. Math. Phys. 7 (1966) 98-113.

[19] O.A. Ladyzenskaja, N.N. Uralceva, Equations aux derivées partielles de type elliptique, Editions Nauka, Moscou, 1964.

[20] R. Marra, M. Mourragui, Phase segregation dynamics for the Blume-Capel model with Kac interaction, Stochastic Process. Appl. 88 (2000) 79-124.

[21] M. Mourragui, E. Orlandi, E. Saada, Macroscopic evolution of particles systems with random field Kac interactions, Nonlinearity 16 (2003) 2123-2147.

[22] T. Nattermann, Theory of the random field Ising model, in: P. Young (Ed.), Spin Glasses and Random Fields, World Scientific, 1997.

[23] E. Presutti, From Statistical Mechanics towards Continuum Mechanics, Notes of lectures held at the Max-Planck Institute, Leipzig, 1999.

[24] J. Quastel, Diffusion in disordered media, in: T. Funaki, W. Woyczinky (Eds.), Proceedings on Stochastic Method for Nonlinear PDE, IMA Volumes in Mathematics, vol. 77, Springer-Verlag, 1995, pp. 65-79.

[25] J. Quastel, Large deviations from a hydrodynamic scaling limit for a nongradient system, Ann. Probab. 23 (2) (1995) 724-742.

[26] J. Quastel, F. Rezakhanlou, S.R.S. Varadhan, Large deviations for the symmetric simple exclusion process in dimension $d \geqslant 3$, Probab. Theory Related Fields 113 (1999) 1-84. 
[27] J. Quastel, S.R.S. Varadhan, Diffusion semigroup and diffusion processes corresponding to degenerate divergence form operators, Comm. Pure Appl. Math. L (1997) 667-706.

[28] H. Spohn, Large Scale Dynamics of Interacting Particles, Springer, Berlin, 1991.

[29] S.R.S. Varadhan, Nonlinear diffusion limit for a system with nearest neighbor interactions. II, in: K. Elworthy, N. Ikeda (Eds.), Asymptotic Problems in Probability Theory: Stochastic Models and Diffusion on Fractals, Pitman Research Notes in Mathematics, vol. 283 , Wiley, 1994.

[30] S.R.S. Varadhan, H.T. Yau, Diffusive limit of lattice gas with mixing conditions, Asian J. Math. 1 (1997) 623-678. 